\title{
Motivic Sheaves and Filtrations on Chow Groups
}

\author{
UWE JANNSEN
}

Grothendieck's motives, as described in [Dem, Kl2, Ma] are designed as a tool to understand the cohomology of smooth projective varieties and the algebraic cycles modulo homological and numerical equivalence on them. According to Beilinson and Deligne, Grothendieck's category of pure motives should embed in a bigger category of mixed motives that allows the treatment of arbitrary varieties and an understanding of the whole Chow group of cycles modulo rational equivalence, in fact, even of all algebraic $K$-groups of the varieties.

In this paper we review some of these ideas and discuss some consequences. In particular, we show how the vast conjectural framework set up by Beilinson leads to very explicit conjectures on the existence of certain filtrations on Chow groups of smooth projective varieties. These filtrations would offer an understanding of several phenomena and counterexamples that for some time have led people to believe that the behaviour of the algebraic cycles is absolute chaos for codimension bigger than one.

In $\S 1$ we review some basic facts on Chow groups, correspondences, and cycle maps into cohomology theories. We recall a counterexample of Mumford implying that in general Chow groups are not representable and the Abel-Jacobi map has a huge kernel and some investigations of Bloch on this topic.

In $\S \S 2$ and 4 we state altogether four versions of Beilinson's conjectures on mixed motives and filtrations on Chow groups, increasing in generality and sophistication. The first one does not even mention mixed motives and proposes finite filtrations $F^{0} \supset F^{1} \supset \cdots$ on rational Chow groups $C H^{j}(X)_{\mathbb{Q}}$ that are uniquely determined by their behaviour under algebraic correspondences. The first step is homological equivalence, but the following steps differ very much from those considered classically. For example, algebraic equivalence does not appear, and the second step is something like the kernel

1991 Mathematics Subject Classification. Primary 14C15; Secondary 14A20, 14C25.

This paper is in final form and no version of it will be submitted for publication elsewhere. 
of (all) Abel-Jacobi maps. The crucial property is that the actions of correspondences on the graded pieces $\operatorname{Gr}_{F}^{\nu} C H^{j}(X)_{\mathbb{Q}}$ factor through homological equivalence. This would very nicely bridge the gap between homological and rational equivalence, in finitely many steps.

The described "filtration conjecture" can thus be regarded as a counterpart of the standard conjectures, being responsible for the part not covered by the latter. It is amazing to realize that the filtration conjecture would follow from the injectivity of suitable cycle maps, while (parts of) the standard conjectures would follow from the surjectivity of cycle maps, viz., the conjectures of Hodge and Tate.

While by definition $\operatorname{Gr}_{F}^{0} C H^{j}(X)_{\mathbb{Q}}$ is the space of algebraic cycles in the cohomology $H^{2 j}(X)$ of degree $2 j$, the higher graded pieces are related to $H^{2 j-1}(X), H^{2 j-2}(X)$, and so on. In fact, version 2 of the conjecture proposes the description (called "Beilinson's formula" in the following)

$$
\operatorname{Gr}_{F}^{\nu} C H^{j}(X)_{\mathbb{Q}}=\operatorname{Ext}_{\text {M }_{k}}^{\nu}\left(1, h^{2 j-\nu}(X)(j)\right),
$$

in terms of Yoneda extensions in the conjectural category $\mathscr{M}_{k}$ of mixed motives, where 1 is the trivial motive and $h^{i}(X)$ is the pure motive corresponding to the $i$ th cohomology of $X$. This is closely related to higher cycle maps, and indeed Mumford's counterexample and the above isomorphisms suggest studying higher than secondary (= Abel-Jacobi) maps to understand the whole Chow group.

Version 3 of the conjecture reveals more of the framework of mixed motives. Recall that $X \mapsto \bigoplus h^{i}(X)$ is the universal Weil cohomology theory for smooth projective varieties $X$ and that by definition an element $\alpha \in$ $C H^{j}(X)_{\mathbb{Q}}$ corresponds to a morphism $\varphi_{\alpha}: 1 \rightarrow h^{2 j}(X)(j)$ in Grothendieck's category $\mathscr{M}_{k}$ of pure motives. Roughly speaking, Beilinson's formula should come from a derived version: If one believes that $h^{\cdot}(X)$ arises as the cohomology of a complex $R(X)$ in $D^{b}\left(\mathscr{M}_{k}\right)$, the bounded derived category of $\mathscr{M} \mathscr{M}_{k}$, and the morphism $\varphi_{\alpha}$ from a morphism $\eta_{\alpha}: 1 \rightarrow R(X)(j)[2 j]$ (such that $\varphi_{\alpha}$ is obtained by passing to the 0th cohomology), then this leads to a cycle map

$$
C H^{j}(X)_{\mathbb{Q}} \rightarrow \operatorname{Hom}_{D^{b}\left(\mu_{\mathbb{N}_{k}}\right)}(1, R(X)(j)[2 j])
$$

and to an induced filtration via the spectral sequence

$$
\operatorname{Ext}_{{\mathscr{M K _ { k }}}_{k}}^{p}\left(1, h^{q}(X)(j)\right) \Rightarrow \operatorname{Hom}_{D^{b}\left(\mathscr{M K}_{k}\right)}(1, R(X)(j)[p+q]) .
$$

The optimistic conjecture says that (1) is an isomorphism. This together with the degeneration of (2) would give Beilinson's formula (0).

All this only reflects the situation encountered in the $\ell$-adic cohomology, and in fact, (2) is the motivic analogue of the Hochschild-Serre spectral sequence and (1) is the analogue of the cycle map into $\ell$-adic cohomology over $k$. The most general version 4 of Beilinson's conjecture expresses the hope 
that there is a theory of mixed motives that resembles and parallels closely the general theory of $\ell$-adic cohomology and $\ell$-adic sheaves. In particular, one hopes for relative and local versions of mixed motives, the so-called mixed motivic sheaves.

In $\S 3$ we state some remarkable consequences of Beilinson's conjectures which already follow from the down-to-earth version 1 , but which we derive via Beilinson's formula, to demonstate its use and usefulness. In particular, we show that the conjectures would lead to a good understanding of the representability of Chow groups. Guided by the conjectural theory, we prove some results on the nonrepresentability of Chow groups. This extends work of Mumford, Bloch, Roitman, and others and may be interesting in its own right.

Finally, in $\S 5$ we discuss the relationship with a conjectural filtration proposed by Murre. This proposal has the advantage of being quite explicit, in the formulation close to the standard conjectures, and more amenable to being proved in part. Whereas Murre arrived at this conjecture by the consideration of decompositions of motives, it was quickly clear to several experts that it is implied by Beilinson's conjectures. At the Seattle "motives" conference I discussed a partial converse, and soon after it occurred to me that Murre's conjecture is in fact equivalent to version 1 of Beilinson's conjecture.

This paper would not be complete without mentioning that Grothendieck certainly envisioned a much more general theory of motives than just for smooth projective varieties over a field. In particular, he already thought about motives over arbitrary bases and a general motivic duality formalism. This becomes quite clear in a letter written by Grothendieck to Illusie in 1973 which is reproduced in an appendix. This letter also addresses several interesting questions on motives that $I$ have not seen discussed elsewhere.

I am indebted to L. Illusie and W. Messing for providing me with the letter in the appendix and other "historical" material on motives, and to S. Kleiman for numerous helpful comments on this paper. The main result of $\S 5$ was obtained during a visit of Leiden University, which I thank for its hospitality. It is a pleasure to thank J. P. Murre and S. Saito for stimulating discussions. My stay at the Seattle conference was partially supported by the DFG, whose support is gratefully acknowledged.

\section{Higher cycle maps and Bloch's conjecture}

1.1. Let $X$ be a smooth projective variety over a field $k$. For any integer $j \geq 0$, the set $X^{(j)}=\{x \in X \mid \operatorname{codim} x=j\}$ of points of codimension $j$ can be identified with the set of closed irreducible subvarieties $Z$ of codimension $j$ in $X$ (by mapping $x$ to its closure $Z=\overline{\{x\}}$ and $Z$ to its generic point). Recall that the group of cycles of codimension $j$ on $X$ is the free abelian group $Z^{j}(X)=\bigoplus_{x \in X^{(j)}} \mathbb{Z}$ on $X^{(j)}$ and that the $j$ th Chow group $C H^{j}(X)$ is the quotient of $Z^{j}(X)$ modulo cycles that are rationally equivalent to zero 
[K12, §2]. By Quillen [Qui, §7, proof of 5.14], we can write this as

$$
C H^{j}(X)=\operatorname{Coker}\left(\bigoplus_{x \in X^{(j-1)}} k(x) \stackrel{\text { div }}{\rightarrow} \bigoplus_{x \in X^{(j)}} \mathbb{Z}\right),
$$

where $k(x)$ is the residue field of $x$ (= the function field of $Z=\overline{\{x\}}$ ) and div is the divisor map (cf. also [Fu, 1.3 and 1.6] for Chow groups that are graded by dimension).

The following operations on cycles are (only) well defined modulo rational equivalence. One has an intersection product

$$
\begin{aligned}
C H^{i}(X) \times C H^{j}(X) & \rightarrow C H^{i+j}(X), \\
(\alpha, \beta) & \mapsto \alpha \cdot \beta,
\end{aligned}
$$

making $C H^{*}(X)$ into a commutative ring, and for a morphism $f: X \rightarrow Y$ of smooth projective varieties one has pull-back maps

$$
f^{*}: C H^{j}(Y) \rightarrow C H^{j}(X), \quad j \geq 0,
$$

inducing a ring morphism $C H^{*}(Y) \rightarrow C H^{*}(X)$, and a push-forward map

$$
f_{*}: C H^{*}(X) \rightarrow C H^{*}(Y)
$$

mapping $C H^{d+i}(X)$ to $C H^{e+i}(Y)$, if $X$ and $Y$ are of pure dimensions $d$ and $e$, respectively. These operations enjoy the following compatibilities:

(a) (functoriality) $(g f)^{*}=f^{*} g^{*}$ and $(g f)_{*}=g_{*} f_{*}$ for a second morphism $g: Y \rightarrow Z$.

(b) (projection formula) $f_{*} \alpha \cdot \beta=f_{*}\left(\alpha \cdot f^{*} \beta\right)$.

(c) (base change) For a Cartesian diagram of projections

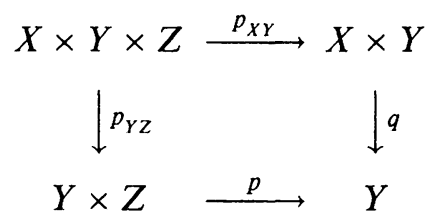

one has $p^{*} q_{*}=\left(p_{Y Z}\right)_{*} p_{X Y}^{*}$.

These properties ensure that one has a bilinear, associative composition law of correspondences ( $=$ algebraic cycles on products)

$$
\begin{aligned}
& C H^{e+s}(X \times Y \times Z) \times C H^{d+r}(X \times Y \times Z) \dot{\rightarrow} C H^{d+r+e+s}(X \times Y \times Z) \\
& \uparrow p_{Y Z}^{*} \quad \uparrow p_{X Y}^{*} \quad \downarrow\left(p_{X Z}\right) \text {. } \\
& C H^{e+s}(Y \times Z) \quad \times \quad C H^{d+r}(X \times Y) \rightarrow C H^{d+r+s}(X \times Z) \\
& (g, f) \mapsto g \circ f=\left(p_{X Z}\right)_{*}\left(p_{Y Z}^{*} g \cdot p_{X Y}^{*} f\right)
\end{aligned}
$$

for $X$ and $Y$ of pure dimensions $d$ and $e$, respectively, making the diagram commutative. In particular, $C H^{d}(X \times X)$ is an associative ring with unit 
(consider $X=Y=Z$; the unit is the class of the diagonal $\Delta: X \hookrightarrow X \times X)$, and one has an action of correspondences on Chow groups (consider $X=$ Spec $k)$ :

$$
\begin{aligned}
C H^{e+s}(Y \times Z) \times C H^{r}(Y) & \rightarrow C H^{r+s}(Z), \\
(\alpha, y) & \mapsto \alpha y=\left(p_{Z}\right)_{*}\left(\alpha \cdot p_{Y}^{*} y\right) .
\end{aligned}
$$

1.2. More generally, algebraic correspondences act on generalized cohomology theories. These are contravariant functors

$$
H: X \mapsto H^{*}(X, *)
$$

from the category $\mathscr{V}_{k}$ of smooth projective varieties over $k$ to the category of bi-graded $R$-algebras, for a ring $R$, equipped with the following additional structure:

(I) For every morphism $f: X \rightarrow Y$ in $\mathscr{V}_{k}$ there is a map of $R$-modules $f_{*}: H^{*}(X, *) \rightarrow H^{*}(Y, *)$, mapping $H^{2 d+i}(X, d+j)$ to $H^{2 e+i}(Y, e+j)$ if $X$ and $Y$ are of pure dimensions $d$ and $e$, respectively, such that the properties (a)-(c) hold correspondingly, where $f^{*}=H(f)$.

(II) There are cycle maps

$$
c l: C H^{j}(X) \rightarrow H^{2 j}(X, j)
$$

compatible with pull-back, push-forward, and products.

In fact, by (I) one has analogues of the diagram (1.2) and, hence, composition and action of "cohomological" correspondences, i.e., elements in $H^{*}(X \times Y, *)$. By (II), algebraic correspondences act via their cycle classes. Explicitly, we have the following diagram, for $X$ of pure dimension $d$,

$$
\begin{aligned}
& H^{2 d+2 r}(X \times Y, d+r) \times H^{i}(X \times Y, m) \rightarrow H^{2 d+i+2 r}(X \times Y, d+m+r) \\
& C H^{d+r}(X \times Y) \quad \times \quad H^{i}(X, m) \quad \rightarrow \quad H^{i+2 r}(Y, m+r) .
\end{aligned}
$$

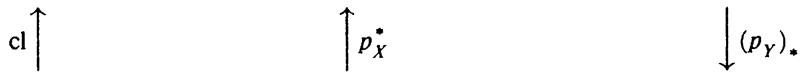

1.3. Examples of generalized cohomology theories are:

(1) The Chow theory:

$$
H^{i}(X, j)= \begin{cases}C H^{j}(X), & i=2 j, \\ 0 & \text { otherwise },\end{cases}
$$

where $R=\mathbb{Z}$ and $c l$ is the identity.

(2) Every Weil cohomology theory $X \mapsto H^{*}(X)$ (cf. [K13, Chapter 3])

$$
H^{i}(X, j)=H^{i}(X) \text { for all } j .
$$

(3) Singular cohomology with Hodge-Tate twists

$$
H^{i}(X, j)=H^{i}(X(\mathbb{C}), \mathbb{Z}(j)), \quad \mathbb{Z}(j)=\mathbb{Z}(2 \pi \sqrt{-1})^{j},
$$

for $k=\mathbb{C}$, where $R=\mathbb{Z}$ and the cycle class is renormalized (cf. the discussion in $[\mathrm{Ja} 3, \S 5])$; 
(4) $\ell$-adic cohomology with Tate twists

$$
H^{i}(X, j)=H_{\text {ét }}^{i}\left(\bar{X}, \mathbb{Z}_{\ell}(j)\right), \quad \bar{X}=X \times_{k} \bar{k},
$$

for $\ell \neq \operatorname{char}(k)$, where $R=\mathbb{Z}_{\ell}$. Here $\bar{k}$ is a separable closure of $k$ and $\mathbb{Z}_{\ell}(j)=\mathbb{Z}_{\ell}(1)^{\otimes j}$, where $\mathbb{Z}_{\ell}(1)=\lim _{n} \mu_{\ell^{n}}$ for the sheaves $\mu_{\ell^{n}}$ of $\ell^{n}$ th roots of unity.

(5) Deligne cohomology [Be1; EV, §1]

$$
H^{i}(X, j)=H_{\mathscr{D}}^{i}(X, \mathbb{Z}(j))
$$

for $k=\mathbb{C}$.

(6) $\ell$-adic cohomology over $k$

$$
H^{i}(X, j)=H_{\text {ét }}^{i}\left(X, \mathbb{Z}_{\ell}(j)\right)
$$

(naive or continuous $[\mathbf{J a 1}]$ ) for $\ell \neq \operatorname{char}(k)$.

After tensoring with $\mathbb{Q}$, examples (3) and (4) are also examples (the principal ones) of Weil cohomology theories, but we have some additional structure: In example (3) the cohomology group $H^{i}(X, j)$ carries a pure Hodge structure of weight $i-2 j$, by identifying it with the tensor product of $H^{i}(X(\mathbb{C}), \mathbb{Z})$, which has the usual Hodge structure of weight $i$, and the Hodge structure $\mathbb{Z}(j)$, which has weight $-2 j$ (cf. [De2, 2.1 and 2.2]). Then cup-product, pull-backs, and push-forwards are compatible with the Hodge structures, and the fact that the cycle map has image in

$$
H^{2 j}(X(\mathbb{C}), \mathbb{Z}(j)) \cap H^{j, j}(X)
$$

implies that the action of correspondences respects the Hodge structures. The last statement becomes quite obvious, if one identifies the above group with

$$
\operatorname{Hom}_{\mathrm{HS}}\left(\mathbb{Z}, H^{2 j}(X(\mathbb{C}), \mathbb{Z}(j))\right),
$$

the group of homomorphisms of Hodge structures, where $\mathbb{Z}$ is the trivial Hodge structure. In example (4), the absolute Galois group $G_{k}=\operatorname{Gal}(\bar{k} / k)$ of $k$ acts continuously on $H^{i}(X, j)$ by functoriality of étale cohomology. Since the cycle map has image in the fixed module

$$
H^{2 j}\left(\bar{X}, \mathbb{Z}_{\ell}(j)\right)^{G_{k}} \cong \operatorname{Hom}_{G_{k}}\left(\mathbb{Z}_{\ell}, H^{2 j}\left(\bar{X}, \mathbb{Z}_{\ell}(j)\right)\right)
$$

the action of correspondences respects the Galois actions. We remark that $H^{i}(X, j)$ is again pure of weight $i-2 j$ as a Galois module, i.e., $\mathbb{Z}_{\ell}$-sheaf on $(\operatorname{Spec} k)_{\text {ét }}(\mathrm{cf} .[\mathrm{De} 3,3.4 .11])$.

1.4. Although the cycle maps in (3) and (4) and, more generally, the cycle maps into Weil cohomology theories are already objects of interesting study and deep conjectures, namely, the Hodge conjecture, the Tate conjecture [Ta], and the standard conjectures [K13], this only covers a small part of the Chow groups, namely, cycles modulo homological equivalence. Recall that a cycle $\alpha \in Z^{j}(X)$ is called: 
(i) homologically equivalent to zero $\left(\alpha \sim_{\text {hom }} 0\right)$, if $\operatorname{cl}(\alpha)=0$ for a cycle map $\mathrm{cl}$ into a Weil cohomology, and

(ii) numerically equivalent to zero $\left(\alpha \sim_{\text {num }} 0\right)$, if the intersection number $(\alpha \cdot \beta)=\operatorname{deg} \alpha \cdot \beta=0$ for all $\beta \in C H^{d-j}(X)$, if $X$ is of pure dimension $d$, say.

From these definitions it is only clear that $\alpha \sim_{\text {hom }} 0$ implies $\alpha \sim_{\text {num }} 0$, and a priori $\sim_{\text {hom }}$ depends on the chosen Weil cohomology, but according to the standard conjectures $\sim_{\text {hom }}$ and $\sim_{\text {num }}$ should coincide, which would imply the independence as well. Now $C H^{j}(X) / \sim_{\text {num }}$ is a finitely generated abelian group (cf. [KI3, 5-2]), while

$$
C H^{j}(X)_{\text {hom }}=\left\{\alpha \in C H^{j}(X) \mid c l(\alpha)=0\right\}
$$

can be huge. To a certain extent this group can be studied by secondary cycle maps, namely, the Abel-Jacobi maps.

For $k=\mathbb{C}$ this is a map

$$
c l^{\prime}: C H^{j}(X)_{0} \rightarrow \frac{H^{2 j-1}(X(\mathbb{C}), \mathbb{C})}{H^{2 j-1}(X(\mathbb{C}), \mathbb{Z}(j))+F^{j}}
$$

into the Weil-Griffiths intermediate Jacobian (cf. [Lie, p. 131]). Here $F^{j}=$ $\bigoplus_{p+q=2 j-1, p \geq j} H^{p, q}(X)$ is the $j$ th step of the Hodge filtration, $C H^{j}(X)_{0}$ is the kernel of the cycle map $c l$ in example 1.3(3), and we have normalized the lattice to be $H^{2 j-1}(X, \mathbb{Z}(j))$ instead of the more classical $H^{2 j-1}(X, \mathbb{Z})$. Note that $C H^{j}(X)_{0}$ is of finite index in $C H^{j}(X)_{\text {hom }}$. By the classical AbelJacobi theorem, $c l^{\prime}$ is an isomorphism for $j=1$.

The $\ell$-adic analogue, for $\ell \neq \operatorname{char}(k)$, is a map

$$
c l^{\prime}: C H^{j}(X)_{0} \rightarrow H^{1}\left(G_{k}, H^{2 j-1}\left(\bar{X}, \mathbb{Z}_{\ell}(j)\right)\right),
$$

which can be defined by the cycle map into $H^{2 j}\left(X, \mathbb{Z}_{\ell}(j)\right)$ and the HochschildSerre spectral sequence

$$
E_{2}^{p, q}=H^{p}\left(G_{k}, H^{q}\left(\bar{X}, \mathbb{Z}_{\ell}(j)\right)\right) \Rightarrow H^{p+q}\left(X, \mathbb{Z}_{\ell}(j)\right)
$$

(cf. [Ja1, 6.15c)]). Here $C H^{j}(X)_{0}$ is the kernel of the cycle map into $H^{2 j}\left(\bar{X}, \mathbb{Z}_{\ell}(j)\right) ;$ it is of finite index in

$$
C H^{j}(X)_{\text {hom }}=\operatorname{Ker}\left(C H^{j}(X) \rightarrow H^{2 j}\left(\bar{X}, \mathbb{Q}_{\ell}(j)\right)\right) .
$$

For $j=1$ and a finitely generated field $k, c l^{\prime}$ is injective up to torsion prime to $\ell$ by Kummer theory and the Mordell-Weil theorem (cf. [Ja1, 6.15a], where "up to torsion prime to $\ell$ " should be added).

In the $\ell$-adic setting we have in addition higher than secondary cycle classes. In fact, let $F^{0} \supset F^{1} \supset \cdots$ be the filtration on $H^{2 j}\left(X, \mathbb{Q}_{\ell}(j)\right.$ ) (continuous étale cohomology over $k$ ) induced by the Hochschild-Serre spectral sequence

$$
E_{2}^{p, q}=H^{p}\left(G_{k}, H^{q}\left(\bar{X}, \mathbb{Q}_{\ell}(j)\right)\right) \Rightarrow H^{p+q}\left(X, \mathbb{Q}_{\ell}(j)\right) .
$$


Since this spectral sequence degenerates (cf. the remark in [Ja1, 6.15b]; this fact also follows from the considerations in [De5]), we have isomorphisms

$$
\operatorname{Gr}_{F}^{\nu} H^{2 j}\left(X, \mathbb{Q}_{\ell}(j)\right) \cong H^{\nu}\left(G_{k}, H^{2 j-\nu}\left(\bar{X}, \mathbb{Q}_{\ell}(j)\right) .\right.
$$

Now let $F_{\ell}$ be the descending filtration on $C H^{j}(X)$ obtained by pull-back via the cycle map

$$
c l: C H^{j}(X) \rightarrow H^{2 j}\left(X, \mathbb{Q}_{\ell}(j)\right) .
$$

Hence $F_{\ell}^{0}=C H^{j}(X), F_{\ell}^{1}=C H^{j}(X)_{\text {hom }}$, and $F_{\ell}^{2}=$ kernel of the AbelJacobi map $\mathrm{Cl}^{\prime}$, up to torsion. Then we obtain higher cycle maps

$$
c l^{(\nu)}: F_{\ell}^{\nu} C H^{j}(X) \rightarrow H^{\nu}\left(G_{k}, H^{2 j-\nu}\left(\bar{X}, \mathbb{Q}_{\ell}(j)\right)\right)
$$

with kernel $F_{\ell}^{\nu+1} C H^{j}(X)$ extending the cases $\nu=0$ and 1 discussed before.

As we remarked, $C H^{1}(X)_{\text {hom }}$ can effectively be recovered by Abel-Jacobi maps. But in any case, the structure of $C H^{j}(X)$ is well understood for $j=1$ : One has a canonical isomorphism $C H^{1}(X) \cong \operatorname{Pic}(X)$ and an exact sequence

$$
0 \rightarrow \operatorname{Pic}^{\circ}(X) \rightarrow \operatorname{Pic}(X) \rightarrow \mathrm{NS}(X / k) \rightarrow 0,
$$

where the " $k$-rational Néron-Severi group" $\mathrm{NS}(X / k)$ is a finitely generated abelian group and $\operatorname{Pic}^{\circ}(X)$ is the group of points of an abelian varietynamely, the Jacobian variety $\operatorname{Jac}(X)$-if $X$ has a rational point. In fact, $\operatorname{Jac}(X)=\left(\operatorname{Pic}_{X / k}^{\circ}\right)_{\text {red }}$ for the Picard group scheme $\operatorname{Pic}_{X / k}$ (cf. [Gr2, 3.2]), there is an exact sequence $0 \rightarrow \operatorname{Pic}(X) \stackrel{j}{\rightarrow} \operatorname{Pic}_{X / k}(k) \rightarrow \operatorname{Br}(k)$ in which $j$ is an isomorphism for $X(k) \neq \varnothing$ (cf. [Gr1, 2.1]), and we obtain the result by putting $\operatorname{Pic}^{\circ}(X)=j^{-1} \mathrm{Pic}_{X / k}^{\circ}(k)$, since $\mathrm{NS}(X / k)$ is finitely generated for algebraically closed $k$ (cf. [SGA6, XIII, 5.1]). It is known that $\operatorname{Pic}^{\circ}(X)$ has finite index in $C H^{1}(X)_{\text {num }}$.

The naive hope that similar structure results would hold also for $j \geq 2$ was destroyed by the following counterexample of Mumford. For $X$ of dimension $d$ over $k$ let $C H^{d}(X)_{0}$ be the Chow group of zero cycles of degree zero on $X$. If $k=\mathbb{C}$ (or a universal domain), then, following Mumford, call $C H^{d}(X)_{0}$ finite dimensional, if there is an $n \in \mathbb{N}$ such that the natural map

$$
\begin{aligned}
S^{n} X(k) \times S^{n} X(k) & \rightarrow C H^{d}(X)_{0}, \\
(a, b) & \rightarrow \text { class of } a-b
\end{aligned}
$$

is surjective, where $S^{n} X$ is the $n$th symmetric power of $X$.

THEOREM 1.5 [Mum1, p. 203]. Let $X$ be a smooth projective surface over C. If $H^{2}\left(X, \mathscr{O}_{X}\right) \neq 0$, then $C H^{2}(X)_{0}$ is not finite dimensional.

For the discussion of this result it is useful to investigate various possible characterizations of a "nice behaviour" of $C H^{d}(X)_{0}$. 
Proposition 1.6. Let $X$ be a smooth, projective, geometrically irreducible variety of dimension $d$ over a field $k$, and let $\Omega \supseteq k$ be an algebraically closed field. Consider the following statements.

(i) (resp. $\left.\left(\mathrm{i}^{\prime}\right)\right)$ There is an $n \in \mathbb{N}$ such that the natural map

$$
S^{n} X(\Omega) \times S^{n} X(\Omega) \rightarrow C H^{d}\left(X_{\Omega}\right)_{0}
$$

(resp. $\left.S^{n} X(\Omega) \times S^{n} X(\Omega) \rightarrow C H^{d}\left(X_{\Omega}\right)_{0} \otimes \mathbb{Q}\right)$ is surjective, where $X_{\Omega}=X \times_{k}$ $\Omega$.

(ii) (resp. (ii')) There exists a smooth projective curve $C$ over $\Omega$ and a morphism $f: C \rightarrow X_{\Omega}$ such that

$$
f_{*}: \operatorname{Pic}^{0}(C)=C H^{1}(C)_{0} \rightarrow C H^{d}\left(X_{\Omega}\right)_{0}
$$

(resp. $f_{*}: C H^{1}(C)_{0} \otimes \mathbb{Q} \rightarrow C H^{d}\left(X_{\Omega}\right)_{0} \otimes \mathbb{Q}$ ) is surjective.

(iii) (resp. (iii')) There exists a closed subscheme $Y \subseteq X$ of dimension 1 such that

$$
C H^{d}\left((X-Y)_{\Omega}\right)=0
$$

(resp. $\left.C H^{d}\left((X-Y)_{\Omega}\right)_{\mathbb{Q}}=0\right)$, where $C H^{j}(Z)$ is defined for $j \geq 0$ and any algebraic $\Omega$-scheme $Z$ by formula (1.1), applied to $Z$ over $\Omega$ in place of $X$ over $k$.

(iv) (resp. (iv')) If $B \subseteq X_{\Omega}$ is a smooth linear space section of dimension 1 , then

$$
C H^{d}\left(X_{\Omega}-B\right)=0
$$

(resp. $\left.C H^{d}\left(X_{\Omega}-B\right)_{\mathbb{Q}}=0\right)$.

(v) $\left(\right.$ resp. $\left.\left(\mathrm{v}^{\prime}\right)\right)$ The canonical map

$$
a_{X_{\Omega}}: C H^{d}\left(X_{\Omega}\right)_{0} \rightarrow \operatorname{Alb}(X)(\Omega)
$$

is an isomorphism (resp. an isomorphism after tensoring with $\mathbb{Q}$ ), where $\operatorname{Alb}(X)$ is the Albanese variety of $X$.

Then $(\mathrm{v}) \Leftrightarrow\left(\mathrm{v}^{\prime}\right) \Rightarrow$ (iv) $\Leftrightarrow\left(\mathrm{iv}^{\prime}\right) \Rightarrow$ (iii) $\Leftrightarrow\left(\right.$ iii $\left.^{\prime}\right) \Rightarrow$ (ii) $\Leftrightarrow\left(\mathrm{ii}^{\prime}\right) \Rightarrow(\mathrm{i}) \Leftrightarrow\left(\mathrm{i}^{\prime}\right)$. If $\Omega$ is uncountable, then (i) $\Rightarrow(\mathrm{v})$, so all statements are equivalent. Property (v) holds over an algebraically closed field $\Omega \supseteq k$ if and only if it holds for all algebraically closed fields $\Omega^{\prime}, \Omega \supseteq \Omega^{\prime} \supseteq k$, of finite transcendence degree over $k$. In particular, (v) holds for all algebraically closed fields $\Omega \supseteq k$, if it holds for one which is uncountable.

Proof. (v) $\Rightarrow$ (iv) Let $B \stackrel{i}{\hookrightarrow} X_{\Omega}$ be as in (iv), and consider the following commutative diagram.

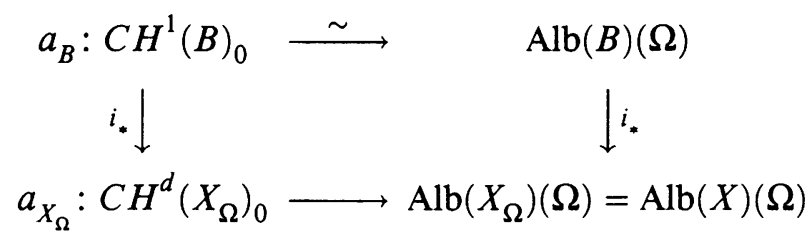


The right-hand map $i_{*}$ is surjective by the choice of $B$ (cf. [Wei, Corollary 1 to Theorem 7]). The canonical map $a_{B}$ is an isomorphism, since $\operatorname{Jac}(C)=\operatorname{Alb}(C)$ for every smooth projective curve $C$. Hence $(\mathrm{v})$ implies that the left-hand map $i_{*}$ is surjective as well, and then the same is true for $i_{*}: C H^{1}(B) \rightarrow C H^{d}\left(X_{\Omega}\right)$, since $B$ has a point of degree 1. Now (iv) follows from the exact sequence

$$
C H^{1}(B) \stackrel{i}{\rightarrow} C H^{d}\left(X_{\Omega}\right) \rightarrow C H^{d}\left(X_{\Omega}-B\right) \rightarrow 0
$$

(cf. 3.10(2)).

(iv) $\Rightarrow$ (iii) There always exists a linear section $B$ as in (iv) that is defined over a finite separable extension of $k$, and we may take $Y=$ image of $B$ in $X$ (which is not necessarily smooth). Since $B$ is a closed subvariety of $Y_{\Omega}$, the natural restriction $C H^{d}\left(X_{\Omega}-B\right) \rightarrow C H^{d}\left(X_{\Omega}-Y_{\Omega}\right)$ (cf. 3.10(1)) is surjective; hence the implication.

(iii) $\Rightarrow$ (ii) We may assume that $Y$ is of pure dimension 1 and then take $C=$ normalization of $\left(Y_{\Omega}\right)_{\text {red }}$. In fact, (iii) implies that the first map in the exact sequence

$$
C H^{1}\left(Y_{\Omega}\right) \rightarrow C H^{d}\left(X_{\Omega}\right) \rightarrow C H^{d}\left(X_{\Omega}-Y_{\Omega}\right) \rightarrow 0
$$

(cf. 3.10(2)) is surjective, but it is easy to see that the map $f_{*}$ in (ii) induced by $f: C \rightarrow\left(Y_{\Omega}\right)_{\text {red }} \rightarrow Y_{\Omega} \rightarrow X_{\Omega}$ factors as

$$
f_{*}: C H^{1}(C) \rightarrow C H^{1}\left(\left(Y_{\Omega}\right)_{\text {red }}\right)=C H^{1}\left(Y_{\Omega}\right) \rightarrow C H^{1}\left(X_{\Omega}\right),
$$

where the first map is induced by $C^{(1)} \rightarrow Y_{\Omega}^{(1)}, x \mapsto f(x)$ and, hence, is surjective.

(ii) $\Rightarrow$ (i) If $C$ is an irreducible smooth projective curve of genus $g$ over $\Omega$, then it follows from the Riemann-Roch theorem that $S^{g} C(\Omega) \times$ $S^{g} C(\Omega) \rightarrow C H^{1}(C)_{0}$ is surjective (note that $S^{n} C(\Omega)$ is identified with the set of effective divisors of degree $n$ ). Hence the claim follows from the commutative diagram

$$
\begin{array}{ccc}
S^{n} X(\Omega) \times S^{n} X(\Omega) & \rightarrow & C H^{d}\left(X_{\Omega}\right)_{0} \\
\uparrow f_{*} & & \uparrow f_{*} \\
S^{n} C(\Omega) \times S^{n} C(\Omega) & \rightarrow & C H^{1}(C)_{0}
\end{array}
$$

That (i) implies (v), for an uncountable field $\Omega$, is a theorem of Roitman ([Roi1, Theorem 4, p. 585]; in Roitman's paper it is generally assumed that $\operatorname{char}(\Omega)=0$, but the proof of this result does not involve this assumption).

By another theorem of Roitman [Roi2, 3.1], $a_{X_{\Omega}}$ always induces an isomorphism

$$
\operatorname{Tor}\left(C H^{d}\left(X_{\Omega}\right)_{0}\right) \stackrel{\sim}{\rightarrow} \operatorname{Tor}(\operatorname{Alb}(X)(\Omega))
$$

on the torsion subgroups (for all smooth projective varieties $X$ of dimension $d$ over $k$ and $\Omega \supseteq k$ algebraically closed). This implies the equivalence of 
(v) and $\left(\mathrm{v}^{\prime}\right)$, since $a_{X_{\Omega}}$ is always surjective. Another consequence is that

$$
i_{*}: \operatorname{Tor}\left(C H^{1}(B)_{0}\right) \rightarrow \operatorname{Tor}\left(C H^{d}\left(X_{\Omega}\right)_{0}\right)
$$

is surjective for any smooth linear space section of dimension $1, i: B \hookrightarrow X_{\Omega}$, by the surjectivity of $i_{*}: \operatorname{Alb}(B) \rightarrow \operatorname{Alb}\left(X_{\Omega}\right)$. Since $C H^{d}\left(X_{\Omega}\right)_{0}$ is divisible, this implies the equivalence of (iv) and (iv'), and the equivalence of (iii) and (iii'), (ii) and (ii'), or (i) and ( $\left.{ }^{\prime}\right)$ follows as well, using the ingredients of the proofs of the various implications.

Finally, if $\Omega \supseteq \Omega^{\prime}$ are algebraically closed extensions of $k$ and if $a_{X_{\Omega}} \otimes \mathbb{Q}$ is injective, then so is $a_{X_{\Omega^{\prime}}} \otimes \mathbb{Q}$, since the restriction map $C H^{d}\left(X_{\Omega^{\prime}}\right)_{\mathbb{Q}} \rightarrow$ $C H^{d}\left(X_{\Omega}\right)_{\mathbb{Q}}$ is injective (cf. 3.10(4)). Conversely, since

$$
C H^{d}\left(X_{\Omega}\right)=\underset{\lim }{\longrightarrow} C H^{d}\left(X_{\Omega^{\prime}}\right),
$$

where the limit is over all algebraically closed fields $\Omega^{\prime}, \Omega \supset \Omega^{\prime} \supset k$, of finite transcendence degree over $k$ (cf. 3.10(3)), $a_{X_{\Omega}}$ is injective if $a_{X_{\Omega^{\prime}}}$ is injective for all such $\Omega^{\prime}$. This shows the remaining claims. For the last claim note that $X$ can be defined over a field $k_{0}$ which is finitely generated over the prime field and that an uncountable algebraically closed field $\Omega \supseteq k$ contains all fields $\Omega^{\prime} \supseteq k_{0}$ of finite transcendence degree over $k_{0}$.

REMARK 1.7. Condition 1.6(iii) and its generalization to $\operatorname{dim} Y \geq 1$ appears in work of Bloch and Srinivas [BS] and is further investigated in [MS] and [SaS] (cf. also §3). Condition 1.6(ii) appears in [Bl1, lecture 1, Appendix], and the equivalence of (iii) and (v) is stated without proof in [MS, 1.2(b)]. Following Bloch (cf. [Bl1, Definition (1.1); BS, p. 1238]), we shall call $C H^{d}(X)_{0}$ representable, if $a_{X_{\Omega}}$ is an isomorphism over a universal domain $\Omega \supset k$.

If $k=\mathbb{C}$, then there is an isomorphism

$$
\operatorname{Alb}(X)(\mathbb{C}) \stackrel{\sim}{\rightarrow} \frac{H^{2 d-1}(X(\mathbb{C}), \mathbb{C})}{H^{2 d-1}(X(\mathbb{C}), \mathbb{Z}(d))+F^{d}}
$$

such that the complex Abel-Jacobi map $c l^{\prime}$ on $C H^{d}(X)_{0}$ can be identified with $a_{X}$. Hence Mumford's theorem implies the following: If $X$ is a complex surface with $H^{2}\left(X, \mathscr{O}_{X}\right) \neq 0$, then the Abel-Jacobi map

$$
c l^{\prime}: C H^{2}(X)_{0} \stackrel{a_{X}}{\rightarrow} \operatorname{Alb}(X)(\mathbb{C}) \stackrel{\sim}{\rightarrow} \frac{H^{3}(X(\mathbb{C}), \mathbb{C})}{H^{3}(X(\mathbb{C}), \mathbb{Z}(2))+F^{2}}
$$

has a huge kernel. (In fact, by Roitman's isomorphism (1.8), Ker $c l^{\prime}$ is torsion-free and divisble, and hence, a $\mathbb{Q}$-vector space. If it had a finite basis, it would lie in the image of $g_{*}: \operatorname{Pic}^{0}\left(C^{\prime}\right) \rightarrow C H^{2}(X)_{0}$ for some morphism $g: C^{\prime} \rightarrow X$ of a smooth projective curve $C^{\prime}$ into $X$, and we would obtain 1.6(ii), a contradiction). Moreover, $C H^{2}(X)_{0}$ cannot be given the structure 
of (the group of points of) an abelian variety, in a reasonable way. In fact, correctly interpreted this would mean that $a_{X}$ is an isomorphism (since $a_{X}$ is universal for regular maps (cf. [BS, p. 1238]) into abelian varieties).

Bloch proposed the following converse of Mumford's theorem.

Conjecture 1.8 [B11, Lecture 1]. If $H^{2}\left(X, \mathscr{O}_{X}\right)=0$, then $a_{X}: C H^{2}(X)_{0}$ $\rightarrow \operatorname{Alb}(X)(\mathbb{C})$ is an isomorphism.

Moreover, he extended both Mumford's theorem and this conjecture to arbitrary base fields, by using $\ell$-adic cohomology. Namely, he observed the following equivalence for the surface $X / \mathbb{C}$ :

$$
\begin{aligned}
& H^{2}\left(X, \mathscr{O}_{X}\right)=0, \\
\Leftrightarrow & H^{2}(X(\mathbb{C}), \mathbb{C})=H^{1,1} \quad \text { [by Hodge theory], } \\
\Leftrightarrow & H^{2}(X(\mathbb{C}), \mathbb{C}) \text { is algebraic, i.e., generated by cycle classes of } \\
& \text { divisors [by Lefschetz's theorem]. }
\end{aligned}
$$

The last statement makes sense for any Weil cohomology, and Bloch proves Theorem 1.9 and makes Conjecture 1.10 [Bl1, Lecture 1].

THEOREM 1.9. Let $X$ be a smooth projective surface over a field $k$, and let $\Omega \supset k$ be a universal domain. If $a_{X}: C H^{2}\left(X_{\Omega}\right)_{0} \rightarrow \operatorname{Alb}(X)(\Omega)$ is an isomorphism, then $H^{2}\left(\bar{X}, \mathbb{Q}_{\ell}(1)\right)$ is algebraic for $\ell \neq \operatorname{char}(k)$.

CONJECTURE 1.10. The converse holds; i.e., if $H^{2}\left(\bar{X}, \mathbb{Q}_{\ell}(1)\right)$ is algebraic for some $\ell \neq \operatorname{char}(k)$, then $C H^{2}(X)$ is representable.

REMARKS 1.11. (a) The consideration of a universal domain or at least of a "field containing many parameters" is essential here. In fact, the homomorphism $a_{\bar{X}}: C H^{\operatorname{dim}(X)}(\bar{X})_{0} \rightarrow \operatorname{Alb}(X)(\bar{k})$ is known to be an isomorphism for finite fields [KS, $\S 9$ ] and is conjectured to be an isomorphism for number fields $k$ by Bloch and Beilinson (cf. [Be3, 5.2]).

(b) If $k=\mathbb{C}=\Omega$, then Conjectures 1.8 and 1.10 are equivalent, by the canonical comparison isomorphism between singular and étale cohomology.

(c) We have seen that $\operatorname{Ker} a_{X}=\operatorname{Ker} c l^{\prime}$ for the complex Abel-Jacobi map $c l^{\prime}$ on $C H^{d}(X)_{0}$. If $k$ is finitely generated and $\ell \neq \operatorname{char}(k)$, then one can show as well $(\mathrm{cf} .[\mathrm{Ja} 3,9.14])$ that $\left(\operatorname{Ker} a_{X}\right) \otimes \mathbb{Z}_{\ell}=\left(\operatorname{Ker} c l^{\prime}\right) \otimes \mathbb{Z}_{\ell}$ for the $\ell$-adic Abel-Jacobi map $\mathrm{cl}^{\prime}$ on $C H^{d}(X)_{0}$.

Conjecture 1.10 is known to be true for an abelian surface $X$ over a field $k$ of characteristic $p>0$. In fact, since Tate's conjecture for $H^{2}\left(\bar{X}, \mathbb{Q}_{\ell}(1)\right)$ is known by work of Zarhin [Za] and Mori [Mo], one easily sees that $H^{2}\left(\bar{X}, \mathbb{Q}_{\ell}(1)\right)$ is algebraic if and only if $X$ is isogeneous to a product of two supersingular elliptic curves. The representability of $C H^{2}(X)_{0}$ for such surfaces was proved by Maruyama and Suwa [MS, Theorem 3.2]. 
In general, Bloch proposes the following strategy. He introduces the following three-step filtration

$$
\begin{aligned}
& C H^{2}(X) \\
& \cup \mid \\
& C H^{2}(X)_{0}=\operatorname{Ker}\left(\operatorname{deg}: C H^{2}(X) \rightarrow \mathbb{Z}\right) \\
& \cup \mid \\
& T(X)=\operatorname{Ker}\left(a_{X}: C H^{2}(X)_{0} \rightarrow \operatorname{Alb}(X)(k)\right)
\end{aligned}
$$

The action of correspondences, i.e., of $C^{2}(X \times X)$, respects this filtration, and one can show

THEOREM 1.12 (cf. [Bl1, 1.11]). If the action of correspondences on $T\left(X_{\Omega}\right)$ factors through homological equivalence, then Conjecture 1.10 is true.

Finally, Bloch discusses various aspects of the mysterious relation

$$
T(X) \leftrightarrow H^{2} .
$$

In the next section we review how Beilinson "explains" this relation on the basis of mixed motives. His approach heads toward an understanding of arbitrary Chow groups (in fact, arbitrary motivic cohomology), even in the nonrepresentable case.

\section{Beilinson's formula}

In his paper on height pairings [Be3, 5.10], Beilinson stated a conjecture on mixed motivic sheaves that leads to the following explicit conjecture in which no mixed motives are mentioned.

Conjecture 2.1 (Version 1 of Beilinson's conjecture). Let $k$ be a field. For every smooth projective variety $X$ over $k$ there exists a descending filtration $F$ on $C H^{j}(X)_{\mathbb{Q}}$, for all $j \geq 0$, such that

(a) $F^{0} C H^{j}(X)_{\mathbb{Q}}=C H^{j}(X)_{\mathbb{Q}}, F^{1} C H^{j}(X)_{\mathbb{Q}}=C H^{j}(X)_{\text {hom, } \mathbb{Q}}$, for some fixed Weil cohomology $H^{*}(X)$;

(b) $F^{r} C H^{i}(X)_{\mathbb{Q}} \cdot F^{s} C H^{j}(X)_{\mathbb{Q}} \subseteq F^{r+s} C H^{i+j}(X)_{\mathbb{Q}}$ under the intersection product;

(c) $F^{*}$ is respected by $f^{*}$ and $f_{*}$ for morphisms $f: X \rightarrow Y$;

(d) (assuming the algebraicity of the Künneth components of the diagonal) $\mathrm{Gr}_{F}^{\nu} C H^{j}(X)_{\mathbb{Q}}$ depends only on the motive modulo homological equivalence $h^{2 j-\nu}(X)$; and

(e) $F^{\nu} C H^{j}(X)_{\mathbb{Q}}=0$ for $\nu \gg 0$.

The meaning of (d) is as follows. By (b) and (c) the action of correspondences respects the filtration $F^{\circ}$, and by (a) (applied to $X \times X$ ) the induced action on $\operatorname{Gr}_{F}^{\nu} C H^{j}(X)_{\mathbb{Q}}=F^{\nu} C H^{j}(X)_{\mathbb{Q}} / F^{\nu+1} C H^{j}(X)_{\mathbb{Q}}$ factors through homological equivalence (i.e., $C H^{d}(X \times X)_{\text {hom }}$ acts as zero). For defining the 
motive $h^{i}(X)$ we have to assume that the Künneth components

$$
\pi_{i} \in H^{2 d-i}(X) \otimes H^{i}(X), \quad d=\operatorname{dim} X,
$$

of the diagonal $\Delta$ are algebraic $([\mathbf{K} \mathbf{3}$, conjecture $C(X)]$; this is a fairly weak consequence of the standard conjectures or the Tate conjecture (cf. [Ta, §3]) and holds for varieties over finite fields [KM, Theorem 2]). This means that the idempotent

$$
H^{*}(X) \rightarrow H^{i}(X) \rightarrow H^{*}(X)
$$

is given by an algebraic correspondence (again denoted by $\pi_{i}$ ) which is unique and an idempotent in $C H^{d}(X \times X)_{\mathbb{Q}} / \sim_{\text {hom }}$. The motive $h^{i}(X)$ can then be defined as the triple $\left(X, \pi_{i}, 0\right)$ (cf. [Scho]), and (d) means that

$$
\pi_{i} \mid \operatorname{Gr}_{F}^{\nu} C H^{j}(X)_{\mathbb{Q}}=\delta_{i, 2 j-\nu} \cdot \mathrm{id},
$$

where $\delta_{a, b}$ is the Kronecker symbol.

Let us write this out in more detail, for further reference. Recall [Scho] that the category $\mathscr{M}_{k}^{\sim}$ of motives over $k$ modulo some adequate equivalence relation $\sim$ can be defined as follows. Objects are triples $(X, p, m)=$ $(X, p, m)_{\sim}$, where $X$ is smooth projective over $k, p^{2}=p \in \operatorname{Corr}^{0}(X, X)$ is an idempotent, and $m \in \mathbb{Z}$, and we have

$$
\operatorname{Hom}((X, p, m),(Y, q, n))=q \operatorname{Corr}^{n-m}(X, Y) p .
$$

Here $\operatorname{Corr}^{r}(X, Y)=\bigoplus_{i} C H^{d_{i}+r}\left(X_{i} \times Y\right)_{\mathbb{Q}} / \sim$, for $X=\amalg X_{i}, X_{i}$ of pure dimension $d_{i}$, is the group of correspondences of degree $r$, and composition is the one of correspondences recalled in $\S 1$. If $h(X)=(X, \mathrm{id}, 0)_{\text {hom }}$ is the motive modulo homological equivalence associated to $X$, then

$$
h(X)=\bigoplus_{i=0}^{2 d} h^{i}(X)
$$

since the $\pi_{i}$ are pairwise orthogonal idempotents in $C H^{d}(X \times X)_{\mathbb{Q}} / \sim_{\text {hom }}=$ End $h(X)$ and

$$
\operatorname{End} h(X)=\bigoplus_{i=0}^{2 d} \operatorname{End} h^{i}(X)=\bigoplus_{i=0}^{2 d} \pi_{i} C H^{d}(X \times X)_{\mathbb{Q}} / \sim_{\text {hom }} \pi_{i},
$$

since the $\pi_{i}$ are central. Now (2.1) and (d) both mean that the action of End $h(x)$ on $\mathrm{Gr}_{F}^{\nu} C H^{j}(X)_{\mathbb{Q}}$ factors through the quotient End $h^{i}(X)$.

One would in fact expect the following stronger form of 2.1 to be true:

Strong Conjecture 2.1. This is the same as Conjecture 2.1, except that (e) is replaced by

(strong e) $\quad F^{j+1} \mathrm{CH}^{j}(X)_{\mathbb{Q}}=0$.

LEMMA 2.2. If the standard conjecture of Lefschetz type $B(X)$ is true, then Conjecture 2.1 implies its strong form. 
Proof (Compare [Ja3, 11.2]). By $B(X)$ there is a hard Lefschetz isomorphism of motives

$$
L^{d-s}: h^{s}(X) \stackrel{\sim}{\rightarrow} h^{2 d-s}(X)(d-s) \quad(s \leq d),
$$

where $M(n)$ is the $n$-fold Tate twist of a motive (in the notation of [Scho] we have $M(n)=M \otimes \mathbb{L}^{\otimes-n}$ and $\left.h^{2 d-s}(X)(d-s)=\left(X, \pi_{2 d-s}, d-s\right)\right)$. Hence $\pi_{s}: h(X) \rightarrow h^{s}(X) \rightarrow h(X)$ factors as a composition $\pi_{s}=\alpha^{\prime} \circ \alpha$, where $\alpha \in \pi_{2 d-s} C H^{2 d-s}(X \times X)_{\mathbb{Q}} / \sim_{\text {hom }}=\operatorname{Hom}\left(h(X), h^{2 d-s}(X)(d-s)\right)$ and $\alpha^{\prime} \epsilon$ $C H^{s}(X \times X)_{\mathbb{Q}} / \sim_{\text {hom }}=\operatorname{Hom}\left(h^{2 d-s}(X)(d-s), h(X)\right)$. If $\alpha$ is represented by an element $\Gamma \in C H^{2 d-s}(X \times X)_{\mathbb{Q}}$, then $\Gamma$ maps $C H^{j}(X)_{\mathbb{Q}}$ to $C H^{d-s+j}(X)_{\mathbb{Q}}$ :

$$
C H^{j}(X)_{\mathbb{Q}} \stackrel{\mathrm{pr}_{i}^{*}}{\rightarrow} C H^{j}(X \times X)_{\mathbb{Q}} \stackrel{\Gamma}{\rightarrow} C H^{2 d-s+j}(X \times X)_{\mathbb{Q}} \stackrel{\left(\mathrm{pr}_{2}\right)}{\rightarrow} C H^{d-s+j}(X)_{\mathbb{Q}},
$$

and this group vanishes for $s<j$. By (2.1), we conclude that $\mathrm{Gr}_{F}^{\nu} C H^{j}(X)_{\mathbb{Q}}$ $=0$ for $\nu>j$, hence the result. We note that Lemma 2.2 makes sense and stays true, if we restrict the consideration of Conjecture 2.1 to $X$ and $X \times X$.

A relation with mixed motives is given by the following version of Beilinson's conjecture. From now on, let $\mathscr{M}_{k}=\mathscr{M}_{k}^{\text {hom }}$ be the category of motives over $k$ modulo homological equivalence. We sometimes call these Grothendieck motives, since they are the object of his standard conjectures, but, of course, Grothendieck also defined and considered other variants.

CONJECTURE 2.3 (Version 2 of Beilinson's conjecture). This is the same as Conjecture 2.1, except that (d) is replaced by

$\left(\mathrm{d}^{\prime}\right)$ (assumptions as in Conjecture 2.1(d)) There is an abelian category $\mathscr{M} \mathscr{M}_{k}$ (of "mixed motives over $k$ ") containing the category $\mathscr{M}_{k}$ of Grothendieck motives as a full subcategory, and a functorial isomorphism

$$
\operatorname{Gr}_{F}^{\nu} C H^{j}(X)_{\mathbb{Q}} \cong \operatorname{Ext}_{\mathscr{M K}_{k}}^{\nu}\left(1, h^{2 j-\nu}(X)(j)\right),
$$

where $1=h(\operatorname{Spec} k)$ is the trivial motive.

In the following we call (2.3) "Beilinson's formula". It makes more evident and precise (granting the existence of $\mathscr{M M}_{k}$ !) how $\operatorname{Gr}_{F}^{\nu} C H^{j}(X)_{\mathbb{Q}}$ depends on the motive $h^{2 j-\nu}(X)$. Note that the category $\mathscr{M}_{k}$ is expected to be semisimple, so there are no nontrivial (Yoneda) extension groups $\operatorname{Ext}_{\mathscr{K}_{k}}^{i}(-,-)$ for $i \geq 1$. The existence of nontrivial extensions is a specific feature of the "mixed" situation.

2.4. Let us consider Beilinson's formula for $\nu=0$. Since 1 and $h^{2 j}(X)(j)$ are in $\mathscr{M}_{k}$, the assumption that $\mathscr{M}_{k}$ is a full subcategory of $\mathscr{M}_{\mathscr{M}_{k}}$ means that we have an isomorphism

$$
\operatorname{Hom}_{\mathscr{M}_{k}}\left(1, h^{2 j}(X)(j)\right) \stackrel{\sim}{\rightarrow} \operatorname{Hom}_{\mathscr{M K}_{k}}\left(1, h^{2 j}(X)(j)\right) .
$$


On the other hand, by (a) we want

But the equality

$$
\mathrm{Gr}_{F}^{0} C H^{j}(X)_{\mathbb{Q}}=C H^{j}(X)_{\mathbb{Q}} / \sim_{\text {hom }} .
$$

$$
C H^{j}(X)_{\mathbb{Q}} / \sim_{\text {hom }}=\operatorname{Hom}_{\mathscr{A}_{k}}\left(1, h^{2 j}(X)(j)\right)
$$

holds by construction of the category $\mathscr{M}_{k}$; by definition, the left-hand side is $\operatorname{Hom}_{\mathscr{M}_{k}}(1, h(X)(j))$, and this is equal to $\operatorname{Hom}_{\mathscr{M}_{k}}\left(1, h^{2 j}(X)(j)\right)$, since $\pi_{i} C H^{j}(X)_{\mathbb{Q}} / \sim_{\text {hom }} \subseteq \pi_{i} H^{2 j}(X)=0$ for $i \neq 2 j$.

For $\nu=1$ we want a map

$$
F^{1} C H^{j}(X)_{\mathbb{Q}}=C H^{j}(X)_{\text {hom }, \mathbb{Q}} \rightarrow \operatorname{Ext}_{\mathscr{M} \mathscr{K}_{k}}^{1}\left(1, h^{2 j-1}(X)(j)\right)
$$

whose kernel is $F^{2} C H^{j}(X)_{\mathbb{Q}}$. While a category $\mathscr{M M}_{k}$ as in $\left(\mathrm{d}^{\prime}\right)$ has not yet been identified (but cf. [Li] for a discussion of several proposals), we have already encountered the reflection of (2.4) in cohomology. For example, consider the $\ell$-adic cohomology, $\ell \neq \operatorname{char}(k)$. By the well-known isomorphisms

$$
H^{\nu}\left(G_{k}, V\right) \cong \operatorname{Ext}_{G_{k}}^{\nu}\left(\mathbb{Q}_{\ell}, V\right)
$$

for any (finite-dimensional) $\mathbb{Q}_{\ell}$-representation $V$ of $G_{k}$, we can in fact reinterprete the $\ell$-adic Abel-Jacobi map (after tensoring with $\mathbb{Q}$ and $\mathbb{Q}_{\ell}$ ) as a morphism

$$
c l^{\prime}: C H^{j}(X)_{\text {hom }, \mathbb{Q}} \rightarrow \operatorname{Ext}_{G_{k}}^{1}\left(\mathbb{Q}_{\ell}, H^{2 j-1}\left(\bar{X}, \mathbb{Q}_{\ell}\right)(j)\right),
$$

the $\ell$-adic version of (2.4). If $k=\mathbb{C}$, then we obtain something similar in the Hodge theory; particularly, if MHS is the category of mixed Hodge structures [De2, 2.3; St], then Carlson [Ca, Proposition 2] and Beilinson [Be2, 1.7] have constructed an isomorphism

$$
\operatorname{Ext}_{\mathrm{MHS}}^{1}(\mathbb{Z}, H)=W_{0} H_{\mathbb{C}} /\left(W_{0} H+F^{0} W_{0} H_{\mathbb{C}}\right)
$$

for a mixed Hodge structure $H$, where $W$. is the ascending weight filtration. The same formula holds for $\operatorname{Ext}_{A-\mathrm{MHS}}^{1}(A, H)$, where $A$ is $\mathbb{Q}$ or $\mathbb{R}, A$-MHS is the category of $A$-Hodge structures, and $H$ is in $A$-MHS (loc. cit.). Since $H^{2 j-1}(X(\mathbb{C}), \mathbb{Q}(j))$ is of weight $-1<0$, we can thus regard the complex Abel-Jacobi map (after tensoring with $\mathbb{Q}$ ) as a morphism

$$
c l^{\prime}: C H^{j}(X)_{\text {hom }, \mathbb{Q}} \rightarrow \operatorname{Ext}_{\mathbb{Q}-\mathrm{MHS}}^{1}\left(\mathbb{Q}, H^{2 j-1}(X(\mathbb{C}), \mathbb{Q}(j))\right) \text {. }
$$

The map (2.4) should be compatible with the Abel-Jacobi maps into cohomology; i.e., these should factor as

$$
\begin{aligned}
& H^{1}\left(G_{k}, H^{2 j-1}\left(\bar{X}, \mathbb{Q}_{\ell}(j)\right)\right)=\operatorname{Ext}_{G_{k}}^{1}\left(\mathbb{Q}_{\ell}, H^{2 j-1}\left(\bar{X}, \mathbb{Q}_{\ell}(j)\right)\right) \\
& C H^{j}(X)_{\text {hom }, \mathbb{Q}} \searrow \quad \stackrel{(2.4)}{\rightarrow} \quad \operatorname{Ext}_{\mathscr{M K}_{k}}^{1}\left(1, h^{2 j-1}(X)(j)\right) \\
& \frac{H^{2 j-1}(X(\mathbb{C}), \mathbb{C})}{H^{2 j-1}(X(\mathbb{C}), \mathbb{Q}(j))+F^{j}}=\operatorname{Ext}_{\mathbb{Q}-\mathrm{MHS}}^{1}\left(\mathbb{Q}, H^{2 j-1}(X, \mathbb{Q}(j))\right) .
\end{aligned}
$$


Here the vertical maps should be induced by exact, faithful "realization" functors

$$
\begin{aligned}
& H_{\ell}: \mathscr{M} \mathscr{M}_{k} \rightarrow \operatorname{Rep}\left(G_{k}, \mathbb{Q}_{\ell}\right):=\text { category of finite-dimensional } \\
& \mathbb{Q}_{\ell} \text {-representations of } G_{k}, \\
& H_{B}: \mathscr{M} \mathscr{M}_{k} \rightarrow \mathbb{Q} \text {-MHS, }
\end{aligned}
$$

which extend the existing functors $H_{\ell}$ and $H_{B}$ on $\mathscr{M}_{k}$ induced by $\ell$-adic and singular cohomology, respectively. Note that by definition we have

$$
\begin{array}{ll}
H_{\ell}(1)=\mathbb{Q}_{\ell}, & H_{\ell}\left(h^{i}(X)(j)\right)=\pi_{i} H^{i}\left(\bar{X}, \mathbb{Q}_{\ell}(j)\right)=H^{i}\left(\bar{X}, \mathbb{Q}_{\ell}(j)\right), \\
H_{B}(1)=\mathbb{Q}, & H_{B}\left(h^{i}(X)(j)\right)=H^{i}(X(\mathbb{C}), \mathbb{Q}(j)),
\end{array}
$$

where $\mathbb{Q}_{\ell}$ and $\mathbb{Q}$ are the trivial $\mathbb{Q}_{\ell}$-representation and trivial $\mathbb{Q}$-Hodge structure, respectively. Note also that the corresponding diagram for $\nu=0$,

$$
\begin{aligned}
& H^{2 j}\left(\bar{X}, \mathbb{Q}_{\ell}(j)\right)^{G_{k}}=\operatorname{Hom}_{G_{k}}\left(\mathbb{Q}_{\ell}, H^{2 j}\left(\bar{X}, \mathbb{Q}_{\ell}(j)\right)\right) \\
& C H^{j}(X)_{\mathbb{Q}} \rightarrow \quad C H^{j}(X)_{\mathbb{Q}} / \sim_{\text {hom }} \quad=\quad \operatorname{Hom}_{\mathscr{M}_{k}}\left(1, h^{2 j}(X)(j)\right) \\
& H^{2 j}(X(\mathbb{C}), \mathbb{Q}(j)) \cap H^{j, j}=\operatorname{Hom}_{\mathbb{Q}-\mathrm{HS}}\left(\mathbb{Q}, H^{2 j}(X(\mathbb{C}), \mathbb{Q}(j))\right),
\end{aligned}
$$

exists and commutes by definition.

2.5. The extensions given by Abel-Jacobi maps can in fact be constructed in a universal and geometric way: If $z \in Z^{j}(X)$ is a cycle that is homologically equivalent to zero, let $Z$ be the support of $z$ and put $U=X-Z$. For the $\ell$-adic cohomology $(\ell \neq$ char $k)$ we obtain a commutative diagram in the category of $\mathbb{Q}_{\ell}$-representations of $G_{k}$

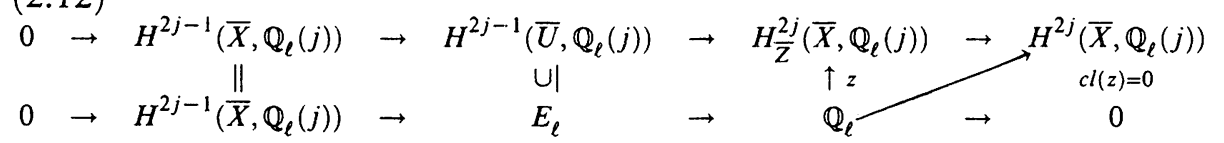

Here the exact top row is part of the localization sequence [Mi, III, 1.25], where by purity $H_{\bar{Z}}^{2 j-1}\left(\bar{X}, \mathbb{Q}_{\ell}(j)\right)=0$ and $H_{\bar{Z}}^{2 j}\left(\bar{X}, \mathbb{Q}_{\ell}(j)\right) \cong \mathbb{Q}_{\ell}^{A}$ as a $\mathbb{Q}_{\ell}{ }^{-}$ representation of $G_{k}$, where $A$ is the set of irreducible components of $\bar{Z}$. This gives the map denoted by $z$ (mapping $1 \in \mathbb{Q}_{\ell}$ to the local cycle class of $z$; cf. [Mi, VI, §9]), and the bottom exact sequence is obtained by pull-back via $z$. It is shown in $[\mathbf{J a 3}, 9.4]$ that the extension class of this sequence is the image of $z$ under the map $c l^{\prime}$ in (2.6).

An analogous result holds for the complex version (2.8), by using the corresponding diagram of mixed $\mathbb{Q}$-Hodge structures given by singular cohomology (loc. cit., 9.2 and 9.7c)). This suggests that there should exist a similar diagram in $\mathscr{M} \mathscr{M}_{k}$

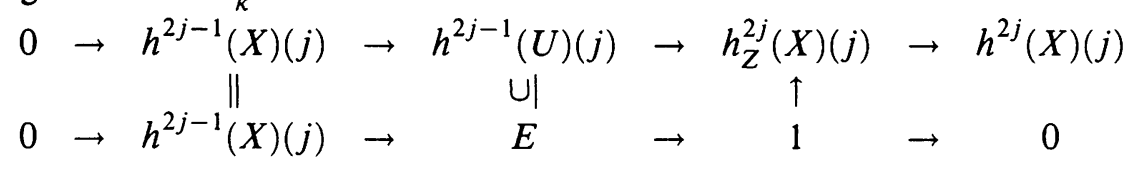


mapping to (2.12) and its Hodge analogue via $H_{\ell}$ and $H_{B}$. This consideration sheds some more light on the expected nature of $\mathscr{M M}_{k}$ and the isomorphism in Beilinson's formula. Like the target categories in (2.10), $\mathscr{M} \mathscr{M}_{k}$ should contain objects corresponding to the cohomology of arbitrary varieties over $k$. More precisely, $\mathscr{M} \mathscr{M}_{k}$ should be an abelian tensor category, and there should be a twisted Poincaré duality theory $\left((X, Z) \mapsto H_{Z}^{\cdot}(X, \cdot), X \mapsto\right.$ $H .(X, \cdot))$ in the sense of Bloch-Ogus [BO] with values in $\mathscr{M}_{\mathscr{M}}$ (cf. [Ja3, $\S 6]$ ), mapping to $\ell$-adic and singular cohomology via $H_{\ell}$ and $H_{B}$, respectively. The isomorphisms (2.3) should be compatible with the cycle maps via the realization functors (2.10). This leads to versions 3 and 4 of Beilinson's conjecture, which will be discussed in $\S 4$.

2.6. Consider the $\ell$-adic cohomology $(\ell \neq$ char $k)$. The compatibility between Beilinson's formula and the higher cycle maps for all $\nu \geq 0$ means the following. Let $F^{0} \supset F^{1} \supset \cdots$ be the filtration on $H^{2 j}\left(X, \mathbb{Q}_{\ell}(j)\right)$ introduced in 1.4. Since the action of correspondences respects the HochschildSerre spectral sequence (1.5), it respects $F^{\circ}$, and the induced action on $\operatorname{Gr}_{F}^{\nu} H^{2 j}\left(X, \mathbb{Q}_{\ell}(j)\right) \cong H^{\nu}\left(G_{k}, H^{2 j-\nu}\left(\bar{X} . \mathbb{Q}_{\ell}(j)\right)\right)$ factors through homological equivalence (for the Weil cohomology $H^{*}\left(\bar{X}, \mathbb{Q}_{\ell}(\cdot)\right)$ ). Moreover, from the above isomorphism it is clear that $\pi_{i}$ acts as $\delta_{i, 2 j-\nu} \cdot \mathrm{id}$ on this space; i.e., it depends only on $h^{2 j-\nu}(X)$. It follows that, under the cycle map

$$
c l: C H^{j}(X)_{\mathbb{Q}} \rightarrow H^{2 j}\left(X, \mathbb{Q}_{\ell}(j)\right),
$$

the conjectured filtration $F^{\cdot}$ on $C H^{j}(X)_{\mathbb{Q}}$ would map into the filtration $F^{\cdot}$ on $H^{2 j}\left(X, \mathbb{Q}_{\ell}(j)\right)$ considered here. The mentioned compatibility means the commutativity of the diagram

$$
\begin{aligned}
& \mathrm{Gr}_{F}^{\nu} C H^{j}(X)_{\mathbb{Q}} \\
& \stackrel{\mathrm{Gr}_{F}^{\nu} c l}{\rightarrow} \quad \mathrm{Gr}_{F}^{\nu} H^{2 j}\left(X, \mathbb{Q}_{\ell}(j)\right) \\
& \| \\
& \operatorname{Ext}_{\mathscr{H A}_{k}}^{\nu}\left(1, h^{2 j-\nu}(X)(j)\right) \stackrel{H_{l}}{\rightarrow} \operatorname{Ext}_{G_{k}}^{\nu}\left(\mathbb{Q}_{\ell}, H^{2 j-\nu}\left(\bar{X}, \mathbb{Q}_{\ell}(j)\right)\right)
\end{aligned}
$$

where $H_{\ell}$ associates to a Yoneda- $\nu$-extension

$$
0 \rightarrow h^{2 j-\nu}(X)(j) \rightarrow E_{\nu} \rightarrow \cdots \rightarrow E_{2} \rightarrow E_{1} \rightarrow 1 \rightarrow 0
$$

in $\mathscr{M} \mathscr{M}_{k}$ the $\nu$-extension of $\mathbb{Q}_{\ell}-G_{k}$-representations

$$
0 \rightarrow H^{2 j-\nu}\left(\bar{X}, \mathbb{Q}_{\ell}(j)\right) \rightarrow H_{\ell}\left(E_{\nu}\right) \rightarrow \cdots \rightarrow H_{\ell}\left(E_{1}\right) \rightarrow \mathbb{Q}_{\ell} \rightarrow 0 .
$$

It would be interesting to construct the $\nu$-extensions in the image of

$$
c l^{(\nu)}: F_{\ell}^{\nu} C H^{j}(X)_{\mathbb{Q}} \rightarrow \operatorname{Ext}_{G_{k}}^{\nu}\left(\mathbb{Q}_{\ell}, H^{2 j-\nu}\left(\bar{X}, \mathbb{Q}_{\ell}(j)\right)\right)
$$

(cf. 1.4) in a geometric way also for $\nu>1$. By proceeding along the lines of 2.5 one can relate these $\nu$-extensions to certain canonical extensions related to the complex computing $H^{*}\left(\bar{U}, \mathbb{Q}_{\ell}\right)$ (notation as in 2.5 ), but we do not have a geometric interpretation for these. 
The analogy with $\ell$-adic cohomology certainly provides a major motivation for the conjectures on mixed motives (cf. discussion in $\S 4$ ). The following observation provides a direct link.

LEMMA 2.7. If the cycle map

$$
\text { cl: } C H^{j}(X)_{\mathbb{Q}} \rightarrow H^{2 j}\left(X, \mathbb{Q}_{\ell}(j)\right)
$$

is injective for all $X$ in $\mathscr{V}_{k}$ and all $j \geq 0$, then Beilinson's conjecture (version 1) is true and Beilinson's filtration $F^{\cdot}$ agrees with the filtration $F_{\ell}^{*}$ of 1.4 .

Proof. Since the cycle map is compatible with the action of correspondences, the considerations in 2.6 imply that $F_{\ell}^{*}$ satisfies properties (a)-(d). The assumed injectivity implies that $\operatorname{Gr}_{F_{\ell}}^{\nu} C H^{j}(X)_{\mathbb{Q}}=0$ for $\nu \gg 0$, and the equality $F^{\cdot}=F_{\ell}^{\cdot}$ follows from the uniqueness result 5.7.

We remark that as in 2.2 it makes sense to restrict to certain subcategories of $\mathscr{V}_{k}$. Also, similar statements would hold for other suitable cycle maps into absolute cohomology theories (cf. [Ja3, 11.5]).

Of course, $\mathrm{cl}$ will not in general be injective, for example, not over an algebraically closed field. But it seems natural to ask

Question 2.8. Is $c l: C H^{j}(X)_{\mathbb{Q}} \rightarrow H^{2 j}\left(X, \mathbb{Q}_{\ell}(j)\right)$ injective for a finitely generated field $k$ ?

2.9. For the discussion of the Hodge realization, we again consider the case of zero cycles on a complex surface $X$. For the conjectural filtration we have

$$
F^{1} C H^{2}(X)_{\mathbb{Q}}=C H^{2}(X)_{0} \otimes \mathbb{Q}
$$

by definition, and since the standard conjecture $B(X)$ holds for surfaces and singular cohomology (cf. [K13, 4-3], Lemma 2.2 implies $F^{3} C H^{2}(X)_{\mathbb{Q}}=0$ (this is the argument of [B11, (1.9)]. The following lemma now implies that $F$ coincides with Bloch's filtration (1.9) after tensoring with $\mathbb{Q}$.

LEMMA 2.10. Let $X$ be a smooth, projective, irreducible variety of dimension $d$ over a field $k$. If $\operatorname{dim} H^{1}(X)=2 \operatorname{dim} \operatorname{Jac}(X)$ for the considered Weil cohomology theory (e.g., if we consider $\ell$-adic or singular cohomology, or if $B(X)$ holds), then for Beilinson's filtration $F^{*}$ we must have

$$
F^{2} C H^{d}(X)_{\mathbb{Q}}=T(X)_{\mathbb{Q}},
$$

where $T(X)$ is the kernel of $a_{X}: C H^{d}(X)_{0} \rightarrow \operatorname{Alb}(X)(k)$.

Proof. Murre constructed a certain idempotent $\tilde{\pi}_{2 d-1} \in C H^{d}(X \times X)_{\mathbb{Q}}$ (cf. [Mur1, Theorem 2]) that lifts the Künneth component $\pi_{2 d-1}$ of the diagonal under the stated assumptions on the Weil cohomology theory (loc. cit. for $\ell$-adic cohomology, hence for the singular cohomology by the comparison isomorphism; in the other cases the claim easily follows from the arguments in [Kl1, Appendix 2]). By property (2.1) of Beilinson's filtration 
we then must have $F^{2} C H^{d}(X)_{\mathbb{Q}}=$ kernel of $\tilde{\pi}_{2 d-1}$ on $C H^{d}(X)_{0} \otimes \mathbb{Q}$ (this is a special case of 5.5). But according to [Mur1, Theorem 2ii)], this kernel is $T(X)_{\mathbb{Q}}$.

Returning to our complex surface $X$, we now have

$$
\begin{gathered}
C H^{2}(X) / C H^{2}(X)_{0} \stackrel{\stackrel{c l}{\sim}}{\sim} \operatorname{Hom}_{\mathrm{HS}}\left(\mathbb{Z}, H^{4}(X(\mathbb{C}), \mathbb{Z}(2))\right), \\
C H^{2}(X)_{0} / T(X) \stackrel{c l^{\prime}}{\sim} \operatorname{Ext}_{\mathrm{MHS}}^{1}\left(\mathbb{Z}, H^{3}(X(\mathbb{C}), \mathbb{Z}(2))\right),
\end{gathered}
$$

which fits well with Beilinson's formula (2.3) for $\nu=0,1$. For $\nu=2,(2.3)$ gives

$$
T(X) \stackrel{?}{=} \mathrm{Ext}_{\mu_{M_{\mathrm{c}}}^{2}}\left(1, h^{2}(X)(2)\right),
$$

which in a way makes more precise the relation between $T(X)$ and $H^{2}$ envisioned by Bloch. Unfortunately, there is no analogue in the Hodge realization, since

$$
\operatorname{Ext}_{A-\mathrm{MHS}}^{\nu}(A,-)=0 \text { for } \nu \geq 2, A=\mathbb{Z}, \mathbb{Q} \text {, or } \mathbb{R},
$$

as easily follows from the right-exactness of $\operatorname{Ext}_{A-\mathrm{MHS}}^{1}(A,-)$. This together with Mumford's counterexample shows that the category of mixed Hodge structures is too coarse to detect all cycles or all mixed motives.

QUESTION 2.11. Is there another abelian category $(A \text {-MHS })_{\text {fine }}$ of "refined mixed Hodge structures" and a forgetful functor $F$ to $A$-MHS such that the singular cohomology $H_{B}$ factors through $F$ and there are nontrivial 2-exensions in $(A \text {-MHS })_{\text {fine }}$ ?

$(A \text {-MHS })_{\text {fine }}$ should be a rigid abelian tensor category with a weight filtration (cf. [Ja3, 6.3]), and $F$ should embed the subcategory of pure objects fully faithfully into the category $A$-HS of (pure) $A$-Hodge structures, if one believes in the classical Hodge conjecture. A possible approach could be to consider the subcategory of $A$-MHS formed by the mixed Hodge structures of "geometric origin" (cf. the considerations in [SaM2]), but this definition seems hard to handle for computational purposes.

\section{3. "Applications" and theorems}

In this section we want to show how nicely Beilinson's formula could be applied to determine Chow groups. In fact, these "applications" can also be deduced from the less fancy version 1 of Beilinson's conjecture, but the "proofs" based on Beilinson's formula are more instructive and shorter. The first two examples are only written down to illustrate this; these consequences have already been deduced from version 1 . In the following, let $X$ be smooth, projective, irreducible of dimension $d$ over $k$.

LEMMA 3.1 (cf. 2.2). If the standard conjecture of Lefschetz type $B(X)$ is true, then necessarily $F^{j+1} C H^{j}(X)_{\mathbb{Q}}=0$. 
Proof. By $B(X)$, we have a hard Lefschetz isomorphism

$$
h^{2 j-\nu}(X)(j) \cong h^{2 d-2 j+\nu}(X)(d-2 j+\nu+j) .
$$

We compute

$$
\begin{aligned}
& \operatorname{Gr}_{F}^{\nu} C H^{j}(X)_{\mathbb{Q}}=\operatorname{Ext}_{\mathscr{M M}_{k}}^{\nu}\left(1, h^{2 j-\nu}(X)(j)\right) \\
& =\operatorname{Ext}_{\text {MM }}^{\nu}\left(1, h^{2 d-2 j+2 \nu-\nu}(X)(d-j+\nu)\right) \\
& =0 \text { for } \nu>j \text {, }
\end{aligned}
$$

as a subquotient of $C H^{d-j+\nu}(X)_{\mathbb{Q}}$.

LEMMA 3.2 (cf. 1.12). Beilinson's formula implies Bloch's conjecture.

Proof. We may assume that $k$ is algebraically closed and show that the group

$$
T(X)=\operatorname{Ext}_{\text {M } \mu_{k}}^{2}\left(1, h^{2}(X)(2)\right)
$$

vanishes, if $H^{2}(X)$ is generated by algebraic cycles. Indeed, this implies that $h^{2}(X)(1)$ is a sum of trivial objects 1 , but

$$
\operatorname{Ext}_{\text {M }_{k}}^{2}(1,1(1))=0 \text {, }
$$

as a subquotient of $C H^{1}(\operatorname{Spec} k)_{\mathbb{Q}}=0$.

The next two "applications" we state as separate conjectures-which are much in the spirit of Bloch's conjecture-giving "proofs" based on Beilinson's formula. Then we shall prove actual converse theorems, in the spirit of Mumford's theorem and Bloch's generalization.

Recall Grothendieck's filtration by coniveau on the $\ell$-adic cohomology, $\ell \neq \operatorname{char} k[\mathbf{G r} 3,10.1]$ :

$$
\begin{aligned}
N^{\nu} H^{i}\left(\bar{X}, \mathbb{Q}_{\ell}\right) & =\bigcup_{Z \subseteq X} \operatorname{Im}\left(H_{\bar{Z}}^{i}\left(\bar{X}, \mathbb{Q}_{\ell}\right) \rightarrow H^{i}\left(\bar{X}, \mathbb{Q}_{\ell}\right)\right) \\
& =\bigcup_{Z \subset X} \operatorname{Ker}\left(H^{i}\left(\bar{X}, \mathbb{Q}_{\ell}\right) \rightarrow H^{i}\left(\overline{X-Z}, \mathbb{Q}_{\ell}\right)\right),
\end{aligned}
$$

where $Z$ runs over all closed subschemes of $X$ that are of codimension $\geq \nu$. The equivalence of the definitions comes from the long exact localization sequence. A variant is

$$
\tilde{N}^{\nu} H^{i}\left(\bar{X}, \mathbb{Q}_{\ell}\right)=\bigcup_{f: Y \rightarrow X} \operatorname{Im}\left(f_{*}: H^{i-2 \nu}\left(\bar{Y}, \mathbb{Q}_{\ell}(-\nu)\right) \rightarrow H^{i}\left(\bar{X}, \mathbb{Q}_{\ell}\right)\right),
$$

where $f: Y \rightarrow X$ runs over all morphisms from smooth projective varieties $Y$ of pure dimension $d-\nu$ into $X$. Obviously $\widetilde{N}^{\nu} \subseteq N^{\nu}$, and equality holds if we have resolution of singularities, e.g., if $\operatorname{char} k=0$. In fact, if $\pi: Y \rightarrow Z$ is proper and surjective, with $Y$ smooth and projective, of pure dimension $d-\nu$, then the Gysin morphism for $f: Y \stackrel{\pi}{\rightarrow} Z \stackrel{i}{\rightarrow} X$ factors as

$$
\begin{array}{ccccc}
f_{*}: H^{i-2 \nu}\left(\bar{Y}, \mathbb{Q}_{\ell}(-\nu)\right) & \rightarrow & H_{\bar{Z}}^{i}\left(\bar{X}, \mathbb{Q}_{\ell}\right) & \rightarrow & H^{i}\left(\bar{X}, \mathbb{Q}_{\ell}\right) \\
\downarrow & & & \downarrow & \\
H_{2 d-i}\left(\bar{Y}, \mathbb{Q}_{\ell}(d)\right) & \stackrel{\pi_{*}}{\rightarrow} & H_{2 d-i}\left(\bar{Z}, \mathbb{Q}_{\ell}(d)\right) & \stackrel{i^{*}}{\rightarrow} & H_{2 d-i}\left(\bar{X}, \mathbb{Q}_{\ell}(d)\right)
\end{array}
$$


so that $\operatorname{Im} f_{*} \subseteq \operatorname{Im}\left(H \frac{i}{Z}\left(\bar{X}, \mathbb{Q}_{\ell}\right)\right)$, and we have $\operatorname{Im} f_{*}=\operatorname{Im} i_{*}$ by weight arguments: Since $H^{i}\left(\bar{X}, \mathbb{Q}_{\ell}\right)$ is pure of weight $i$, the image of $i_{*}$ equals the image of $W_{i} H_{2 d-i}\left(\bar{Z}, \mathbb{Q}_{\ell}(d)\right)$, but $H_{2 d-i}\left(\bar{Y}, \mathbb{Q}_{\ell}(d)\right)$ surjects onto this space via $\pi_{*}$ (cf. [Ja3, 7.7], where $H_{0}(X, b)$ should read $H_{a}(X, b)$ ). For ètale homology and the discussion of weights we refer to [Ja3, 6.7, 6.8.2, and 6.11.1] (for our application, the technical 6.11.1 loc. cit. for char $k=0$ can be avoided by using comparison isomorphisms with singular cohomology and Deligne's result quoted in loc. cit. 7.7).

For $k=\mathbb{C}$, we define filtrations $N^{*}$ and $\tilde{N}^{\cdot}$ on $H^{i}(X(\mathbb{C}), \mathbb{Q})$ by the same formulae as above, and by resolution of singularities we have $N^{\circ}=\tilde{N}^{\circ}$. In the following we put

$$
H^{i}(X, j)= \begin{cases}H^{i}\left(\bar{X}, \mathbb{Q}_{\ell}(j)\right) & \text { if char } k \neq \ell, \text { or } \\ H^{i}(X(\mathbb{C}), \mathbb{Q}(j)) & \text { if } k=\mathbb{C}\end{cases}
$$

to treat both cases in a parallel way. Similarly we use the notation $H_{Z}^{i}(X, j)$ for $H_{\bar{Z}}^{i}\left(\bar{X}, \mathbb{Q}_{\ell}(j)\right)$ or $H_{Z}^{i}(\mathbb{C})(X(\mathbb{C}), \mathbb{Q}(j))$, and $H_{a}(X, b)$ for $H_{a}\left(\bar{X}, \mathbb{Q}_{\ell}(b)\right)$ or $H_{a}(X(\mathbb{C}), \mathbb{Q}(b))$ (Borel-Moore homology). In fact, everything below could be extended to any suitable Poincaré duality theory with weights satisfying the axioms a)-m) of $[\mathbf{J a 3}, \S 6,7]$ and such that $H^{i}(X, j)$ defines a Weil cohomology on $V_{k}$ after applying a forgetful functor to vector spaces. Moreover, we sometimes omit Tate twists, by writing $H^{i}(X)$ for $H^{i}(X, j)$, etc.

Note that for a surface $X$ we have

$$
H^{2}(X) \text { is algebraic } \Leftrightarrow N^{1} H^{2}(X)=H^{2}(X) .
$$

Hence the following is a generalization of Bloch's conjecture.

CONJECTURE 3.3. If $H^{i}(X)$ is supported in codimension 1 (i.e., $H^{i}(X)=$ $\left.N^{1} H^{i}(X)\right)$, for $i=2, \ldots, d$, then $C H^{d}(X)_{0}$ is representable.

"Proof". Assuming Beilinson's formula, the standard conjectures, and resolution of singularities over $k$-The assumption implies that there is a smooth projective variety $Y$ of dimension $d-1$ and an epimorphism

$$
h^{i-2}(Y)(-1) \rightarrow h^{i}(X), \quad i=2, \ldots, d .
$$

By Poincaré duality this gives a monomorphism

$$
h^{2 d-i}(X)(d) \hookrightarrow h^{2 d-i}(Y)(d), \quad i=2, \ldots, d .
$$

Since $\mathscr{M}_{k}$ is semisimple, we obtain an induced injection

$$
\begin{array}{ccc}
\operatorname{Ext}_{\mathscr{M K _ { k }}}^{\nu}\left(1, h^{2 d-\nu}(X)(d)\right) & \hookrightarrow \operatorname{Ext}_{\mathscr{M K _ { k }}}^{\nu}\left(1, h^{2 d-\nu}(Y)(d)\right) \\
\operatorname{Gr}_{F}^{\nu} C H^{d}(X)_{\mathbb{Q}} & & \operatorname{Gr}_{F}^{\nu} C H^{d}(Y)_{\mathbb{Q}}=0
\end{array}
$$


for $\nu \geq 2$ and obtain

$$
0=F^{2} C H^{d}(X)_{\mathbb{Q}}=T(X)_{\mathbb{Q}},
$$

where $T(X)=\operatorname{Ker}\left(a_{X}: C H^{d}(X)_{0} \rightarrow \operatorname{Alb}(X)(k)\right)$ (cf. 2.10). The same reasoning shows that $T\left(X_{E}\right)=0$ over any algebraically closed extension field $E$ of $k$ (since the surjection (3.3) carries over to the base extensions $Y_{E}, X_{E}$ ), hence the result. Note that we need Beilinson's formula for $X$ and $Y$ over all such $E$ or over a universal domain $\Omega \supset k$.

Conjecture 3.4. If $H^{\circ}(X)$ is algebraic, then $C H^{\circ}\left(X_{E}\right)_{\text {num }} \otimes \mathbb{Q}=0$ over any extension field $E$ of $k$.

"ProoF". Assuming Beilinson's formula-We may assume that $k$ is algebraically closed. Then the assumption means that

$$
H^{i}(X)= \begin{cases}0 & \text { for } i \text { odd } \\ \text { generated by cycle classes } & \text { for } i \text { even. }\end{cases}
$$

This implies that $\sim_{\text {hom }} \sim_{\text {num }}$ and that the standard conjectures of Lefschetz type are true for $X$. From this we easily deduce that

$$
h^{2 j-\nu}(X)(j)= \begin{cases}0 & \text { if } \nu \text { is odd }, \\ \text { sum of copies of } 1(\mu) & \text { if } \nu=2 \mu \text { is even },\end{cases}
$$

for all $j$ and $\nu$. But

$$
\operatorname{Ext}_{\mathscr{M N _ { k }}}^{2 \mu}(1,1(\mu))=C H^{\mu}(\operatorname{Spec} k)_{\mathbb{Q}}=0 \text { for } \mu>0
$$

showing that $F^{1} C H^{j}(X)_{\mathbb{Q}}=0$ for all $j \geq 0$, and the same is true over all extension fields.

The cohomology is known to be algebraic for the standard cellular varieties, like projective spaces, Grassmannians, Schubert varieties, etc., but for these it is also known that $C^{\circ}(X)_{\text {num }} \otimes \mathbb{Q}=0$ ! We now come to the converse theorems, showing that the conditions in 3.3 and 3.4 are in fact necessary.

THEOREM 3.5. (a) If $C H^{d}(X)_{0}$ is representable, then $H^{i}(X)$ is supported in codimension 1 , for $i=2, \ldots, d$.

(b) More generally, say that $C H^{d}(X)_{0}$ has rank $\leq \mu(0 \leq \mu \leq d)$, if there is a closed subscheme $Z \subset X$ of dimension $\mu$ such that the restriction

$$
C H^{d}\left(X_{\Omega}\right)_{\mathbb{Q}} \rightarrow C H^{d}\left((X-Z)_{\Omega}\right)_{\mathbb{Q}}
$$

is zero, where $\Omega \supset k$ is a universal domain (e.g., $\Omega=k$, if $k=\mathbb{C}$ ). If $C H^{d}(X)_{0}$ has rank $\leq \mu$, then $H^{i}(X)$ is supported in codimension 1 for $i=\mu+1, \ldots, d$.

THEOREM 3.6. (a) If $C H^{*}\left(X_{\Omega}\right)_{\text {hom }} \otimes \mathbb{Q}=0$ for a universal domain $\Omega \supset k$, then $H^{*}(X)$ is algebraic. 
(b) If $C H^{\nu}\left(X_{\Omega}\right)_{\mathrm{hom}} \otimes \mathbb{Q}=0$ for $\nu=i, \ldots, d$, then $H^{\mu}(X)$ is algebraic for $\mu \leq 2(d-i+1)$ and $H^{\mu}(X)=N^{d-i+1} H^{\mu}(X)$ for $\mu \geq 2(d-i+1)$.

The two theorems will be proved later in 3.10. Similar results have been obtained independently by Lewis [Le], Schoen [Schoe], Colliot-Thélène, Raskind, and Saito [CTRS], and S. Saito [SaS]. In particular, we refer to the last paper for many generalizations and a thorough discussion of the relation between filtrations on Chow groups, the coniveau filtration, and the ranks of Chow groups. All these papers consider only fields of characteristic zero (to use resolution of singularities), and Theorem 3.6 is only obtained conditionally (assuming the Hodge conjecture or some standard conjecture). Theorem 3.6(a) improves a result of Bloch (cf. [K12, 3.12]).

We note that in Theorems 3.5 and 3.6 we may replace the coniveau filtration by the following, more concrete filtrations: Following Grothendieck $[\mathbf{G r 3}, 10.2 ; \mathbf{G r} 4]$, define the filtrations

$$
\begin{aligned}
N_{H}^{\nu} H^{i}(X(\mathbb{C}), \mathbb{Q})= & \text { the union of sub-Hodge structures } H \text { of } \\
& H^{i}(X(\mathbb{C}), \mathbb{Q}) \text { for which } H(\nu) \text { is effective }
\end{aligned}
$$

for $k=\mathbb{C}$, and

$$
\begin{aligned}
N_{\ell}^{\nu} H^{i}\left(\bar{X}, \mathbb{Q}_{\ell}\right)= & \text { the union of sub- } G_{k} \text {-representations } V \text { of } \\
& H^{i}\left(\bar{X}, \mathbb{Q}_{\ell}\right) \text { for which } V(\nu) \text { is effective }
\end{aligned}
$$

if $k$ is finitely generated and $\ell \neq k$. A Hodge structure is effective, if $p \geq 0$ and $q \geq 0$ for the occurring Hodge types $(p, q)$, and a pure $G_{k^{-}}$ representation $V$ is effective if the same is true for the " $\ell$-adic Hodge numbers" (as defined by Deligne [De3, 3.3.7]) of the Frobenius eigenvalues at all good places. Equivalently, $V$ is effective, if $V$ and $\operatorname{Hom}\left(V, \mathbb{Q}_{\ell}(-w)\right)$ are both entire, for $w=$ weight of $V$, where a $G_{k}$-representation $W$ is called entire, if the Frobenius eigenvalues at good places are algebraic integers. Then one has

$$
N^{\nu} H^{i}(X(\mathbb{C}), \mathbb{Q}) \subseteq N_{H}^{\nu} H^{i}(X(\mathbb{C}), \mathbb{Q}),
$$

and the generalized Hodge conjecture [Gr4] predicts that equality holds. Similarly

$$
N^{\nu} H^{i}\left(\bar{X}, \mathbb{Q}_{\ell}\right) \subseteq N_{\ell}^{\nu} H^{i}\left(\bar{X}, \mathbb{Q}_{\ell}\right),
$$

and equality holds by the generalized Tate conjecture (cf. [Gr3, 10.3; Ja3, 10.2b]). Note

$$
\begin{aligned}
& H^{i}(X(\mathbb{C}), \mathbb{Q})=N_{H}^{\nu} H^{i}(X(\mathbb{C}), \mathbb{Q}) \\
\Leftrightarrow & p \geq \nu, q \geq \nu \text { for the occurring Hodge types }(p, q),
\end{aligned}
$$

similarly for the $\ell$-adic cohomology. Hence

$$
H^{i}(X(\mathbb{C}), \mathbb{Q})=N^{1} H^{i}(X(\mathbb{C}), \mathbb{Q}) \Rightarrow H^{0}\left(X, \Omega^{i}\right)=0,
$$

and from Theorem 3.5 we, in particular, obtain Roitman's theorem [Roi1, Theorem 3]: 
Corollary 3.7. Let $k=\mathbb{C}$. If $H^{0}\left(X, \Omega^{i}\right) \neq 0$ for some $i=2, \ldots, d$, then $\mathrm{CH}^{d}(X)_{0}$ is not representable.

This was proved by Roitman by completely different methods. For arbitrary fields we obtain

COROllaRY 3.8. Let $\ell \neq$ char $k$. If $H^{i}\left(\bar{X}, \mathbb{Q}_{\ell}\right)(1)$ is not entire, for some $i=2, \ldots, d$, then $C H^{d}(X)_{0}$ is not representable.

We note that $\operatorname{Hom}\left(H^{i}\left(\bar{X}, \mathbb{Q}_{\ell}\right), \mathbb{Q}_{\ell}(-i)\right) \cong H^{i}\left(\bar{X}, \mathbb{Q}_{\ell}\right)$ by Poincaré duality and hard Lefschetz. Hence $H^{i}\left(\bar{X}, \mathbb{Q}_{\ell}\right)(\nu)$ is effective if and only if it is entire. We now prove Theorems 3.5 and 3.6, by refining Bloch's method for surfaces.

Proposition 3.9. Let $z$ be a cycle of codimension $d$ on $X \times X$.

(a) If $z$ is supported on $X \times Y$, for $Y \subset X$ of codimension $\mu$, then $z$ as a correspondence maps $H^{i}(X)$ to $N^{\mu} H^{i}(X)$ for all $i$.

(b) If $z$ is supported on $Y^{\prime} \times X$, for $Y^{\prime} \subset X$ of dimension $\nu$, then $z$ as a correspondence maps $H^{i}(X)$ to 0 for $i>2 \nu$ and to $N^{1} H^{i}(X)$ for $\nu<i \leq 2 \nu$.

Proof. Without loss of generality we may assume that $z=Z$ is a prime cycle, i.e., $i: Z \hookrightarrow X \times X$ closed, integral, of dimension $d$. Define the maps

$$
X \stackrel{f_{1}}{\leftarrow} Z \stackrel{f_{2}}{\rightarrow} X
$$

by $f_{j}=\operatorname{pr}_{j} i$, where $\operatorname{pr}_{1}$ and $\operatorname{pr}_{2}: X \times X \rightarrow X$ are the first and second projection, respectively. Then the correspondence $Z$ is the composition

$$
H^{i}(X) \stackrel{f_{1}^{*}}{\rightarrow} H^{i}(Z) \stackrel{\alpha_{Z}}{\rightarrow} H_{2 d-i}(Z) \stackrel{\left(f_{2}\right)_{*}}{\rightarrow} H_{2 d-i}(X) \stackrel{\alpha_{X}^{-1}}{\underset{\sim}{\longrightarrow}} H^{i}(X)
$$

where $\alpha_{Z}$ and $\alpha_{X}$ are the canonical maps, obtained by cap-product with the fundamental classes $\eta_{Z}$ and $\eta_{X}$ respectively (cf. [Ja3, 6.1i), j)]). This follows from the projection formula and the compatibility of cup- and capproduct, making the following diagram commute:

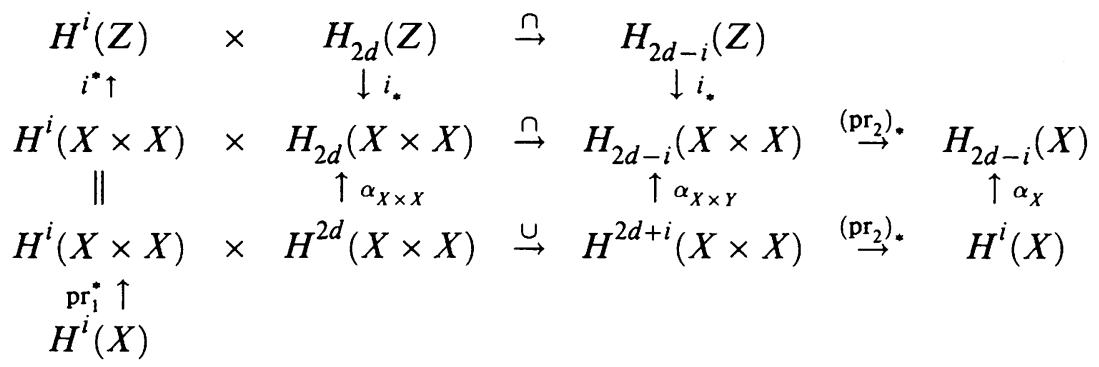

where $\left(\alpha_{X \times X}\right)^{-1} i_{*}$ maps $\eta_{Z}$ to the class of $Z$ in $H^{2 d}(X \times X)$.

Then (a) follows from the fact that $f_{2}$ factors as $Z \rightarrow Y \rightarrow X$, so that the correspondence $Z$ factors through $H_{2 d-i}(Y) \stackrel{\sim}{\rightarrow} H_{Y}^{i}(X)$. Now consider 
(b) (cf. [Ja3, proof of Theorem 10.1]): One shows the existence of a diagram

$$
\begin{array}{r}
U^{\prime} \leftarrow \quad \leftarrow \stackrel{g_{1}}{\rightarrow} U \\
\cap \mid \\
X \leftarrow Y^{\prime} \leftarrow Z \stackrel{f_{1}}{\rightarrow} X
\end{array}
$$

with vertical open immersions, in which $U^{\prime}$ is affine, the composition $Z \rightarrow$ $Y^{\prime} \rightarrow X$ is $f_{1}, V=f_{1}^{-1}(U)$ so that $g_{1}$ is proper, and $U$ is nonempty. The commutative diagram

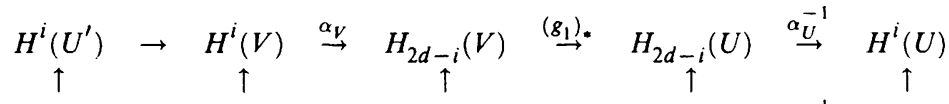

$$
\begin{aligned}
& H^{i}(X) \rightarrow H^{i}\left(Y^{\prime}\right) \rightarrow H^{i}(Z) \stackrel{\alpha_{Z}}{\rightarrow} H_{2 d-i}(Z) \stackrel{\left(f_{1}\right) .}{\rightarrow} \quad H_{2 d-i}(X) \stackrel{\alpha_{\underline{X}}^{-1}}{\rightarrow} \quad H^{i}(X)
\end{aligned}
$$

shows that the image of the correspondence $Z$ is zero in $H^{i}(X)$ if $i>$ $2 \operatorname{dim} Y^{\prime}$ (since then $H^{i}\left(Y^{\prime}\right)=0$ ) and maps to zero in $H^{i}(U)$ if $i>$ $\operatorname{dim} Y^{\prime}=\operatorname{dim} U^{\prime}$ (since then $H^{i}\left(U^{\prime}\right)=0$ by weak Lefschetz).

3.10. For the proofs of Theorems 3.5 and 3.6 we use the following four well-known facts about Chow groups.

(1) Chow groups $C H^{j}(X)$ can be defined for arbitrary Noetherian schemes $X$, as the cokernel of Quillen's divisor map

$$
\bigoplus_{y \in X^{(j-1)}} \kappa(y)^{\times} \stackrel{\text { div }}{\rightarrow} \bigoplus_{x \in X^{(j)}} \mathbb{Z}
$$

$\operatorname{div}=d_{1}^{j-1,-j}$ in Quillen's spectral sequence for algebraic $K$-theory [Qui, $\S 7$, Theorem 5.4], compare formula (1.1). They are contravariant for flat morphisms, since (3.4) is (loc. cit.). Explicitly, $\operatorname{div}(f)=\sum \operatorname{ord}_{x}(f)$ for $f \in \kappa(y)^{\times}$, where the sum is over all $x \in X^{(j)} \cap Y, Y=\overline{\{y\}}$, and $\operatorname{ord}_{x}$ is the order function of $\mathscr{O}_{Y, x}: \operatorname{ord}_{x}(f)=\operatorname{length}\left(\mathscr{O}_{Y, x} /(f)\right)$ for $f \in \mathscr{O}_{Y, x}$. Moreover, for a flat morphism $f: X^{\prime} \rightarrow X$ and $x \in X^{(j)}$ one has $f^{*}(x)=$ $\sum e\left(x^{\prime} \mid x\right) x^{\prime}$, where the sum is over all $x^{\prime} \in\left(X^{\prime}\right)^{(j)}$ with $f\left(x^{\prime}\right)=x$, and where $e\left(x^{\prime} \mid x\right)=$ length $\left(\mathscr{O}_{X^{\prime}, x^{\prime}} \otimes \mathcal{K}(x)\right)$ is the ramification index of $x^{\prime} \mid x$. (For nonequidimensional schemes, these definitions differ from those in [Fu], since we are grading by codimension instead of dimension as in [Fu]. In particular, there is no contradiction to loc. cit., Example 1.7.1.)

(2) If $X$ is algebraic over a field and $Y \subseteq X$ is a closed subscheme of pure codimension $c$, then there is an exact sequence

$$
C H^{j-c}(Y) \rightarrow C H^{j}(X) \rightarrow C H^{j}(X-Y) \rightarrow 0,
$$

induced by the obvious exact sequence

$$
0 \rightarrow \bigoplus_{x \in Y^{(j-c)}} \mathbb{Z} \rightarrow \bigoplus_{x \in X^{(j)}} \mathbb{Z} \rightarrow \bigoplus_{x \in U^{(j)}} \mathbb{Z} \rightarrow 0 .
$$


(3) If $X=\lim _{i} X_{i}$ is the projective limit of Noetherian schemes, with affine flat transition morphisms, then

$$
C H^{j}(X)=\underset{i}{\lim } C H^{j}\left(X_{i}\right),
$$

since (3.4) has this limit property [Qui, §7, Theorem 5.4].

(4) If $X$ is smooth over a field $k$, and if $L$ is any field extension of $k$, then

$$
C H^{j}(X) \rightarrow C H^{j}\left(X_{L]}\right)
$$

is injective up to torsion (cf. [Bl1, lecture 1, appendix]).

Proof of Theorem 3.5. (a) If $C H^{d}(X)_{0}$ is representable, then by Proposition 1.6 and 3.10(4) there exists a finitely generated field $k_{0} \subset k$ such that $X$ has a model $X_{0}$ over $k_{0}$ and a curve $C \subset X_{0}$ such that

$$
C H^{d}\left(\left(X_{0}-C\right)_{L}\right)_{\mathbb{Q}}=0
$$

for every field extension $L$ that is finitely generated over $k_{0}$. Looking at the restriction

$$
C H^{d}\left(X_{0} \times X_{0}\right)_{\mathbb{Q}} \rightarrow C H^{d}\left(X_{0} \times_{k_{0}} k_{0}\left(X_{0}\right)\right)_{\mathbb{Q}},
$$

where $k_{0}\left(X_{0}\right)$ is the function field of $X_{0}$ over $k_{0}$, we see from 3.10(2), (3) that we have a decomposition $\Delta=\Gamma_{1}+\Gamma^{1}$ of the diagonal in $C H^{d}\left(X_{0} \times X_{0}\right)_{\mathbb{Q}}$ such that $\Gamma_{1}$ is supported on $C \times X_{0}$ and $\Gamma^{1}$ is supported on $X_{0} \times D$ for some divisor $D$. Since $\Delta$ acts as the identity on $H^{i}(X)$, Theorem 3.5(a) immediately follows from Proposition 3.9.

(b) If $\operatorname{rk}\left(C H^{d}(X)_{0}\right)=\mu$, then the above holds with $C$ a subscheme of dimension $\mu$ instead of a curve, and the result follows from the proposition as well.

Proof OF THeORem 3.6. Obviously (a) follows from (b). We may assume that $k$ is the algebraic closure of a finitely generated field. If $C H^{d}(X)_{\text {hom }} \otimes$ $\mathbb{Q}=0$, then taking any closed point $x \in X$ we have

$$
C H^{d}\left((X-\{x\})_{k(X)}\right)_{\mathbb{Q}}=0 .
$$

As above we conclude that $\Delta=\Gamma_{0}+\Gamma^{1}$, where $\Gamma_{0}$ is supported on $\{x\} \times X$ and $\Gamma^{1}$ is supported on $X \times D$ for a divisor $D$. By the proposition, $\Gamma_{0}$ is zero on $H^{i}(X)$ for $i>0$ and $\Gamma^{1}$ maps $H^{i}(X)$ to $N^{1} H^{i}(X)$ for all $i$. Since $\Delta$ is the identity, we get

$$
\begin{aligned}
& H^{1}(X)=0 \\
& H^{i}(X)=N^{1} H^{i}(X) \text { for all } i \geq 2 .
\end{aligned}
$$

Now look at $\Gamma^{1} \in C H_{d}(X \times D)_{\mathbb{Q}}=C H^{d-1}(X \times D)_{\mathbb{Q}}$. If $D$ is irreducible, let $k(D)$ be the function field of $D$ and let $p: \operatorname{Spec} k(d) \rightarrow$ Spec $k$ be the structural map. By choosing a chain Spec $k=Z_{0} \subset Z_{1} \subset \cdots \subset Z_{d-1}=D$ of 
integral closed subvarieties $Z_{i}$ such that $Z_{i-1}$ has codimension 1 in $Z_{i}$, we get a specialization map sp

$$
C H^{d-1}(X) \underset{p^{*}}{\stackrel{s p}{\leftrightarrows}} C H^{d-1}\left(X \times_{k} k(D)\right)
$$

with $\operatorname{sp} p^{*}=$ id (cf. [DV, exp III]). This specialization map is compatible with the corresponding one in the $\ell$-adic cohomology via the cycle map (cf. [Ja3, pp. 201-202]), as indicated in the following commutative diagram.

$$
\begin{array}{ccc}
C H^{d-1}(X)_{\mathbb{Q}} & \underset{p^{*}}{\stackrel{\mathrm{sp}}{\leftrightarrows}} & C H^{d-1}\left(X \times_{k} k(D)\right)_{\mathbb{Q}} \\
c l \downarrow & \stackrel{\mathrm{sp}}{\leftrightarrows} & H^{2 d-2}\left(X \times_{k} \frac{\downarrow c l}{k(D)}, \mathbb{Q}_{\ell}(d-1)\right) \\
H^{2 d-2}\left(\bar{X}, \mathbb{Q}_{\ell}(d-1)\right) & \underset{p^{*}}{\stackrel{\mathrm{s}}{\leftrightarrows}} &
\end{array}
$$

Since $p^{*}$ and sp are isomorphisms for the $\ell$-adic cohomology [Mi, VI, 4.2 and 4.3 ], we have

$$
c l(\alpha)-\operatorname{clp} p^{*} \operatorname{sp}(\alpha)=\operatorname{cl}(\alpha)-p^{*} \operatorname{sp} c l(\alpha)=0
$$

for every $\alpha \in C H^{d-1}(X \times k(D))_{\mathbb{Q}}$. If now $c l$ is injective on this group, we deduce that $p^{*} \mathrm{sp}=$ id on $C H^{d-1}(X \times k(D))_{\mathbb{Q}}$. Hence there exists a cycle $\Gamma_{1}^{\prime}$ in $C H^{d-1}(X)_{\mathbb{Q}}$ such that $\Gamma^{1}-\Gamma_{1}^{\prime} \times D$ maps to zero in $C H^{d-1}(X \times k(D))_{\mathbb{Q}}$, i.e.,

$$
\Gamma^{1}=\Gamma_{1}+\Gamma^{2} \text { in } C H^{d}(X \times X)_{\mathbb{Q}},
$$

where $\Gamma_{1}=\Gamma_{1}^{\prime} \times D$ is supported on $Y_{1} \times X$ with $\operatorname{dim} Y_{1}=1$ and $\Gamma^{2}$ is supported on $X \times Y^{2}$ with $\operatorname{codim} Y^{2}=2$. This is also true if $D$ is not irreducible: if $D_{1}, \ldots, D_{r}$ are the irreducible components of $D$, then we can write

$$
\Gamma^{1}=\sum_{i=1}^{r} \Gamma_{i}^{1}+\widetilde{\Gamma}^{2}
$$

with $\Gamma_{i}^{1}$ supported on $X \times D_{i}$ and $\widetilde{\Gamma}^{2}$ supported on $X \times \widetilde{Y}^{2}$ with $\operatorname{codim} \widetilde{Y}^{2}=$ 2 , and apply the above to the $\Gamma_{i}^{1}$.

If $k=\mathbb{C}$, we can apply a similar argument to the singular cohomology, by specializing from generic to special points, or one can still work with $\ell$ adic cohomology, using the fact that by the comparison isomorphism with singular cohomology, homological equivalence is the same for them. Hence the above is established in both cases, and we proceed with the proof.

By the proposition, $\Gamma_{1}$ maps $H^{i}(X)$ to zero for $i>2$ and $\Gamma^{2}$ maps $H^{i}(X)$ to $N^{2} H^{i}(X)$. Since $\Gamma^{1}$ is the identity on $H^{i}(X)$ for $i>0$, we see that

$$
\begin{aligned}
& H^{3}(X)=0 \\
& H^{\mu}(X)=N^{2} H^{\mu}(X) \quad \text { for } \mu \geq 4 .
\end{aligned}
$$


Inductively, we now show: If $C H^{\nu}\left(X_{\Omega}\right)_{\text {hom, } \mathbb{Q}}=0$ for $\nu=d-i, \ldots, d$, then there are cycles of codimension $d$ on $X \times X$

$$
\begin{aligned}
\Gamma_{j} \text { supported on } Y_{j} \times X, \operatorname{dim} Y_{j} & =j, \\
\Gamma^{j} \text { supported on } X \times Y^{j}, \operatorname{codim} Y^{j} & =j,
\end{aligned}
$$

for $j=0, \ldots, i\left(\Gamma^{j}\right.$ also for $\left.j=i+1\right)$ such that $\Gamma^{0}=\Delta, \Gamma^{d+1}=0$,

$$
\Gamma^{j}=\Gamma_{j}+\Gamma^{j+1}
$$

and $\Gamma^{j}$ acts as the identity on $H^{i}(X)$ for $i>2 j-2$. By looking at $\Gamma^{j+1}$ on $H^{2 j+1}(X)$ and $H^{2 j+2}(X)$, we obtain that

$$
\begin{aligned}
& H^{-2 j+1}(X)=N^{j+1} H^{2 j+1}(X)=0, \\
& H^{2 j+2}(X)=N^{j+1} H^{2 j+2}(X)
\end{aligned}
$$

for $j=0, \ldots, i$, and by looking at $\Gamma^{i+1}$ we see that

$$
H^{\mu}(X)=N^{i+1} H^{\mu}(X), \quad \mu \geq 2 i+2 ;
$$

hence the result.

REMARKs 3.11. (a) The motivation for Theorem 3.6 came from the following potential application, in connection with results of Faber [Fa]. It should be possible to extend Theorem 3.6 to $Q$-varieties (i.e., varieties that are locally the quotient of a smooth variety by a finite group) and, in particular, to the compactifications $\overline{\mathscr{M}}_{g}$ of the moduli spaces $\mathscr{M}_{g}$ of curves of genus $g$. Then one could try to use some knowledge on $C H^{\cdot}\left(\mathscr{\mathscr { M }}_{g}\right)_{\mathbb{Q}}$ to prove that $H^{i}\left(\mathscr{M}_{g}\right)$ is generated by algebraic classes for $i \ll g$ as conjectured by Mumford [Mum3, p. 272].

(b) A variant of Theorem 3.6 was recently proved by Esnault and Levine [EL], with similar methods. They give necessary conditions for the injectivity of the cycle map into Deligne cohomology, for a smooth projective variety over $\mathbb{C}$. Also, we refer the reader to a recent paper by Paranjape [Pa], in which methods similar to those of this section have been used to check other predictions of the conjectures of Bloch and Beilinson.

We conclude this section with

Conjecture 3.12. The functor $X \mapsto C H^{j}(X)$ is of order $2 j$ on $\mathscr{V}_{k}$.

"Proof". For $C H^{j}(X)_{\mathbb{Q}}$, assuming Beilinson's formula-Recall (cf. [Mum2, p. 55]) that a contravariant functor $F$ on varieties with values in an abelian category is called of order $n$, if for any irreducible varieties $X_{0}, \ldots, X_{n}$ the morphism

$$
\sum_{i=0}^{n} p_{i}^{*}: \bigoplus_{i=0}^{n} F\left(X_{0} \times \cdots \times \widehat{X}_{i} \times \cdots \times X_{n}\right) \rightarrow F\left(X_{0} \times \cdots \times X_{n}\right)
$$


is surjective, where

$$
p_{i}: X_{0} \times \cdots \times X_{n} \rightarrow X_{0} \times \cdots \times \widehat{X}_{i} \times \cdots \times X_{n}
$$

is the projection ( $\widehat{X}_{i}$ indicating the omission of the factor $X_{i}$ in the product). If the Künneth components of the diagonal are algebraic, then the Künneth formula for the chosen Weil cohomology implies that, with the notation just introduced, $h^{n}\left(X_{0} \times \cdots \times X_{n}\right)$ is direct factor of $\bigoplus_{i=0}^{n} h^{n}\left(X_{0} \times \cdots \times \widehat{X}_{i} \times \cdots \times X_{n}\right)$ via $\sum p_{i}^{*}$. Consequently, Beilinson's formula implies that

$$
\operatorname{Gr}_{F}^{\nu} C H^{j}(X)_{\mathbb{Q}} \cong \operatorname{Ext}_{\mathscr{M} \mathscr{M}_{k}}^{\nu}\left(1, h^{2 j-\nu}(X)(j)\right)
$$

is of order $2 j-\nu$; hence the result.

We note that trivially $C H^{0}(X)$ is of order 0 , while $C H^{1}(X)$ is of order 2 by the theorem of the cube (loc. cit.). Therefore Conjecture 3.12 is sometimes called the (conjectural) theorem of the hypercube. For abelian varieties over finite fields it has been proved by Soulé [Sou1, 3.5] (cf. also [Kü2]).

\section{Mixed motivic sheaves}

Before we state Beilinson's conjecture in its most general form, we formulate an absolute version that already reveals a lot of the basic philosophy about mixed motives and their relation with Chow groups.

CONJECTURE 4.1 (Version 3 of Beilinson's conjecture). There exists a rigid abelian $\mathbb{Q}$-linear tensor category $\mathscr{M}_{k}$ (of mixed motives over $k$ ) and a contravariant functor

$$
R: \mathscr{V}_{k} \rightarrow D^{b}\left(\mathscr{M}_{k}\right),
$$

where $D^{b}\left(\mathscr{M} \mathscr{M}_{k}\right)$ is the derived category formed by bounded complexes in $\mathscr{M} \mathscr{M}_{k}$, such that the following hold.

(i) (Künneth formula) There are functorial quasi-isomorphisms

$$
R(X) \otimes^{L} R(Y) \rightarrow R(X \times Y),
$$

where $\otimes^{L}$ denotes the left derivative of $\otimes$, satisfying an obvious compatibility with the associativity and commutativity constraints of $\mathscr{M M}_{k}$.

(ii) (trace map) If $X$ is pure of dimension $d$, then there is a canonical morphism

$$
\eta_{X}: R(X) \rightarrow 1(-d)[-2 d],
$$

where $1(-d)=H^{2}\left(R\left(\mathbb{P}_{k}^{1}\right)\right)^{\otimes d} \in O b\left(\mathscr{M} \mathscr{M}_{k}\right)$. If $Y$ is pure of dimension $e$, and if we let $\mathrm{pt}=\mathrm{Spec} k$, then the following diagrams commute:

$$
\begin{aligned}
& R(X) \otimes^{L} R(Y) \quad \rightarrow \quad R(X \times Y) \quad R(\mathrm{pt}) \otimes^{L} R(X) \rightarrow R(X) \\
& \eta_{X} \otimes \eta_{Y} \downarrow \quad\left\lfloor\eta_{X \times Y} \quad \eta_{\mathrm{pt}} \otimes \mathrm{id} \downarrow \quad \|\right. \\
& 1\{-d\} \otimes 1\{-e\} \quad \rightarrow \quad 1\{-d-e\} \quad 1 \otimes R(X) \stackrel{\sim}{\rightarrow} R(X),
\end{aligned}
$$


where $1(0)=1$ is the neutral object of $\mathscr{M M}_{k}$, and where we put $1\{-d\}=$ $1(-d)[-2 d]$.

(iii) (Poincare duality) If $X$ is pure of dimension $d$, then the pairing

$$
R(X) \otimes \otimes^{L} R(X) \rightarrow R(X \times X) \stackrel{\Delta^{*}}{\rightarrow} R(X) \stackrel{\eta_{X}}{\rightarrow} 1(-d)[-2 d]
$$

induces a quasi-isomorphism

$$
R(X)(d)[2 d] \stackrel{\sim}{\rightarrow} R \underline{\operatorname{Hom}}(R(X), 1) .
$$

Here $R \underline{\mathrm{Hom}}$ is the right derivative of $\underline{\mathrm{Hom}}$, the internal hom in $\mathscr{M M}_{k}$, and for an object $C$ in $D^{b}\left(\mathscr{M M}_{k}\right)$ and $n \in \mathbb{Z}$ we put

$$
C(n)=C \otimes 1(n),
$$

where for $n \geq 0$ we let $1(n)=\underline{\operatorname{Hom}}(1(-n), 1)$, the dual of $1(-n)$.

(iv) (cycle map) There are functorial isomorphisms

$$
c l=c l_{X}^{j}: C H^{j}(X)_{\mathbb{Q}} \rightarrow \operatorname{Hom}_{\mathscr{D}}(1, R(X)(j)[2 j]),
$$

where we denote $\mathscr{D}=\mathscr{D}^{b}\left(\mathscr{M}_{k}\right)$ for short, such that the following compatiblities are satisfied:

(a) via $\mathrm{cl}$, the intersection pairing

$$
C H^{i}(X) \otimes C H^{j}(X) \rightarrow C H^{i+j}(X)
$$

is compatible with the pairing induced by

$$
R(X) \otimes^{L} R(X) \rightarrow R(X \times X) \stackrel{\Delta^{*}}{\rightarrow} R(X) ;
$$

(b) if $X$ and $Y$ are of pure dimensions $d$ and $e$, respectively, and $f: X \rightarrow$ $Y$ is a morphism, then via $\mathrm{cl}$, the push-forward

$$
f_{*}: C H^{d+i}(X) \rightarrow C H^{e+i}(Y)
$$

is compatible with the morphism

$$
f_{*}: R(X)(d)[2 d] \rightarrow R(Y)(e)[2 e]
$$

induced by $f$ via (4.1) and the functorality of $R(X)$.

(v) (relation with motivic cohomology) More generally, there are functorial isomorphisms for $i, j \in \mathbb{Z}$

$$
H_{\mathscr{M}}^{i}(X, \mathbb{Q}(j)) \stackrel{\sim}{\rightarrow} \operatorname{Hom}_{\mathscr{D}}(1, R(X)(j)[i]),
$$

where $H_{\mathscr{M}}^{i}(X, \mathbb{Q}(j))=K_{2 j-i}(X)^{(j)}$ is motivic cohomology as defined via algebraic $K$-theory [Be1, 2.2.1; Sou2; Gray], such that compatibilities analogous to (iv)(a), (b) hold.

(vi) (relation with Grothendieck motives) The contravariant functor

$$
X \mapsto \bigoplus_{i} H^{i}(R(X)) \in O b\left(\mathscr{M} \mathscr{M}_{k}\right)
$$


identifies the category $\mathscr{M}_{k}$ of Grothendieck motives with a full abelian tensor subcategory of $\mathscr{M M}_{k}$ such that

$$
H^{i}(R(X))=h^{i}(X) \in O b\left(\mathscr{M}_{k}\right)
$$

under this identification.

Remarks 4.2. (a) Since $\otimes$ and $\underline{\mathrm{Hom}}$ are exact functors for a rigid abelian tensor category (cf. [DM, 1.16]), they derive trivially. Hence for complexes $A^{*}, B^{*}, C^{*}$ in $\mathscr{M M}_{k}, A^{*} \otimes^{L} B^{\circ}$ is represented by $A^{*} \otimes B^{*}, R \underline{\operatorname{Hom}}\left(A^{*}, B^{*}\right)$ is represented by $\underline{\operatorname{Hom}}\left(A^{\circ}, B^{*}\right)$, and we have formulae like

$$
\operatorname{Hom}_{\mathscr{D}}\left(A^{\cdot}, R \underline{\operatorname{Hom}}\left(B^{\cdot}, C^{*}\right)\right) \cong \operatorname{Hom}_{\mathscr{D}}\left(A^{*} \otimes{ }^{L} B^{\cdot}, C^{*}\right) \text {. }
$$

In fact, $\otimes^{L}$ and $R \underline{\text { Hom }}$ give $D^{b}\left(\mathscr{M} \mathscr{M}_{k}\right)$ the structure of a rigid $\mathbb{Q}$-linear tensor category.

(b) By (a), every pairing $A \otimes^{L} B \rightarrow C$ induces a morphism $B \rightarrow$ $R \underline{\operatorname{Hom}}(A, C)$. This explains the morphism (4.1).

(c) It follows from the axioms that $\eta_{Y} f_{*}=\eta_{X}$ for $f: X \rightarrow Y$. One gets an equivalent set of axioms, if instead of (iv)(b) one postulates: if $X$ is of pure dimension $d$, then via $c l$, the homomorphism

$$
\left(p_{Y}\right)_{*}: C H^{j}(X \times Y) \rightarrow C H^{j-d}(Y)
$$

induced by the projection $p_{Y}: X \times Y \rightarrow Y$ is compatible with the composition

$$
R(X \times Y) \stackrel{(i)}{\sim} R(X) \otimes^{L} R(Y) \stackrel{\eta_{X} \otimes \text { id }}{\rightarrow} 1(-d)[-2 d] \otimes R(Y) .
$$

(d) The meaning of Conjcture 4.1(vi) is as follows. Let $X$ be of pure dimension $d$. Then we have canonical isomorphisms

$$
C H^{d}(X \times Y)_{\mathbb{Q}} \stackrel{\sim}{\rightarrow} \operatorname{Hom}_{\mathscr{D}}(R(X), R(Y))
$$

by composiing $c l_{X \times Y}^{d}$ with the isomorphisms

$$
\begin{aligned}
\operatorname{Hom}_{\mathscr{D}} & (1, R(X \times Y)(d)[2 d]) \\
& \cong \operatorname{Hom}_{\mathscr{D}}\left(1, R(X)(d)[2 d] \otimes^{L} R(Y)\right) \quad[\text { from (i)] } \\
& \cong \operatorname{Hom}_{\mathscr{D}}\left(1, R \underline{\operatorname{Hom}}(R(X), 1) \otimes^{L} R(Y)\right) \quad[\text { from (iii)] } \\
& \cong \operatorname{Hom}_{\mathscr{D}}(1, R \underline{\operatorname{Hom}}(R(X), R(Y))) \quad[\mathrm{cf} .[\mathrm{DM},(1.6 .10)]] \\
& \cong \operatorname{Hom}_{\mathscr{D}}(R(X), R(Y)) \quad[\mathrm{cf} .(\mathrm{a})] .
\end{aligned}
$$

Moreover, one checks that via these isomorphisms composition of correspondences corresponds to composition of morphisms in $\mathscr{D}$. Now Conjecture 4.1 (vi) means that by passing to the cohomology, (4.2) induces an isomorphism

$$
\begin{aligned}
& C H^{d}(X \times Y)_{\mathbb{Q}} / \sim_{\text {hom }} \stackrel{\sim}{\rightarrow} \underset{i}{\bigoplus} \operatorname{Hom}_{{\mathscr{M M _ { k }}}_{k}}\left(H^{i}(R(X)), H^{i}(R(Y))\right) \\
& \| \\
& \operatorname{Hom}_{\mathscr{M}_{k}}(h(X), h(Y))
\end{aligned}
$$


such that for $Y=X$ the central idempotent projecting to $\operatorname{End}_{M_{k}}\left(H^{i}(R(X))\right)$ corresponds to $\pi_{i}$, the $i$ th Künneth component of the diagonal (cf. (2.2)). It is easy to see that then the association

$$
h^{i}(X) \mapsto H^{i}(R(X)) \in O b\left(\mathscr{M} \mathscr{M}_{k}\right)
$$

induces a fully faithful embedding $\mathscr{M}_{k} \subset \mathscr{M} \mathscr{M}_{k}$ as wanted. In particular, this implies that the objects $1(n)$ are the usual Tate objects considered in $\mathscr{M}_{k}$.

(e) The complex $R(X)$ should be thought of as the motivic analogue of the complex $R \Gamma\left(\bar{X}, \mathbb{Q}_{\ell}\right)$ in the derived category $D^{b}\left(G_{k}, \mathbb{Q}_{\ell}\right)$ of $\mathbb{Q}_{\ell}-$ representations of $G_{k}$ computing $H^{*}\left(\bar{X}, \mathbb{Q}_{\ell}\right)$ or of the complex $R \Gamma(X(\mathbb{C}), \mathbb{Q})$ in $D^{b}(\mathbb{Q}-\mathrm{MHS})$ computing $H^{*}(X(\mathbb{C}), \mathbb{Q})$ with its Hodge structure (cf. [Be2]). In fact, a natural complement to Conjecture 4.1 is the assumption

(f) There exist exact faithful tensor functors $H_{\ell}$ and $H_{B}$ on $\mathscr{M M}_{k}$ as in (2.10) such that there are functorial quasi-isomorphisms

$$
\begin{aligned}
& H_{\ell} R(X) \simeq R \Gamma\left(\bar{X}, \mathbb{Q}_{\ell}\right) \quad(\ell \neq \text { char } k), \\
& H_{B} R(X) \simeq R \Gamma(X(\mathbb{C}), \mathbb{Q}) \quad(k=\mathbb{C}),
\end{aligned}
$$

compatible with the structures in Conjecture 4.1(i)-(v).

Lemma 4.3. If $X \mapsto R(X)$ is a functor satisfying Conjecture 4.1(i)-(iv), (vi), then one has a (noncanonical) quasi-isomorphism

$$
R(X) \stackrel{\sim}{\rightarrow} \bigoplus_{i \geq 0} h^{i}(X)[-i]
$$

Proof (cf. [De1, 1.11]). Let $\pi_{j} \in C H^{d}(X \times X)_{\mathbb{Q}}(d=\operatorname{dim} X)$ also denote some lifts of the Künneth components of the diagonal $(j=0, \ldots, 2 d)$. Via (4.2), each $\pi_{j}$ can be regarded as an endomorphism of $R(X)$, and by (4.3) and the identification $H^{i}(R(X))=h^{i}(X)$, the induced endomorphism on $H^{i}(R(X))$ is $\delta_{i, j} \cdot$ id. Since the Ext spectral sequence

$$
E_{2}^{p, q}=\operatorname{Ext}_{\text {}^{p} \boldsymbol{\mu}_{k}}\left(H^{i}(R(X)), H^{q}(R(X))\right) \Rightarrow \operatorname{Hom}_{\mathscr{D}}\left(H^{i}(R(X)), R(X)[p+q]\right)
$$

is functorial with respect to the endomorphisms $\pi_{j}$ of $R(X)$, we immediately conclude that it degenerates. Hence the edge morphism

$$
\begin{aligned}
& \operatorname{Hom}_{\mathscr{D}}\left(H^{i}(R(X)), R(X)[i]\right) \rightarrow \operatorname{Hom}_{\mathscr{M A}_{k}}\left(H^{i}(R(X)), H^{i}(R(X))\right), \\
& f \mapsto H^{i}(f)
\end{aligned}
$$

is surjective. Thus, for all $i$ there are $a_{i}: H^{i}(R(X))[-i] \rightarrow R(X)$ inducing the identity on the $i$ th cohomology. Then

$$
\sum a_{i}: \bigoplus_{i} H^{i}(R(X))[-i] \rightarrow R(X)
$$

is a quasi-isomorphism. 
Proposition 4.4. Version 3 implies version 2 of Beilinson's conjecture.

Proof. Let $F^{\cdot}$ with $F^{\nu} / F^{\nu+1}=E_{\infty}^{\nu, 2 j-\nu}$ be the descending filtration on

$$
C H^{j}(X)_{\mathbb{Q}} \stackrel{\sim}{\rightarrow} \operatorname{Hom}_{\mathscr{D}}(1, R(X)(j)[2 j])
$$

given by the Ext spectral sequence

$$
E_{2}^{p, q}=\operatorname{Hom}_{\mathscr{D}}\left(1, H^{q}(R(X)(j))[p]\right) \Rightarrow \operatorname{Hom}_{\mathscr{D}}(1, R(X)(j)[p+q]) .
$$

By Lemma 4.3 (or by the argument used in its proof) this spectral sequence degenerates, and we get

$$
\operatorname{Gr}_{F}^{\nu} C H^{j}(X)_{\mathbb{Q}} \cong E_{2}^{\nu, 2 j-\nu}=\operatorname{Ext}_{\mathscr{M K}_{k}}^{\nu}\left(1, h^{2 j-\nu}(X)(j)\right),
$$

i.e., Beilinson's formula. In fact, for an abelian category $\mathscr{A}$ and objects $A, B$ in $\mathscr{A}$ one has

$$
\operatorname{Hom}_{D^{b}(\mathscr{A})}(A, B[m])= \begin{cases}0, & m<0, \\ \operatorname{Ext}_{\mathscr{A}}^{m}(A, B), & m \geq 0,\end{cases}
$$

where $A$ and $B$ are regarded as complexes concentrated in degree zero and $\operatorname{Ext}_{\mathscr{Q}}^{m}$ denotes the Yoneda-Ext groups [Ver, 2.3]. Since $R(X)$ is a bounded complex, the spectral sequence converges, and we have $F^{0}=C H^{j}(X)_{\mathbb{Q}}$ and $F^{\nu}=0$ for $\nu \gg 0$, i.e., property (e) of version 2 . The rest of the properties (a)-(c) and $\left(\mathrm{d}^{\prime}\right)$ easily follows from (i)-(iv) and (vi) of version 3. Indeed, the equality $F^{1}=C H^{j}(X)_{\text {hom, } Q}$ is a special case of (vi): we have

$$
\begin{aligned}
\operatorname{Gr}_{F}^{0} C H^{j}(X)_{\mathbb{Q}} & =E_{2}^{0,2 j}=\operatorname{Hom}_{\mathscr{M M}_{k}}\left(1, h^{2 j}(X)(j)\right) \\
& =\operatorname{Hom}_{\mathscr{M}_{k}}\left(1, h^{2 j}(X)(j)\right)=C H^{j}(X)_{\mathbb{Q}} / \sim_{\text {hom }} ;
\end{aligned}
$$

cf. (4.4). For the functoriality in (b), (c), and $\left(d^{\prime}\right)$ note that by definition the Ext spectral sequence (4.6) is induced by the canonical filtration $\tau_{\leq}$, which is respected by all morphisms in the derived cateogry. Hence (4.6) and consequently $F^{\circ}$ is functorial for morphisms in $\mathscr{D}$ (this was already used in the proof of 4.3). Since (4.5) is functorial, we obtain that $F^{*}$ is respected by $f^{*}$ for morphisms $f: X \rightarrow Y$. More generally, via (4.5) the action of correspondences $\alpha$ on $\mathrm{CH}^{*}()$ corresponds to the map obtained on $\operatorname{Hom}_{\mathscr{D}}(1, R(-)(-)[-])$ by functoriality from the morphism $\mathrm{cl}(\alpha)$ in $\mathscr{D}$ associated to $\alpha$ via (4.3) - this is a special case of the compatibility of (4.3) with composition. In particular, $F^{*}$ is also respected by $f_{*}$, showing (c), and (4.7) is functorial for correspondences, completing $\left(\mathrm{d}^{\prime}\right)$.

For 2.1(b) we note that by our definition we have

$$
\begin{aligned}
F^{\nu} C H^{j}(X)_{\mathbb{Q}}=\operatorname{Im}( & \operatorname{Hom}_{\mathscr{D}}\left(1, \tau_{\leq-\nu}(R(X)(j)[2 j])\right) \\
& \left.\rightarrow \operatorname{Hom}_{\mathscr{D}}(1, R(X)(j)[2 j])\right) .
\end{aligned}
$$

But

$$
\tau_{\leq-r}(R(X)(i)[2 i]) \otimes{ }^{L} \tau_{\leq-s}(R(Y)(j)[2 j])
$$


maps to $\tau_{\leq-r-s}\left(R(X) \otimes^{L} R(Y)(i+j)[2 i+2 j]\right)$ and hence to

$$
\tau_{\leq-r-s}(R(X \times Y)(i+j)[2(i+j)])
$$

under the quasi-isomorphism (i) (quasi-isomorphically, since $\otimes$ is exact). By the compatibility between intersection product and the pairing on $R(X)$ stated in (iv)(a), we get (b) of version 2 .

REMARKS 4.5. (a) A more concrete description is obtained by the decomposition in 4.3. Via this isomorphism,

$$
\tau_{\leq m} R(X) \simeq \bigoplus_{i=0}^{m} h^{i}(X)[-i] .
$$

Note that the decomposition is not unique whereas the filtration is. But the decomposition implies that the map in (4.9) is injective and that we have noncanonical isomorphisms

$$
\begin{aligned}
\operatorname{Hom}_{\mathscr{D}}(1, R(X)(j)[2 j]) & \cong \bigoplus_{i \geq 0} \operatorname{Hom}_{\mathscr{D}}\left(1, h^{i}(X)(j)[2 j-i]\right) \\
& =\bigoplus_{i=0}^{2 j} \operatorname{Ext}_{\mathscr{M} \mathscr{K}_{k}}^{2 j-i}\left(1, h^{i}(X)(j)\right)
\end{aligned}
$$

under which

$$
F^{\nu} \operatorname{Hom}_{\mathscr{D}}(1, R(X)(j)[2 j]) \cong \bigoplus_{i=0}^{2 j-\nu} \operatorname{Ext}_{\mathscr{M K _ { k }}}^{2 j-i}\left(1, h^{i}(X)(j)\right) .
$$

(b) Part (v) of version 3 would imply similar filtrations $F^{\circ}$ on motivic cohomology $H_{\mathscr{M}}^{i}(X, \mathbb{Q}(j))$. We would get

(ã) $F^{0} H_{\mathscr{M}}^{i}(X, \mathbb{Q}(j))=H_{\mathscr{M}}^{i}(X, \mathbb{Q}(j)), F^{1} C H^{j}(X)_{\mathbb{Q}}=C H^{j}(X)_{\mathrm{hom}, \mathbb{Q}}$.

(i) $F^{r} H_{\mathscr{M}}^{i_{1}}\left(X, \mathbb{Q}\left(j_{1}\right)\right) \cdot F^{s} H_{\mathscr{M}}^{i_{2}}\left(X, \mathbb{Q}\left(j_{2}\right)\right) \subset F^{r+s} H_{\mathscr{K}}^{i_{1}+i_{2}}\left(X, \mathbb{Q}\left(j_{1}+j_{2}\right)\right)$ for the product in motivic cohomology.

(i) $F$ is respected by $f^{*}$ and $f_{*}$ for morphisms $f: X \rightarrow Y$.

(d) There are functorial isomorphisms

$$
\operatorname{Gr}_{F}^{\nu} H_{\mathscr{M}}^{i}(X, \mathbb{Q}(j))=\operatorname{Ext}_{\mathscr{M K}_{k}}^{\nu}\left(1, h^{i-\nu}(X)(j)\right),
$$

in particular, $\operatorname{Gr}_{F}^{\nu} H_{\mathscr{M}}^{i}(X, \mathbb{Q}(j))$ only depends on $h^{i-\nu}(X)$, as a functor on $\mathscr{M}_{k}$.

(ẽ) $F^{\nu} H_{\mathscr{M}}^{i}(X, \mathbb{Q}(j))=0$ for $\nu \gg 0$.

Denote by $\mathscr{C} \mathscr{M} \mathscr{M}_{k}=\mathscr{M}_{k}^{\text {rat }}$ the category of motives modulo rational equivalence; i.e., we take the whole Chow groups for the correspondences. We sometimes call the objects in $\mathscr{C} \mathscr{M} \mathscr{M}_{k}$ Chow motives.

LEMMA 4.6. If $X \mapsto R(X)$ is a functor satisfying 4.1(i)-(iv), (vi), it induces a fully faithful embedding of $\mathbb{Q}$-linear tensor categories

$$
i: \mathscr{C O H M}_{k} \hookrightarrow D^{b}\left(\mathscr{M} \mathscr{M}_{k}\right) \text {. }
$$


Proof. Let $\operatorname{ch}(X)=(X, \text { id }, 0)_{\text {rat }}$ be the Chow motive associated to $X \in$ $O b\left(\mathscr{V}_{k}\right)$. Then the formula (4.2) means that we have the embedding $i$ on the full subcategory of $\mathscr{C} \mathscr{K M}{ }_{k}$ formed by all $\operatorname{ch}(X)$, by putting $i(\operatorname{ch}(X))=$ $R(X)$. By the definitions, this embedding is compatible with tensor products, and the canonical decomposition $\operatorname{ch}\left(\mathbb{P}_{k}^{1}\right)=1 \oplus 1(-1)$ carries over to the decomposition $R\left(\mathbb{P}_{k}^{1}\right)=1 \oplus 1(-1)[-2]$. Hence we have to show that for a projector $p=p^{2} \in \operatorname{End}(R(X))$ the kernels of $p$ and $1-p$ exist and that the canonical morphisms induce an isomorphism

$$
\operatorname{Ker} p \oplus \operatorname{Ker}(1-p) \stackrel{\sim}{\rightarrow} R(X) .
$$

Consider the decomposition

$$
R(X) \cong \bigoplus_{i} h^{i}(X)[-i]
$$

of Lemma 4.3. Recall (cf. (4.8)) that we have

$$
\begin{aligned}
\operatorname{Hom}_{\mathscr{D}} & \left(h^{i}(X)[-i], h^{j}(X)[-j]\right) \\
& \cong \operatorname{Hom}_{\mathscr{D}}\left(h^{i}(X), h^{j}(X)[i-j]\right)=0 \quad \text { for } i<j .
\end{aligned}
$$

This shows that the kernel $J$ of the epimorphism

$$
\operatorname{End}_{\mathscr{D}}(R(X)) \rightarrow \bigoplus_{i} \operatorname{End}_{\mathscr{M}_{k}}\left(h^{i}(X)\right)
$$

is a nilpotent ideal. Hence every projector $p \in \operatorname{End}(R(X))$ can be written as

$$
p=(1-\eta) p^{\prime}(1-\eta)^{-1},
$$

where $p^{\prime}$ is "diagonal" with respect to the decomposition (4.12) and $\eta \in J$ (cf. Lemma 5.4). Obviously, $\operatorname{Ker} p^{\prime}$ and $\operatorname{Ker}\left(1-p^{\prime}\right)$ exist, and the decomposition (4.11) holds for $p^{\prime}$, since this is true for its components in End $_{\mathscr{D}}\left(h^{i}(X)[-i]\right) \cong$ End $_{\mathscr{M}_{k}}\left(h^{i}(X)\right)$. But then (4.13) implies the claim for $p$.

4.7. As Deligne points out (cf. [De4, §3]), it might be too optimistic to hope for a description inside a category $D^{b}\left(\mathscr{M M}_{k}\right)$ as above. A weaker and more cautious conjecture would result after the following changes:

(1) Replace $D^{b}\left(\mathscr{M} \mathscr{M}_{k}\right)$ by a general triangulated category $\mathscr{D}$ with a $t$ structure $\left(\mathscr{D}^{\geq 0}, \mathscr{D}^{\leq 0}\right)$ in the sense of [BBD, 1.3].

(2) Replace $H^{i}(-)$ by the cohomological functors ${ }^{t} H^{i}(-)={ }^{t} \tau_{\leq i}{ }^{t} \tau_{\geq i}$ associated to the $t$-structure [BBD, 1.3.6].

(3) Replace $\otimes^{L}$ by some tensor law $\otimes$ on $\mathscr{D}$ that is compatible with the triangulation and $t$-structure and $R \underline{\mathrm{Hom}}$ by an internal hom associated to $\otimes(\mathscr{D}$ should be rigid).

(4) $\mathscr{M} \mathscr{M}_{k}$ would then be the heart $\mathscr{D}^{\leq 0} \cap \mathscr{D}^{\geq 0}$ of the $t$-structure, which is an abelian category (loc. cit.). 
The point here is that $\mathscr{D}$ is not necessarily equivalent to $D^{b}\left(\mathscr{M M}_{k}\right)$. The analogues of Lemmas 4.3 and 4.6 would hold, and the theory of exact couples would still give a degenerate spectral sequence

$$
E_{2}^{p, q}=\operatorname{Hom}_{\mathscr{D}}\left(1,{ }^{t} H^{q}(R(X)(j)[p])\right) \Rightarrow \operatorname{Hom}_{\mathscr{D}}(1, R(X)(j)[p+q]) .
$$

This would still give a filtration on the limit terms $\operatorname{Hom}_{\mathscr{D}}(1, R(X)(j)[i]) \cong$ $H_{\mathscr{M}}^{i}(X, \mathbb{Q}(j))$ with all the properties in version 1 of Beilinson's conjecture, but for the successive quotients we would get

$$
\operatorname{Gr}_{F}^{\nu} C H^{j}(X)_{\mathbb{Q}}=\operatorname{Hom}_{\mathscr{D}}\left(1, h^{2 j-\nu}(X)(j)[\nu]\right),
$$

which is not necessarily isomorphic to

$$
\operatorname{Ext}_{\mathscr{M M}_{k}}^{\nu}\left(1, h^{2 j-\nu}(X)(j)\right)=\operatorname{Hom}_{D^{b}\left(\mathscr{M}_{k}\right)}\left(1, h^{2 j-\nu}(X)(j)[\nu]\right)
$$

(cf. [BBD, 3.1.6]).

This caveat is incorporated in the following broadest version of Beilinson's conjecture. Since it mainly serves as a philosophical background for more precise conjectures, and it would be somewhat difficult to express all data and compatibilities precisely, we only state it in a vague form and then add some discussion.

Conjecture 4.8 (Version 4 of Beilinson's conjecture; cf. [Be3, 5.10]). For every algebraic $k$-scheme $X$ there is a triangulated $\mathbb{Q}$-linear tensor category $\mathscr{D} \mathscr{M}(X)$ with a $t$-structure such that the following hold.

(1) There are the usual six functors

$$
f_{*}, f_{!}, f^{*}, f^{!}, \otimes, \underline{\text { Hom }}
$$

between these categories, i.e.,

(a) On each $\mathscr{D} \mathscr{M}(X)$ there is a tensor law $\otimes$ and an associated internal hom Hom;

(b) If $f: X \rightarrow Y$ is a morphism, there are functor

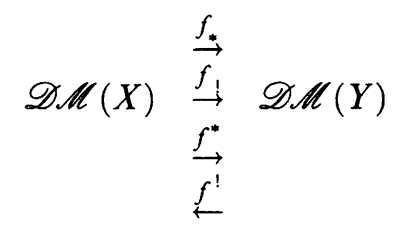

satisfying the usual properties of Grothendieck-Verdier duality theory; e.g., $f^{*}$ is left adjoint to $f_{*}, f_{!}$is left adjoint to $f^{!}$, there is a projection formula for $f_{*}$ and $f^{*}$, and one for $f^{!}$and $f_{!}$, the operation $f \mapsto f_{*}$ is functorial, and so forth.

(2) Let $\mathscr{M}(X)$ (the category of mixed motivic sheaves on $X$ ) be the abelian $\mathbb{Q}$-linear category which is the heart of the t-structure on $\mathscr{D} \mathscr{M}(X)$. Then there is a weight filtration on $\mathscr{M}(X)$; i.e., the objects $M$ in $\mathscr{M}(X)$ have functorially associated increasing "weight" filtrations W.M such that $M \mapsto$ 
$W_{m} M$ is an exact functor for every $m \in \mathbb{Z}$. In addition, the objects $\mathrm{Gr}_{m}^{W} M$ are semisimple.

(3) There are canonical ("Tate") objects $\mathbb{Q}_{\mathscr{M}}(i)=\mathbb{Q}_{\mathscr{M}, X}(i)$ in $\mathscr{D} \mathscr{M}(X)$, for $i \in \mathbb{Z}$, together with compatible isomorphisms $\mathbb{Q}_{\mathscr{M}}(i) \otimes \mathbb{Q}_{\mathscr{M}}(j) \stackrel{\sim}{\rightarrow} \mathbb{Q}_{\mathscr{M}}(i+j)$.

(4) The subcategory of semisimple objects in $\mathscr{M}(\operatorname{Spec} k)$ is equivalent to the category $\mathscr{M}_{k}$ of Grothendieck motives over $k$. Under this equivalence, $\mathbb{Q}_{\mathscr{M}, \mathrm{Spec} k}(j)$ corresponds to the Tate object $1(j)$, and for a smooth projective variety $a: X \rightarrow \operatorname{Spec} k$ the object $H^{i}\left(a_{*} \mathbb{Q}_{\mathscr{M}, X}\right)$ corresponds to $h^{i}(X) \in$ $O b\left(\mathscr{M}_{k}\right)$ (here $H^{i}={ }^{t} H^{i}$ is the ith cohomological functor associated to the $t$-structure on $\mathscr{D} \mathscr{M}(\operatorname{Spec} k))$.

(5) There are canonical homomorphisms

$$
H_{\mathscr{M}}^{i}(X, \mathbb{Q}(j)) \rightarrow \operatorname{Hom}_{\mathscr{D M}(X)}\left(\mathbb{Q}_{\mathscr{M}}, \mathbb{Q}_{\mathscr{M}}(j)[i]\right)
$$

that are isomorphisms for regular $X$.

(6) There are exact ("realization") functors

$$
\begin{array}{ll}
r_{\ell}: \mathscr{D} \mathscr{M}(X) \rightarrow D_{m}^{b}\left(X_{\text {ét }}, \mathbb{Q}_{\ell}\right), & \ell \neq \operatorname{char}(k), \\
r_{B}: \mathscr{D M}(X) \rightarrow D^{b}(\mathscr{M} \mathscr{H}(X)), \quad k=\mathbb{C},
\end{array}
$$

where $D_{m}^{b}\left(X_{\hat{\mathrm{et}}}, \mathbb{Q}_{\ell}\right)$ is the derived category of bounded complexes of étale $\mathbb{Q}_{\ell}$ sheaves on $X$ with mixed constructible cohomology [BBD, 5.15] and $\mathscr{M} \mathscr{C}(X)$ is the category of mixed Hodge modules on $X$ [SaM1, 2.17]. These functors are compatible with the above structures and map $\mathscr{M}(X)$ to the categories $\mathscr{M} \mathscr{P}\left(X_{\text {et }}, \mathbb{Q}_{\ell}\right)$ of mixed perverse étale $\mathbb{Q}_{\ell}$-sheaves on $X$ [BBD, 5.1.7] and to $\mathscr{M} \mathscr{C}(X)$, respectively. Furthermore, they are exact and faithful on $\mathscr{M}(X)$.

4.9. Let us add some explanation. The existence of the homomorphisms in (5) should follow from the data in (1) and (3), perhaps together with some extra assumptions. Namely, as for the $\ell$-adic cohomology, the "duality theory" of (1) should imply that the associations

$$
\begin{aligned}
(Z \stackrel{i}{\hookrightarrow} X) & \mapsto H_{Z}^{i}(X, j)=\operatorname{Hom}_{\mathscr{D M}(X)}\left(\mathbb{Q}_{\mathscr{M}}, i_{*} i \mathbb{Q}_{\mathscr{M}}(j)[i]\right), \\
(X \stackrel{f}{\rightarrow} \operatorname{Spec} k) & \mapsto H_{a}(X, b)=\operatorname{Hom}_{\mathscr{D M}(X)}\left(\mathbb{Q}_{\mathscr{M}}, f^{\prime} \mathbb{Q}_{\mathscr{M}}(-a)[-b]\right)
\end{aligned}
$$

define a twisted Poincaré duality theory in the sense of Bloch and Ogus [BO, $\S 1$. Some additional properties (as in [Bei1, 2.3] or in [Gi, 1.1 and 1.2]) should give canonical Chern character maps

$$
K_{2 j-i}(X) \rightarrow H^{i}(X, j)
$$

inducing the maps in (5).

As for (6), the categories $D_{m}^{b}\left(X, \mathbb{Q}_{\ell}\right)$ and $\mathscr{M P}\left(X, \mathbb{Q}_{\ell}\right)$ have only been defined under some finiteness conditions in [BBD], but a more sophisticated theory of $\mathbb{Q}_{\ell}$-sheaves (cf., e.g., [Ek]) should allow defining them in general. Concerning the analogy between $r_{\ell}$ and $r_{B}$, we note that Beilinson 
has proved remarkable equivalences of categories (cf. [Be4, 1.3]),

$$
\begin{gathered}
D^{b}\left(\mathscr{M P}\left(X_{\text {ét }}, \mathbb{Q}_{\ell}\right)\right) \stackrel{\text { real }}{\sim} D_{m}^{b}\left(X_{\text {ét }}, \mathbb{Q}_{\ell}\right), \quad \ell \neq \text { char } k, \\
D^{b}(\mathscr{P}(X, \mathbb{Q})) \stackrel{\text { real }}{\sim} D_{c}^{b}(X(\mathbb{Q}), \mathbb{C}), \quad k=\mathbb{C},
\end{gathered}
$$

where $D_{c}^{b}(X(\mathbb{C}), \mathbb{Q})$ is the derived category of bounded complexes of $\mathbb{Q}$ sheaves on $X(\mathbb{C})$ (with the strong topology) with algebraically constructible cohomology and $\mathscr{P}(X, \mathbb{Q}) \subset D_{c}^{b}(X(\mathbb{C}), \mathbb{Q})$ is the category of algebraically constructible perverse $\mathbb{Q}$-sheaves on $X(\mathbb{C})$. On the other hand, one has a forgetful functor [SaM1]

$$
\mathscr{M} \mathscr{H}(X) \rightarrow \mathscr{P}(X, \mathbb{Q}) .
$$

The compatibility of $r_{\ell}$ or $r_{B}$ with the structures in (1)-(5) means, e.g., the following: $r_{\ell}$ "commutes" with the six functors which also exist on $D_{c}^{b}\left(-, \mathbb{Q}_{\ell}\right)$. For example, one should have $r_{\ell} f_{*}=f_{*} r_{\ell}$, where we also write $f_{*}$ for what is often denoted $R f_{*}: D_{m}^{b}\left(X_{\text {et }}, \mathbb{Q}_{\ell}\right) \rightarrow D_{m}^{b}\left(Y_{\text {et }}, \mathbb{Q}_{\ell}\right)$. Furthermore, $\mathbb{Q}_{\mathscr{M}}(i)$ should map to the sheaf $\mathbb{Q}_{\ell}(i)$ on $X$, and the weight filtration should map to the weight filtration in $\mathscr{M} \mathscr{P}\left(X_{\text {ét }}, \mathbb{Q}_{\ell}\right)$ via $r_{\ell}$. Similar facts should hold for $r_{B}$. Note that $r_{\ell}$ and $r_{B}$ would induce maps

$$
\operatorname{Hom}_{\mathscr{D M}(X)}\left(\mathbb{Q}_{\mathscr{K}}, \mathbb{Q}_{\mathscr{M}}(j)[i]\right)
$$

$$
\operatorname{Hom}_{D_{m}^{b}\left(X_{\text {ét }}, \mathbb{Q}_{\ell}\right)}\left(\mathbb{Q}_{\ell}, \mathbb{Q}_{\ell}(j)[i]\right)=H^{i}\left(X_{\text {ét }}, \mathbb{Q}_{\ell}(j)\right)
$$

$$
\operatorname{Hom}_{D^{b}(\mathscr{M P}(X))}(\mathbb{Q}, \mathbb{Q}(j)[i])=H_{\mathscr{P}}^{i}(X, \mathbb{Q}(j))
$$

into continuous étale and absolute Hodge cohomology (cf. [Be2]; $H_{\mathscr{H}}^{i}(X, \mathbb{Q}(j))$ coincides with Deligne cohomology $H_{\mathscr{D}}^{i}(X, \mathbb{Q}(j))$ if $\left.i \leq 2 j\right)$, respectively. The compositions with the maps in (5) should be the usual Chern character maps.

4.10. According to Beilinson's philosophy, the category $\mathscr{M}(X)$ of mixed motivic sheaves should correspond to the categories of mixed perverse sheaves in the realizations. For $X=\operatorname{Spec} k$, one has (4.18)

$$
\begin{aligned}
\mathscr{M P}\left((\operatorname{Spec} k)_{\text {ét }}, \mathbb{Q}_{\ell}\right) & =\text { mixed constructible } \mathbb{Q}_{\ell} \text {-sheaves on }(\operatorname{Spec} k)_{\text {ét }} \\
& =\text { mixed finite-dimensional } \mathbb{Q}_{\ell} \text {-representations of } G_{k} \\
\mathscr{M} \mathscr{C}(\operatorname{Spec} \mathbb{C}) & =\text { MHS. }
\end{aligned}
$$

In general, $\mathscr{M} \mathscr{P}\left(X_{\text {ét }}, \mathbb{Q}_{\ell}\right)$ is different from the category of mixed constructible $\mathbb{Q}_{\ell}$-sheaves on $X_{\mathrm{et}}$-and is better behaved than the latter; for example, it is an artinian category, and one has the decomposition theorem [BBD, 4.3.1 and 6.2.5]. 
4.11. One would deduce version 3 of Beilinson's conjecture from this version 4 as follows. Put

$$
\mathscr{M} \mathscr{M}_{k}=\mathscr{M}(\operatorname{Spec} k)
$$

(cf. the analogues (4.18)) and

$$
R(X)=a_{*} \mathbb{Q}_{\mathscr{M}, X} \in O b(\mathscr{D} \mathscr{M}(\operatorname{Spec} k))
$$

for $a: X \rightarrow$ Spec $k$ smooth and projective (recall that this corresponds to $R a_{*} \mathbb{Q}_{\mathscr{M}, X}$ in more traditional notation). The analogy with the realizations suggests that we should have

$$
\mathbb{Q}_{\mathscr{M}, X}=a^{*} \mathbb{Q}_{\mathscr{M}, \operatorname{Spec} k}
$$

This would imply isomorphisms

$$
\begin{aligned}
H_{\mathscr{M}}^{i}(X, \mathbb{Q}(j)) & \cong \operatorname{Hom}_{\mathscr{D} \mathscr{M}(X)}\left(a^{*} \mathbb{Q}_{\mathscr{M}}, a^{*} \mathbb{Q}_{\mathscr{M}}(j)[i]\right) \\
& \cong \operatorname{Hom}_{\mathscr{D} \mathscr{M}(\operatorname{Spec} k)}\left(\mathbb{Q}_{\mathscr{M}}, a_{*} a^{*} \mathbb{Q}_{\mathscr{M}}(j)[i]\right) \\
& =\operatorname{Hom}_{\mathscr{D} \mathscr{M}(\operatorname{Spec} k)}(1, R(X)(j)[i]),
\end{aligned}
$$

by (5), adjunction, and (4), hence the isomorphisms in (iv) and (v) of version 3 , as modified in 4.7. Their compatibility with product and push-forward (as in 4.7(iv)(a), (b)) would be a consequence of a Riemann-Roch theorem for the maps (4.14) as in [Bei1, 2.3.3] or [Gi, 4.1], if these are constructed as indicated above.

As for properties (i)-(iii) of version 3, we note that the corresponding statements hold for the $\ell$-adic analogues $R a_{*} \mathbb{Q}_{\ell}$ of the $R(X)$. Hence the properties for $R(X)$ can be seen as a consequence of the principle that the motivic theory "behaves the same way" as the theory of mixed $\ell$-adic sheaves [Bei3, 5.10, A]. We can deduce Conjecture 4.1(i)-(iii) in a more precise way from (6), viz., the assumption that $r_{\ell}$ is faithful on $\mathscr{M}(X)$. This assumption has the following two consequences. First, an object $C$ in $\mathscr{M}(X)$ is zero, if $r_{\ell}(C)$ is (look at the identity of $C$ ). Second, a morphism $\varphi$ in $\mathscr{D} \mathscr{M}(X)$ is an isomorphism, if $r_{\ell}(\varphi)$ is (apply the first property to the cohomology of $C$ in an exact triangle $A \stackrel{\varphi}{\rightarrow} B \rightarrow C \rightarrow A[1]$ in $\mathscr{D} \mathscr{M}(X))$.

Conjecture 4.1(i) can now be obtained as follows. The Cartesian diagram

$$
\begin{array}{ccc}
X \times Y & \stackrel{p_{2}}{\rightarrow} & Y \\
p_{1} \downarrow & & \downarrow b \\
X & \stackrel{a}{\rightarrow} & \text { Speck }
\end{array}
$$

induces a morphism in $\mathscr{D} \mathscr{M}(\operatorname{Spec} k)$

$$
\begin{aligned}
a_{*} \mathbb{Q}_{\mathscr{M}} \otimes b_{*} \mathbb{Q}_{\mathscr{M}} & \stackrel{\sim}{\rightarrow} a_{*}\left(\mathbb{Q}_{\mathscr{M}} \otimes a^{*} b_{*} \mathbb{Q}_{\mathscr{M}}\right) & & \text { [projection formula] } \\
& \rightarrow a_{*}\left(\mathbb{Q}_{\mathscr{M}} \otimes\left(p_{1}\right)_{*} p_{2}^{*} \mathbb{Q}_{\mathscr{M}}\right) & & {[\text { base change] }} \\
& \stackrel{\sim}{\rightarrow}\left(a p_{1}\right)_{*}\left(p_{1}^{*} \mathbb{Q}_{\mathscr{M}} \otimes p_{2}^{*} \mathbb{Q}_{\mathscr{M}}\right) & & {[\text { projection formula] }} \\
& \stackrel{\sim}{\rightarrow}\left(a_{X \times Y}\right)_{*} \mathbb{Q}_{\mathscr{M}} & & {\left[a_{X \times Y}=a p_{1}\right], }
\end{aligned}
$$


since $p_{1}^{*} \mathbb{Q}_{\mathscr{M}, X} \otimes p_{2}^{*} \mathbb{Q}_{\mathscr{M}, Y} \cong \mathbb{Q}_{\mathscr{M}, X \times Y} \otimes \mathbb{Q}_{\mathscr{M}, X \times Y} \cong \mathbb{Q}_{\mathscr{M}, X \times Y}$ by (4.21) and (3). Hence we have a morphism

$$
R(X) \otimes R(Y) \rightarrow R(X \times Y),
$$

which is a quasi isomorphism for smooth and proper $X$ and $Y$, since it is so after applying $r_{\ell}$, by the $\ell$-adic Künneth formula (cf. [Mi, VI, p. 261]). Similarly, we could have noted that the base change morphism

$$
a^{*} b_{*} \mathbb{Q}_{\mathscr{M}} \rightarrow\left(p_{1}\right)_{*}\left(p_{2}\right)^{*} \mathbb{Q}_{\mathscr{M}},
$$

obtained from the usual duality formalism, is a quasi-isomorphism, since its $\ell$-adic image is.

Concerning Conjecture 4.1(ii), the trace map $\eta_{X}: R(X) \rightarrow 1(-d)[-2 d]$ for $X$ smooth projective of dimension $d$ is obtained from the fact that

$$
H^{i}\left(a_{*} \mathbb{Q}_{\mathscr{M}}\right) \cong h^{i}(X)= \begin{cases}0, & i>2 d, \\ 1(-d), & i=2 d .\end{cases}
$$

The quasi-isomorphy in Conjecture 4.1(iii) follows as before, since it holds for the image under $r_{\ell}$ by the $\ell$-adic Poincare duality. Finally, property (vi) of version 3 is a consequence of property (4) of version 4.

Remarks 4.12. (a) Let $X$ be smooth and projective. The assumption on $r_{\ell}$ does not imply that $r_{\ell}$ is faithful on the triangulated category $\mathscr{D} \mathscr{M}(X)$, and this cannot be expected. In fact, the related map (cf. (4.17))

$$
H_{\mathscr{M}}^{i}(X, \mathbb{Q}(j)) \rightarrow H^{i}\left(X_{\text {ét }}, \mathbb{Q}_{\ell}(j)\right)
$$

will not in general be injective, as remarked after Lemma 2.7. The same is true for $r_{B}$, for example, by Mumford's counterexample recalled in $\S 1$. However, one may hope that $r_{\ell}$ is faithful on $\mathscr{D} \mathscr{M}(X)$ if $k$ is a finitely generated field; cf. Question 2.8.

(b) Beilinson hopes that the motivic theory of Conjecture 4.8 exists for arbitrary schemes (instead of just schemes of finite type over a field) and more general coefficients (instead of $\mathbb{Q}$-coefficients as above); cf. [Bei3, 5.10].

(c) For a finitely generated field $k$ of Kronecker dimension $r$ (= Krull dimension of an integral $\mathbb{Z}$-algebra of finite type with field of fractions $k$ ) one expects that the cohomological dimension of $\mathscr{M} \mathscr{M}_{k}=\mathscr{M}(\operatorname{Spec} k)$ is $r$, i.e., that $\mathrm{Ext}_{\mu_{\mu_{k}}}=0$ for $\nu>r$. We discuss two examples.

(i) $k=\mathbb{F}_{q}$, a finite field $(r=0)$. Here one expects that $\mathscr{M}_{k}=\mathscr{M}_{k}$, i.e., that every mixed motive is a direct sum of pure motives, and for a smooth variety $X / k$ one expects that the map

$$
H_{\mathscr{M}}^{i}(X, \mathbb{Q}(j)) \rightarrow H^{i}\left(\bar{X}, \mathbb{Q}_{\ell}(j)\right) \quad(\ell \neq \text { char } k)
$$

is injective. In fact, if $\operatorname{Ext}_{\mathscr{M} \mathscr{K}_{k}}^{\nu}=0$ for $\nu>0$, then

$$
\begin{aligned}
& \operatorname{Hom}_{D^{b}\left(\mu_{k}\right)}(1, R(X)(j)[i]) \\
& \cong \operatorname{Hom}_{\mathscr{M K}_{k}}\left(1, H^{i}(R(X)(j))\right) \\
& \stackrel{(6)}{\rightarrow} \operatorname{Hom}_{G_{k}}\left(\mathbb{Q}_{\ell}, H^{i}\left(\bar{X}, \mathbb{Q}_{\ell}(j)\right)\right) .
\end{aligned}
$$


For further discussion of the map (4.23) in this case, cf. [Ja3, §12].

(ii) $k=a$ number field or a global function field $(r=1)$. Here one expects $D^{b}\left(\mathscr{M} \mathscr{M}_{k}\right) \stackrel{\sim}{\rightarrow} \operatorname{grad} \mathscr{M} \mathscr{M}_{k}$, where $\operatorname{grad} \mathscr{M} \mathscr{M}_{k}$ is the category of $\mathbb{Z}$ graded objects $\bigoplus A^{j}$ in $\mathscr{M}_{\mathscr{M}_{k}}$ for which $A^{j}=0$ for almost all $j \in \mathbb{Z}$. For a smooth projective variety $X / k$ one expects a short exact sequence

$$
0 \rightarrow \operatorname{Ext}_{\mathscr{M K}_{k}}^{1}\left(1, h^{i-1}(X)(j)\right) \rightarrow H_{\mathscr{M}^{i}}^{i}(X, \mathbb{Q}(j)) \rightarrow \operatorname{Hom}_{\mathscr{M}_{k}}\left(1, h^{i}(X)(j)\right) \rightarrow 0
$$

This is related to the conjecture of Beilinson and Bloch that the Abel-Jacobi maps are injective on $\mathrm{CH}^{*}(X)_{\text {hom }} \otimes \mathbb{Q}$. For further discussion of the expected properties of the realization maps (4.17) in this case we refer the readers to [Nek; BK, FPR, Ja3]. Here we only point out the following two consequences. If $H_{\mathscr{M}}^{i}(X, \mathbb{Q}(j))_{0}$ denotes the kernel of the realization maps into $H^{i}\left(\bar{X}, \mathbb{Q}_{\ell}(j)\right) \quad(\ell \neq \operatorname{char} k)$ or $H^{i}\left(X \times_{\sigma} \mathbb{C}, \mathbb{Q}(j)\right) \quad(\operatorname{char} k=0, \sigma: k \hookrightarrow \mathbb{C}$ some embedding), then

$$
J=H_{\mathscr{M}}^{\cdot}(X, \mathbb{Q}(\cdot))_{0}
$$

should be an ideal of square zero (cf. [Bei2, 8.5.1; Bei3, 5.7]). Also the hard Lefschetz for $h^{\cdot}(X)$ should imply a hard Lefschetz for $H_{\mathscr{M}}^{\cdot}(X, \mathbb{Q}(\cdot))_{0}$, i.e., if $L \in C H^{1}(X)$ is the class of a hyperplane section, then the map

$$
L^{d-i+1}: H_{\mathscr{K}}^{i}(X, \mathbb{Q}(j))_{0} \rightarrow H_{\mathscr{M}}^{2 d-i+2}(X, \mathbb{Q}(j+d-i+1))_{0}
$$

should be an isomorphism for $i-1 \leq d$ (cf. [Bei3, Conjecture 5.3]).

(d) One may formulate still another version of Beilinson's conjecture (more general than version 3 , but still absolute and not "relative" as version 4), by postulating the existence of a suitable category $\mathscr{M} \mathscr{M}_{k}$ of mixed motives over $k$, and of complexes

$$
\underline{R \Gamma}_{Z}(X, j), \underline{R \Gamma^{\prime}}(X, b) \quad(j, b \in \mathbb{Z})
$$

in $D^{b}\left(\mathscr{M} \mathscr{M}_{k}\right)$ for arbitrary algebraic $k$-schemes $X$ and closed subschemes $Z \subset X$ forming something like a "derived" twisted Poincaré duality theory (cf. $[\mathbf{J 3}, 11.3]$ ). One would deduce this from version 4 by putting

$$
\begin{aligned}
& \underline{R \Gamma}_{Z}(X, j)=\left(a_{Z}\right)_{*} i \mathbb{Q}_{\mathscr{M}, X}(j), \\
& \underline{R \Gamma^{\prime}}(X, b)=\left(a_{X}\right)_{*} a_{X}^{!} \mathbb{Q}_{\mathscr{M}, \operatorname{Spec} k}(-b),
\end{aligned}
$$

where $a_{X}: X \rightarrow$ Spec $k$ is the structural morphism and $i: Z \hookrightarrow X$ is the closed immersion. The functors

$$
\begin{aligned}
&(Z \subset X) \mapsto H_{M, Z}^{i}(X, j)=H^{i}\left(\underline{R \Gamma_{Z}}(X, j)\right), \\
& X \mapsto H_{a}^{M}(X, b)=H^{-a}(\underline{R \Gamma}(X, b))
\end{aligned}
$$

would from a twisted Poincaré duality theory with values in $\mathscr{M}_{\mathscr{M}}$ is indicated in 2.5.

Depending on the point of view, the following lemma could be regarded as an "application" or as a test for the philosophy of motivic sheaves (this property of motivic cohomology was independently conjectured by Beilinson and Soulé [Be1, 2.2.2; Sou2, 2.9]. 
LEMMA 4.13. It is a consequence of the formalism of (mixed) motivic sheaves that

$$
H_{\mathscr{M}}^{i}(X, \mathbb{Q}(j))=0 \text { for } i<0,
$$

if $X$ is smooth over a field $k$.

Proof. If $a: X \rightarrow \operatorname{Spec} k$ is the structural morphism, we would have

$$
H_{\mathscr{M}}^{i}(X, \mathbb{Q}(j))=\operatorname{Hom}_{D^{b}\left(\mathscr{M} \mathscr{K}_{k}\right)}\left(1, a_{*} \mathbb{Q}_{\mathscr{M}}(j)[i]\right)
$$

as in (4.22). Now $a_{*} \mathbb{Q}_{\mathscr{M}}$ is a complex that is concentrated in degrees $\geq 0$; this follows, for example, from the property that

$$
r_{\ell} H^{\nu}\left(a_{*} \mathbb{Q}_{\mathscr{M}}(j)\right)=H^{\nu}\left(r_{\ell} a_{*} \mathbb{Q}_{\mathscr{M}}(j)\right)=H^{\nu}\left(\bar{X}, \mathbb{Q}_{\ell}(j)\right)=0 \text { for } \nu>0,
$$

and the faithfulness of $r_{\ell}$ on $\mathscr{M} \mathscr{M}_{k}$. Hence the above group vanishes for $i<0 \quad$ ( 1 is placed in degree zero), by standard properties of the derived category.

If $X$ is a smooth variety, then $K_{m}(X)=0$ for $m<0$, and hence trivially $H_{\mathscr{M}}^{i}(X, \mathbb{Q}(j))=K_{2 j-i}(X)^{(j)}=0$ for $i>2 j$. It is reassuring to know that this is also a consequence of the motivic picture.

LEMMA 4.14. The formalism of motivic sheaves implies that

$$
\operatorname{Hom}_{D^{b}\left(\mathscr{M}_{k}\right)}\left(1, a_{*} \mathbb{Q}_{\mathscr{M}}(j)[i]\right)=0 \text { for } i>2 j,
$$

if $a: X \rightarrow \operatorname{Spec} k$ is smooth.

The proof uses the following general fact.

LEMMA 4.15. Let $\mathscr{C}$ be an abelian category with a weight filtration such that the pure objects are simisimple. Then

$$
\operatorname{Ext}_{\mathscr{C}}^{\nu}(N, M)=0
$$

for objects $M, N$ in $\mathscr{C}$, provided

$$
\min \{\text { weights of } M\}>\max \{\text { weights of } N\}-\nu .
$$

Proof. By induction on $\nu$. For every $\nu$, an obvious devissage via the weight filtrations of $M$ and $N$ reduces the question to the case that $M$ and $N$ are pure of weights $m$ and $n$, respectively. Then $\operatorname{Hom}_{\mathscr{M}}(N, M)=0$ if $m \neq n$. For $\nu=1$ consider an extension

$$
0 \rightarrow M \rightarrow E \rightarrow N \rightarrow 0
$$

and the subextension

$$
0 \rightarrow W_{n} M \rightarrow W_{n} E \rightarrow N \rightarrow 0 .
$$

Since $m>n-1$, we have either $m>n$ or $m=n$. In the first case $W_{n} M=0$, and $W_{n} E$ provides a splitting of the given extension. In the second case $E$ is pure of weight $n$, and the sequence splits by the assumed semisimplicity. For $\nu>1$, every $\chi \in \operatorname{Ext}_{\mathscr{C}}^{\nu}(N, M)$ is the Yoneda product 
of $\chi_{1} \in \operatorname{Ext}_{\mathscr{C}}^{\nu-1}(N, X)$ and $\chi_{2} \in \operatorname{Ext}_{\mathscr{C}}^{1}(X, M)$ for some object $X$ in $\mathscr{C}$. If $\chi_{1}$ and $\chi_{2}$ are nonzero, then

$$
m+1 \leq \text { weights of } X \leq n-\nu+1
$$

by induction, and hence the claim for $\nu$.

Proof OF 4.14. By the Ext spectral sequence, it suffices to show that

$$
\operatorname{Ext}_{\mathscr{M K}_{k}}^{p}\left(1, H^{q}\left(a_{*} \mathbb{Q}_{\mathscr{M}}(j)\right)\right)=0 \text { for } p+q>2 j .
$$

However, the weights of $H^{q}\left(a_{*} \mathbb{Q}_{\mathscr{M}}(j)\right)$ are $\geq q-2 j$, since this holds for $h^{q}\left(\bar{X}, \mathbb{Q}_{\ell}(j)\right)=r_{\ell} H^{q}\left(a_{*} \mathbb{Q}_{\mathscr{M}}(j)\right)$ by results of Deligne [De3, 3.3.5]. (In the case of smooth projective $X$ we even know that $H^{q}\left(a_{*} \mathscr{Q}_{\mathscr{M}}(j)\right)=h^{q}(X)(j)$ is pure of weight $q-2 j$.) Hence the vanishing follows from Lemma 4.15.

\section{Murre's filtration}

The following concrete proposal for a filtration on Chow groups was made by Murre [Mur2, 1.4], based on his investigations on decompositions of Chow motives (cf. [Mur1]). Let $X$ be an irreducible smooth projective variety over a field $k$, and assume that the Künneth components $\pi_{j}^{\text {hom }}$ of the diagonal are algebraic (for some fixed Weil cohomology theory), for $j=0, \ldots, 2 d$, where $d=\operatorname{dim}(X)$. Then $\left\{\pi_{j}^{\text {hom }}\right\}$ forms an orthogonal set of idempotents in

$$
C H^{d}(X \times X)_{\mathbb{Q}} / \sim_{\text {hom }},
$$

with $\sum_{j=0}^{2 d} \pi_{j}^{\text {hom }}=$ class of the diagonal $\Delta=\mathrm{id}$ as a correspondence.

CONJECTURE 5.1 (Murre). (A) The $\pi_{j}^{\text {hom }}$ lift to an orthogonal set of idempotents $\left\{\pi_{j}\right\}$ in $C H^{d}(X \times X)_{\mathbb{Q}}$, with $\sum_{j=0}^{2 d} \pi_{j}=\Delta=\mathrm{id}$.

(B) The correspondences $\pi_{2 j+1}, \ldots, \pi_{2 d}$ act as zero on $C H^{j}(X)_{\mathbb{Q}}$.

(C) Let $F^{\nu} C H^{j}(X)_{\mathbb{Q}}=\operatorname{Ker} \pi_{2 j} \cap \operatorname{Ker} \pi_{2 j-1} \cap \cdots \cap \operatorname{Ker} \pi_{2 j-\nu+1}$. Then $F$ is independent of the choice of the $\pi_{j}$.

(D) $F^{1} C H^{j}(X)_{\mathbb{Q}}=C H^{j}(X)_{\text {hom, } \mathbb{Q}}$.

In fact, Murre stated the following stronger form:

Strong Conjecture 5.1. This is the same as Conjecture 5.1, except that (B) is replaced by

(strong B) The correspondences $\pi_{0}, \ldots, \pi_{j-1}$ and $\pi_{2 j+1}, \ldots, \pi_{2 d}$ act as zero on $\mathrm{CH}^{j}(X)_{\mathbb{Q}}$.

The main result of this section is

THEOREM 5.2. Murre's conjecture is equivalent to version 1 of Beilinson's conjecture, and the filtrations coincide; i.e., if one filtration exists then the other does and they agree. The same is true for the strong forms. 
Before we start with the proof, we explain the motivic background. If we start from version 3 of Beilinson's conjecture, we have a ring isomorphism

$$
C H^{d}(X \times X)_{\mathbb{Q}} \stackrel{\sim}{\rightarrow} \operatorname{End}_{D^{b}\left(M_{k}\right)}(R(X))
$$

(cf. (4.2)) and a decomposition in $D^{b}\left(\mathscr{M}_{k}\right)$

$$
R(X)=\bigoplus_{i \geq 0} h^{i}(X)[-i]
$$

(cf. Lemma 4.3). Any such decomposition gives an orthogonal set of idempotents $\left\{\pi_{j}\right\}$ as in $(A)$, by letting $\pi_{j}: R(X) \rightarrow h^{j}(X)[-j] \rightarrow R(X)$ be the projection on the $j$ th factor. Note that the image of $\pi_{j}$ in

$$
C H^{d}(X \times X)_{\mathbb{Q}} / \sim_{\mathrm{hom}} \stackrel{\sim}{\rightarrow} \bigoplus_{i \geq 0} \operatorname{End}_{\mathscr{M}_{k}}\left(h^{i}(X)\right)
$$

is in fact $\pi_{j}^{\text {hom }}$. The explicit description of Beilinson's filtration in Remark $4.5(\mathrm{a})$, namely,

$$
F^{\nu} C H^{j}(X)_{\mathbb{Q}}=\bigoplus_{i=0}^{2 j-\nu} \operatorname{Hom}_{D^{b}\left(\mathscr{M M}_{k}\right)}\left(1, h^{i}(X)(j)[2 j-i]\right),
$$

immediately implies the properties (B)-(D). As a motivation for the following considerations, we recall that under the full embedding $\mathscr{C O M M}_{k} \hookrightarrow D^{b}\left(\mathscr{M M}_{k}\right)$ we have a correspondence

$$
\text { Chow motive } M_{i}=\left(X, \pi_{i}, 0\right)_{\text {rat }} \leftrightarrow h^{i}(X)[-i] \in O b\left(D^{b}\left(\mathscr{M M}_{k}\right)\right)
$$

and that

$$
\text { (5.1) } \operatorname{Hom}_{D^{b}\left(\mathscr{M} \mathscr{K}_{k}\right)}\left(h^{i}(X)[-i], h^{i^{\prime}}(X)\left[-i^{\prime}\right]\right)= \begin{cases}0 & \text { for } i<i^{\prime}, \\ \operatorname{End}_{\mathscr{M}_{k}}\left(h^{i}(X)\right) & \text { for } i=i^{\prime}\end{cases}
$$

We now give a proof of Theorem 5.2 that does not mention mixed motives. Let $X$ be as above.

Proposition 5.3. Assume

$$
C H^{d}(X \times X)_{\text {hom }, \mathbb{Q}} \text { is a nilpotent ideal of } C H^{d}(X \times X)_{\mathbb{Q}} .
$$

Then Conjecture 5.1(A) holds.

This follows from

LEMMA 5.4. Let $A$ be a ring with unit (not necessarily commutative), and let $I \subset A$ be a nilpotent two-sided ideal. Then every set $\left\{e_{1}, \ldots, e_{m}\right\}$ of pairwise orthogonal idempotents (i.e., $e_{i} e_{j}=\delta_{i, j} e_{i}$ ) in $A / I$ can be lifted to a set $\left\{\pi_{1}, \ldots, \pi_{m}\right\}$ of pairwise orthogonal idempotents in $A$. If $\left\{\pi_{1}^{\prime}, \ldots, \pi_{m}^{\prime}\right\}$ is another such lifting, then there is an element $\eta \in I$ such that $\pi_{i}^{\prime}=$ 
$(1-\eta) \pi_{i}(1-\eta)^{-1}$ for $i=1, \ldots, m$. If $\sum_{i=1}^{m} e_{i}=1$, then necessarily $\sum_{i=1}^{m} \pi_{i}=1$.

PROof. By induction on the index of nilpotency we may assume $I^{2}=0$. The proof proceeds by induction on $m$. The case $m=1$ is probably well known; we found it as Beilinson's lemma in [Mur1, 7.3]. For $m>1$ assume that we have already an orthogonal lifting $\left\{\pi_{1}, \ldots, \pi_{m}\right\}$ of $\left\{e_{1}, \ldots, e_{m}\right\}$ and a lifting $\pi_{m+1}^{\prime}=\left(\pi_{m+1}^{\prime}\right)^{2}$ of $e_{m+1}$ which is orthogonal to $\pi_{1}, \ldots, \pi_{n}$ for some $0 \leq n<m$ (new induction on $n$ starting with the empty case $n=0)$. Let

$$
\pi_{n+1} \pi_{m+1}^{\prime}=\varepsilon \in I .
$$

Then $\varepsilon=\pi_{n+1} \varepsilon \pi_{m+1}^{\prime}$ and

$$
\begin{aligned}
\pi_{n+1}(1-\varepsilon) \pi_{m+1}^{\prime}(1+\varepsilon) & =\pi_{n+1}\left(\pi_{m+1}^{\prime}+\pi_{m+1}^{\prime} \varepsilon-\varepsilon \pi_{m+1}^{\prime}\right) \\
& =\varepsilon+\varepsilon^{2}-\pi_{n+1} \varepsilon \pi_{m+1}^{\prime}=0 .
\end{aligned}
$$

Moreover, $\pi_{i} \varepsilon=\pi_{i} \pi_{n+1} \pi_{m+1}^{\prime}=0=\pi_{n+1} \pi_{m+1}^{\prime} \pi_{i}=\varepsilon \pi_{i}$ for $i \leq n$, so that

$$
\pi_{m+1}^{\prime \prime}=(1-\varepsilon) \pi_{m+1}^{\prime}(1+\varepsilon)
$$

is an idempotent lifting $e_{m+1}$, which is orthogonal to $\left\{\pi_{1}, \ldots, \pi_{n}\right\}$ and in addition satisfies $\pi_{n+1} \pi_{m+1}^{\prime \prime}=0$. Now let

$$
\pi_{m+1}^{\prime \prime} \pi_{n+1}=\varepsilon^{\prime} \in I
$$

Then $\varepsilon^{\prime}=\pi_{m+1}^{\prime \prime} \varepsilon^{\prime} \pi_{n+1}$, and

$$
\begin{aligned}
\pi_{i} \varepsilon^{\prime} & =0=\varepsilon^{\prime} \pi_{i} \text { for } i \leq n, \\
\pi_{n+1} \pi_{m+1}^{\prime \prime} & =0, \\
\pi_{n+1} \varepsilon^{\prime} & =0 .
\end{aligned}
$$

As above we deduce that $\pi_{m+1}=\left(1+\varepsilon^{\prime}\right) \pi_{m+1}^{\prime \prime}\left(1-\varepsilon^{\prime}\right)$ is an idempotent lifting $e_{m+1}$, which is now orthogonal to $\left\{\pi_{1}, \ldots, \pi_{n+1}\right\}$, since

$$
\begin{aligned}
\pi_{m+1} \pi_{n+1} & =\left(\pi_{m+1}^{\prime \prime}+\varepsilon^{\prime} \pi_{m+1}^{\prime \prime}-\pi_{m+1}^{\prime \prime} \varepsilon^{\prime}\right) \pi_{n+1} \\
& =\varepsilon^{\prime}+\left(\varepsilon^{\prime}\right)^{2}-\pi_{m+1}^{\prime \prime} \varepsilon^{\prime} \pi_{n+1}=0 .
\end{aligned}
$$

By induction on $n$ we get an orthogonal set of idempotents $\left\{\pi_{1}, \ldots, \pi_{m+1}\right\}$ lifting $\left\{e_{1}, \ldots, e_{m+1}\right\}$.

For the uniqueness assume that we have already conjugated a second orthogonal lifting $\left\{\pi_{1}^{\prime}, \ldots, \pi_{m+1}^{\prime}\right\}$ such that $\pi_{i}^{\prime}=\pi_{i}$ for $i \leq m$. By the Beilinson lemma we have

$$
\pi_{m+1}^{\prime}=(1-\varepsilon) \pi_{m+1}(1+\varepsilon)=\pi_{m+1}-\varepsilon \pi_{m+1}+\pi_{m+1} \varepsilon
$$

for some $\varepsilon \in I$. Since by assumption $\pi_{i} \pi_{m+1}^{\prime}=0=\pi_{m+1}^{\prime} \pi_{i}$ for $i \leq m$, we must have

$$
\pi_{i} \varepsilon \pi_{m+1}=0=\pi_{m+1} \varepsilon \pi_{i} \text { for } i \leq m .
$$


Putting $\eta=\varepsilon \pi_{m+1}+\pi_{m+1} \varepsilon \in I$, one easily computes

$$
\begin{aligned}
\pi_{i} & =(1-\eta) \pi_{i}(1+\eta) \text { for } i \leq m, \\
\pi_{m+1}^{\prime} & =(1-\eta) \pi_{m+1}(1+\eta),
\end{aligned}
$$

which proves the claim.

Finally, if $\sum_{i=1}^{m} \pi_{i}=1+\varepsilon$ for $\varepsilon \in I$, then $1+\varepsilon=(1+\varepsilon)^{2}=1+2 \varepsilon$, and hence $\varepsilon=0$.

Proposition 5.5. Assume one has a descending filtration $F^{\cdot}$ on $C H^{j}(X)_{\mathbb{Q}}$ with the following properties:

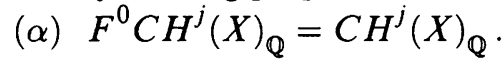

( $\beta$ ) The action of $C H^{d}(X \times X)_{\mathbb{Q}}$ on $C H^{j}(X)_{\mathbb{Q}}$ respects $F^{\cdot}$, factors through homological equivalence on $\mathrm{Gr}_{F}^{\cdot} C H^{j}(X)_{\mathbb{Q}}$, and one has

$$
\pi_{i}^{\text {hom }}=\delta_{i, 2 j-\nu} \cdot \text { id on } \operatorname{Gr}_{F}^{\nu} C H^{j}(X)_{\mathbb{Q}} .
$$

(y) $F^{N} C H^{j}(X)_{\mathbb{Q}}=0$ for some $N \in \mathbb{N}$.

Then for any liftings $\pi_{0}, \ldots, \pi_{2 d} \in C H^{d}(X \times X)_{\mathbb{Q}}$ of $\pi_{0}^{\text {hom }}, \ldots, \pi_{2 d}^{\text {hom }}$ (not necessarily idempotent or orthogonal) and for every $\mu \geq N$, the following holds.

$\left(\mathrm{B}^{\prime}\right)$ The correspondences $\pi_{2 j+1}^{\mu}, \ldots, \pi_{2 d}^{\mu}$ and $\pi_{2 j-N}^{\mu}, \pi_{2 j-N-1}^{\mu}, \ldots, \pi_{0}^{\mu}$ act as zero on $\mathrm{CH}^{j}(X)_{\mathbb{Q}}$.

$$
\begin{aligned}
F^{\nu} C H^{j}(X)_{\mathbb{Q}} & =\operatorname{Ker} \pi_{2 j}^{\mu} \cap \operatorname{Ker} \pi_{2 j-1}^{\mu} \cap \cdots \cap \operatorname{Ker} \pi_{2 j-\nu+1}^{\mu} \\
& =\operatorname{Im} \pi_{2 j-\nu}^{\mu}+\operatorname{Im} \pi_{2 j-\nu-1}^{\mu}+\cdots+\operatorname{Im} \pi_{2 j-\mu+1}^{\mu}
\end{aligned}
$$

for $\nu \geq 0$.

Proof. By assumptions $(\beta)$ and $(\gamma), \pi_{2 d}, \ldots, \pi_{2 j+1}$ and $\pi_{2 j-N}, \ldots, \pi_{0}$ act as zero on $\mathrm{Gr}_{F}^{\cdot} C H^{j}(X)_{\mathbb{Q}}$. This obviously implies $\left(\mathbf{B}^{\prime}\right)$, since the filtration has length $N$ by $(\gamma)$. Exactly the same argument shows that $\pi_{2 d}^{N-\nu}, \pi_{2 d-1}^{N-\nu}$, $\ldots, \pi_{2 j-\nu+1}^{N-\nu}$ act as zero on $F^{\nu} C H^{j}(X)_{\mathbb{Q}}$ for $\nu \geq 0$. Hence

$$
F^{\nu} C H^{j}(X)_{\mathbb{Q}} \subseteq \operatorname{Ker} \pi_{2 j}^{\mu} \cap \operatorname{Ker} \pi_{2 j-1}^{\mu} \cap \cdots \cap \operatorname{Ker}_{2 j-\nu+1}^{\mu} .
$$

The converse inclusion follows by induction on $\nu$. Starting with the trivial case $\nu=0$, we have to show

$$
F^{\nu \cdot} \cap \operatorname{Ker} \pi_{2 j-\nu}^{\mu} \subseteq F^{\nu+1}
$$

But since $\pi_{2 j-\nu}^{\mu}$ is the identity on $\operatorname{Gr}_{F}^{\nu} C H^{j}(X)_{\mathbb{Q}}$, we have $x-\pi_{2 j-\nu}^{\mu}(x) \in$ $F^{\nu+1}$ for every $x \in F^{\nu}$. The proof of the second equality in $\left(C^{\prime}\right)$ is dual: Since $\pi_{2 j-\nu}^{\nu}, \pi_{2 j-\nu-1}^{\nu}, \ldots, \pi_{0}^{\nu}$ act as zero on $C H^{j}(X)_{\mathbb{Q}} / F^{\nu}$, we have the inclusion

$$
F^{\nu} C H^{j}(X)_{\mathbb{Q}} \supseteq \operatorname{Im} \pi_{2 j-\nu}^{\mu}+\operatorname{Im} \pi_{2 j-\nu-1}^{\mu}+\cdots+\operatorname{Im} \pi_{2 j-N+1}^{\mu} .
$$


The equality follows by descending induction on $\nu$, starting with the trivial case $\nu=N$. The induction step

$$
F^{\nu} C H^{j}(X)_{\mathbb{Q}} \subseteq F^{\nu+1} C H^{j}(X)_{\mathbb{Q}}+\operatorname{Im} \pi_{2 j-\nu}^{\mu}
$$

is clear from the fact that $\pi_{2 j-\nu}$ is the identity on $\operatorname{Gr}_{F}^{\nu} C H^{j}(X)_{\mathbb{Q}}$.

REMARKs 5.6. (a) If the $\pi_{i}$ are idempotents, then $\pi_{i}^{\mu}=\pi_{i}$ for all $i$. If the $\pi_{i}$ are pairwise orthogonal, then

$$
F^{\nu} C H^{j}(X)_{\mathbb{Q}}=\operatorname{Im}\left(\pi_{2 j-\nu}^{\mu}+\pi_{2 j-\nu-1}^{\mu}+\cdots+\pi_{2 j-N+1}^{\mu}\right)
$$

for all $\mu \geq N$. In fact, by Proposition 5.5

$$
F^{\nu} C H^{j}(X)_{\mathbb{Q}}=\operatorname{Im} \pi_{2 j-\nu}^{\mu+1}+\operatorname{Im} \pi_{2 j-\nu-1}^{\mu+1}+\cdots+\operatorname{Im} \pi_{2 j-N+1}^{\mu+1},
$$

and by orthogonality this is contained in the right-hand side of (5.2), by the relation $\sum \pi_{i}^{\mu+1}\left(x_{i}\right)=\left(\sum \pi_{i}^{\mu}\right)\left(\sum \pi_{r}\left(x_{r}\right)\right)$. The converse inclusion in (5.2) follows trivially from Proposition 5.5.

(b) A filtration as in Beilinson's conjecture (any version) satisfies $(\alpha)$ and $(\gamma)$ (by definition) and $(\beta)$ (as was observed after the formulation of version 1). The above assumptions are much weaker, since one only needs a filtration on $C H^{j}(X)_{\mathbb{Q}}$ and properties of self-correspondences acting on it. In fact, even if one deduces $(\alpha),(\beta)$, and $(\gamma)$ from Beilinson's conjecture (version 1 ), one only needs to consider $X, X \times X, X^{3}$, and $X^{4}$.

Corollary 5.7. A filtration $F^{\cdot}$ on $C H^{j}(X)_{\mathbb{Q}}$ satisfying Proposition $5.5(\alpha),(\beta)$, and $(\gamma)$ is unique. In particular, Beilinson's conjectured filtration is unique.

We can now prove that Beilinson's conjecture implies Murre's conjecture: Indeed, let $F^{*}$ be Beilinson's filtration as in Conjecture 2.1. Then it follows from its properties (b) and (c) that

$$
F^{r} C H^{d}(X \times X)_{\mathbb{Q}} \circ F^{s} C H^{d}(X \times X)_{\mathbb{Q}} \subseteq F^{r+s} C H^{d}(X \times X)_{\mathbb{Q}}
$$

under composition of correspondences. Hence

$$
F^{1} C H^{d}(X \times X)_{\mathbb{Q}} \stackrel{(\mathrm{a})}{=} C H^{d}(X \times X)_{\text {hom, } \mathbb{Q}}
$$

is a nilpotent ideal by (e), and we get part (A) of Murre's conjecture by Proposition 5.3. By Proposition 5.5 and Remark 5.6(a), every set $\left\{\pi_{i}\right\}$ of idempotents (not necessarily orthogonal) lifting $\left\{\pi_{i}^{\text {hom }}\right\}$ satisfies part (B), if $F^{\cdot}$ satisfies (e), and (strong B), if $F^{\cdot}$ satifies (strong e). Moreover, Murre's filtration defined via these $\pi_{i}$ agrees with Beilinson's, and in particular, we obtain (C), with the additional information that the $\pi_{i}$ do not have to be orthogonal. Finally, Murre's (D) follows from property (a) of Beilinson's filtration $F^{\cdot}$.

For the converse implication in Theorem 5.2 the following fact (cf. (5.1)) is crucial. 
Proposition 5.8. Let $\left\{\pi_{i}\right\}=\left\{\pi_{i}^{X}\right\}$ be an orthogonal set of idempotents lifting $\left\{\pi_{i}^{\mathrm{hom}}\right\}=\left\{\pi_{i}^{X, \mathrm{hom}}\right\}$. Let $M_{i}=\left(X, \pi_{i}, 0\right)_{\mathrm{rat}}$ be the Chow motive associated to $\pi_{i}(i=0, \ldots, 2 d)$. If Murre's conjecture holds for $X \times X$, then

$$
\operatorname{Hom}_{\text {ESeM }_{k}}\left(M_{i}, M_{j}\right)= \begin{cases}0 & \text { for } i<j, \\ \operatorname{End}_{\mathscr{M}_{k}}\left(h^{i}(X)\right) & \text { for } i=j .\end{cases}
$$

More generally, let $Y$ be a smooth projective variety of pure dimension e over $k$, let $\left\{\pi_{0}^{Y}, \ldots, \pi_{2 e}^{Y}\right\} \subseteq C H^{2 e}(Y \times Y)_{\mathbb{Q}}$ be an orthogonal set of idempotents lifting the set $\left\{\pi_{0}^{Y, \text { hom }}, \ldots, \pi_{2 e}^{Y, \text { hom }}\right\}$ of Künneth components of the diagonal $\Delta_{Y}$ for $Y$, and denote by $N_{j}=\left(Y, \pi_{j}, 0\right)_{\text {rat }}$ the Chow motive associated to $\pi_{j}^{Y}(j=0, \ldots, 2 e)$. If Murre's conjecture holds for $X \times Y$, then

$$
\operatorname{Hom}_{\text {gran }_{k}}\left(M_{i}, N_{j}\right)= \begin{cases}0 & \text { for } i<j, \\ \operatorname{Hom}_{\mathscr{N}_{k}}\left(h^{i}(X), h^{i}(Y)\right) & \text { for } i=j .\end{cases}
$$

Proof. Obviously it suffices to show the second claim. Denote by $\alpha \mapsto$ ${ }^{t} \alpha$ the transposition on $C H^{d}(X \times X)_{\mathbb{Q}}$, i.e., the anti-involution induced by interchanging the factors of $X \times X$. Since clearly

$$
{ }^{t} \pi_{i}^{\text {hom }}=\pi_{2 d-i}^{\text {hom }},
$$

$\left\{\tilde{\pi}_{0}, \ldots, \tilde{\pi}_{2 d}\right\}$, with $\tilde{\pi}_{i}={ }^{t} \pi_{2 d-i}$, is an orthogonal set of idempotents lifting $\left\{\pi_{0}^{\text {hom }}, \ldots, \pi_{2 d}^{\text {hom }}\right\}$. For $\alpha \in C H^{d}(X \times X)_{\mathbb{Q}}$ and $\beta \in C H^{e}(Y \times Y)_{\mathbb{Q}}$ denote by $\alpha \times \beta \in C H^{d+e}(X \times X \times Y \times Y)_{\mathbb{Q}}$ the external product, regarded as a correspondence on $X \times Y$ via the isomorphism

$$
C H^{d+e}(X \times X \times Y \times Y)_{\mathbb{Q}}^{\stackrel{\varphi^{*}}{\sim}} C H^{d+e}(X \times Y \times X \times Y)
$$

induced by $\varphi: X \times Y \times X \times Y \stackrel{\sim}{\rightarrow} X \times X \times Y \times Y,\left(x_{1}, y_{1}, x_{2}, y_{2}\right) \mapsto$ $\left(x_{1}, x_{2}, y_{1}, y_{2}\right)$. Then the external products

$\tilde{\pi}_{i}^{X} \times \pi_{j}^{Y} \in C H^{d+e}((X \times Y) \times(X \times Y)), \quad i=0, \ldots, 2 d, j=0, \ldots, 2 e$,

form an orthogonal set of idempotents, by the formula

$$
(\alpha \times \beta) \circ\left(\alpha^{\prime} \times \beta^{\prime}\right)=\alpha \circ \alpha^{\prime} \times \beta \circ \beta^{\prime}
$$

for $\alpha, \alpha^{\prime} \in C H^{d}(X \times X)_{\mathbb{Q}}$ and $\beta, \beta^{\prime} \in C H^{e}(Y \times Y)_{\mathbb{Q}}$, which is easily established (cf. [Ma, p. 448, lemma]; use the formulae $\left(f_{1} \times f_{2}\right)^{*}(\alpha \times \beta)=$ $f_{1}^{*} \alpha \times f_{2}^{*} \beta,\left(f_{1} \times f_{2}\right)_{*}(\alpha \times \beta)=\left(f_{1}\right)_{*} \alpha \times\left(f_{2}\right)_{*} \beta$, and $(\alpha \times \beta) \cdot\left(\alpha^{\prime} \times \beta^{\prime}\right)=$ $\left.\alpha \cdot \alpha^{\prime} \times \beta \cdot \beta^{\prime}\right)$. Furthermore, the Künneth formula shows that

$$
\Pi_{r}=\sum_{i+j=r} \tilde{\pi}_{i}^{X} \times \pi_{j}^{Y}
$$

is a lifting of the $r$ th Künneth component of the diagonal $\Delta_{X \times Y}$ for $X \times Y$. 
Part (B) of Murre's conjecture for $X \times Y$ asserts that $\Pi_{2 d+1}, \Pi_{2 d+2}, \ldots$, $\Pi_{2 d+2 e}$, regarded as correspondences on $X \times Y$, act as zero on $C H^{d}(X \times Y)_{\mathbb{Q}}$. Together with the mentioned orthogonality this implies that $\tilde{\pi}_{i}^{X} \times \pi_{j}^{Y}$ acts as zero on $C H^{d}(X \times Y)_{\mathbb{Q}}$ for all pairs $(i, j)$ with $i+j>2 d$. Hence

$$
\begin{aligned}
0 & =\left(\tilde{\pi}_{i}^{X} \times \pi_{j}^{Y}\right) C H^{d}(X \times Y)_{\mathbb{Q}} \quad \text { [application of } \tilde{\pi}_{i} \times \pi_{j} \text { ] } \\
& =\pi_{j}^{Y} C H^{d}(X \times Y)_{\mathbb{Q}}{ }^{t} \tilde{\pi}_{i}^{X} \quad \text { [composition with }{ }^{t} \tilde{\pi}_{i} \text { and } \pi_{j} \text { ] } \\
& =\pi_{j}^{Y} C H^{d}(X \times Y)_{\mathbb{Q}} \pi_{2 d-i}^{X} \quad \text { [by definition] } \\
& \left.=\operatorname{Hom}_{\operatorname{Cose}_{k}}\left(M_{2 d-i}, N_{j}\right) \quad \text { [by definition, cf. } \S 2\right],
\end{aligned}
$$

provided $i+j>2 d$, i.e., $2 d-i<j$. Here the second equality is an easy consequence of the definitions; cf. Lieberman's lemma in [K12, p. 73].

Part (D) of Murre's conjecture for $X \times Y$ implies that $C H^{d}(X \times Y)_{\text {hom, } \mathbb{Q}}=$ $F^{1} C H^{d}(X \times Y)_{\mathbb{Q}}=\operatorname{Ker} \Pi_{2 d}$. As above we obtain

$$
\begin{aligned}
\operatorname{Hom}_{\text {Ese }_{k}}\left(M_{i}, N_{i}\right) & =\left(\tilde{\pi}_{2 d-i}^{X} \times \pi_{i}^{Y}\right) C H^{d}(X \times Y) \\
& =\left(\tilde{\pi}_{2 d-i}^{X} \times \pi_{i}^{Y}\right) C H^{d}(X \times Y)_{\mathbb{Q}} / \sim_{\text {hom }} \\
& =\pi_{i}^{Y, \operatorname{hom}}\left(C H^{d}(X \times Y)_{\mathbb{Q}} / \sim_{\text {hom }}\right) \pi_{i}^{X, \text { hom }} \\
& =\operatorname{Hom}_{\mathscr{M}_{k}}\left(h^{i}(X), h^{i}(Y)\right) .
\end{aligned}
$$

Hence 5.8 is proved.

We now prove that Murre's conjecture implies Beilinson's conjecture: Let $X, Y$, and the notation be as in Proposition 5.8, and assume Murre's conjecture for $X, Y$, and $X \times Y$. Define the filtration $F^{\cdot}$ on $C H^{*}(X)_{Q}$ as in part (C) of Murre's conjecture from $\left\{\pi_{i}^{X}\right\}$, similarly for $Y$. Then (a) and (e) (resp. (strong e)) of Beilinson's conjecture follow, for this filtration, by definition, by (D) and by (B) and (strong B), respectively.

For the functoriality in part (c) of Beilinson's conjecture we use the following reinterpretation of $F^{\circ}$. Recall that one can define rational Chow groups of Chow motives; for a Chow motive $(X, p, 0)_{\text {rat }}$ we have

$$
C H^{j}\left((X, p, 0)_{\mathrm{rat}}\right)_{\mathbb{Q}}=p C H^{j}(X)_{\mathbb{Q}}=\operatorname{Im} p=\operatorname{Ker}(1-p)
$$

where $p: C H^{j}(X)_{\mathbb{Q}} \rightarrow C H^{j}(X)_{\mathbb{Q}}$ also denotes the endomorphism induced by the correspondence $p$. If $M$ is the Chow motive associated to $X$, then

$$
M=\bigoplus_{i=0}^{2 d} M_{i}
$$


by definition, and we have

$$
\begin{aligned}
F^{\nu} C H^{j}(X)_{\mathbb{Q}} & =\bigcap_{k=2 j-\nu+1}^{2 d} \operatorname{Ker} \pi_{k}^{X} \\
& =\bigoplus_{i=0}^{2 j-\nu} \pi_{i}^{X} C H^{j}(X)_{\mathbb{Q}} \\
& =\bigoplus_{i=0}^{2 j-\nu} C H^{j}\left(M_{i}\right)_{\mathbb{Q}}
\end{aligned}
$$

(this should be compared with the motivic considerations after Theorem 5.2). We have a similar description for $F^{*}$ on $C H^{j}(Y)_{\mathbb{Q}}$, and now Proposition 5.8 implies that every correspondence $\alpha \in C H^{d}(X \times Y)_{\mathbb{Q}}$ maps $F^{\nu} C H^{j}(X)_{\mathbb{Q}}$ to $F^{\nu} C H^{j}(Y)_{\mathbb{Q}}$. In fact, $\alpha$ maps $\bigoplus_{i=0}^{2 j-\nu} M_{i}$ to $\bigoplus_{i=0}^{2 j-\nu} N_{i}$ by Proposition 5.8 (in more down-to-earth terms, Proposition 5.8 says that $\pi_{k}^{Y} \alpha \pi_{i}^{X}=0$ for $i<k$, so that $\pi_{k}^{Y} \alpha F^{\nu} C H^{j}(X)_{\mathbb{Q}}=0$ for $\left.k>2 j-\nu\right)$.

In particular, $F^{*}$ is respected by $f_{*}$ for a morphism $f: X \rightarrow Y$, and the same follows for $f^{*}$ by interchanging the roles of $X$ and $Y$ (and considering Murre's conjecture for $\left.C H^{e}(Y \times X)_{\mathbb{Q}}\right)$.

For property (b) of Beilinson's conjecture we note that the intersection product coincides with the composition

$$
C H^{i}(X)_{\mathbb{Q}} \otimes C H^{j}(X)_{\mathbb{Q}} \rightarrow C H^{i+j}(X \times X)_{\mathbb{Q}} \stackrel{\Delta^{*}}{\rightarrow} C H^{i+j}(X)_{\mathbb{Q}}
$$

where the first arrow is the external product and the second one is the pullback for the diagonal $\Delta: X \rightarrow X \times X$. If we define a filtration $F^{\circ}$ on $C H^{i+j}(X \times X)_{\mathbb{Q}}$ by Murre's formula and the orthogonal idempotents

$$
\Pi_{r}=\sum_{i+j=r} \pi_{i} \times \pi_{j} \quad(r=0, \ldots, 4 d)
$$

lifting the Künneth components of the diagonal for $X \times X$, then the external product maps

$$
F^{\mu} C H^{i}(X)_{\mathbb{Q}} \otimes F^{\nu} C H^{j}(X)_{\mathbb{Q}}=\left(\bigoplus_{k=0}^{2 i-\mu} \pi_{k} C H^{i}(X)_{\mathbb{Q}}\right) \otimes\left(\bigoplus_{\ell=0}^{2 j-\nu} \pi_{\ell} C H^{j}(X)_{\mathbb{Q}}\right)
$$

to

$$
\bigoplus_{\substack{k, \ell \\ k+\ell \leq 2(i+j)-(\mu+\nu)}}\left(\pi_{k} \times \pi_{\ell}\right) C H^{i+j}(X \times X)_{\mathbb{Q}}=F^{\mu+\nu} C H^{i+j}(X \times X)_{\mathbb{Q}},
$$

and $\Delta^{*}$ maps $F^{\mu+\nu} C H^{i+j}(X \times X)_{\mathbb{Q}}$ to $F^{\mu+\nu} C H^{i+j}(X)_{\mathbb{Q}}$ by the previous step, if we assume Murre's conjecture for $X \times X \times X$.

Finally, property (d) of Beilinson's conjecture is clear from the formula

$$
\operatorname{Gr}_{F}^{\nu} C H^{j}(X)_{\mathbb{Q}} \cong C H^{j}\left(M_{2 j-\nu}\right)_{\mathbb{Q}}=\pi_{2 j-\nu} C H^{j}(X)_{\mathbb{Q}} .
$$


Thus, Theorem 5.2 is completely proved, in view of the uniqueness result proved in Corollary 5.7.

The status of Murre's conjecture is as follows [Mur2]: It is trivially true for curves. For surfaces and for threefolds of type $S \times C$, where $S$ is a surface and $C$ is a curve, Murre has proved (A), as well as (B) and (D) for a natural choice of idempotents. For surfaces he shows that his filtration is the natural one (for zero cycles the one considered by Bloch; cf. (1.9)), in support of (C). For abelian varieites, (A) follows from work of Shermenev, Deninger-Murre, and Künnemann (cf. [Kü1]) and for a natural choice of idempotents part of (B) follows from work of Beauville [Beau], and the rest of (B) is equivalent to a conjecture of Beauville.

REMARKs 5.9. (a) Murre's work and the considerations in $\S 3$ suggest finding $\pi_{i}$ supported on $Y_{i} \times X$, where $Y_{i}$ is of dimension $i$, for $0 \leq i \leq d$, and to take $\pi_{2 d-i}={ }^{t} \pi_{i}$. (Cf. also [Mur2, Question 3.3].)

(b) S. Saito defined another filtration on Chow groups without assuming any special conditions, and he showed that it agrees with Beilinson's filtration, given the formalism of mixed motives as in version 3 of Beilinson's conjecture. We refer the reader to [SaS] for this and for further interesting discussion.

(c) As we have seen, one may easily define filtrations on Chow groups that are exhaustive (as in Murre's definition) or that satisfy Proposition 5.5( $\beta$ ) (this is the case for the filtration $F_{\ell}^{\cdot}$ in the proof of Lemma 2.7 and for Saito's definition). It seems to be very hard to define filtrations that satisfy both $5.5(\beta)$ and $(\gamma)$.

\section{Appendix: A letter from Grothendieck to Illusie}

Buffalo le 3.5.1973

Cher Illusie,

Je t'envoie quelques afterthoughts de notre conversation mathématique sur les motifs. J'avais dit à tort que les isomotifs n'ont pas de "modules infinitésimaux", c'est-à-dire que si $i: S_{0} \rightarrow S$ est une immersion nilpotente, le foncteur image inverse de motifs est une équivalence de catégories. Cela doit être vrai en car. $p>0$ (plus généralement, si $\mathscr{O}_{S}$ est annulé par une puissance de $p$ ), pour la raison heuristique (qu'on peut expliciter entièrement lorsq'on travaille dans le contexte bien assis des schemas abéliens, ou des groupes de Barsotti-Tate) que lorsqu'on se ramène par dévissage au cas d'une nilimmersion d'ordre $1\left(J^{2}=0\right)$, on peut définir une obstruction à la déformation sur $S$ d'un homomorphisme (ou isomorphisme) de (pas iso) motif sur $S_{0}$, qui sera tuée par $p^{i}$ si $p^{i}$ tue $J$, donc qui sera tuée lorsqu'on passe aux isomotifs. Par contre, en caractéristique nulle, les schémas abéliens à isogénie près ont la même théorie des modules infinitésimaux que les schémas abéliens tout courts, et il faut s'attendre à la même chose pour les 
motifs et isomotifs. En termes des théories de systèmes de coefficients de de Rham ou de Hodge, l'élément de structure "filtration de DR" introduit bel et bien un élément de continuité, qui a pour effet de rendre faux le fait que pour ces coefficients, le foncteur image inverse par une nilimmersion soit une équivalence. Il semble donc qu'il faille bannir cette propriété (hors du cas des schémas de torsion) du yoga des "coefficients discrets". A moins qu'il se trouve que les besoins du formalisme (construction de foncteurs adjoints du type $R f_{*}$ etc.) nous impose de modifier la notion de faisceau de Hodge ou de DR sur un schéma $X$, en partant du genre de notion que nous avions regardée ensemble, et en passant ensuite aux catégories $\operatorname{Lim}$ des catégories correspondantes associées à $X^{\prime}$, où $X^{\prime}$ est réduit et $X^{\prime} \rightarrow \vec{X}$ est fini radiciel surjectif. Mais j'espère qu'il ne sera pas nécessaire de canuler ces notions ainsi. Une question liée est celle-ci: si $X$ est de car. 0 , un isomotif serein sur $X$ qui est "effectif de poids 1" définit-il bien un schéma abélien à isogénie près, ou seulement un schéma abélien à isogénie près au dessus d'un $X^{\prime}$ comme ci-dessus? Ce dernier devrait être le cas en tous cas en car. $p>0$, si on veut qu'un morphisme fini surjectif soit un morphisme de descente effective pour les isomotifs (et cela à son tour doit être vrai, étant vrai pour les $\mathbb{Q}_{\ell}$-faisceaux, si on veut que le foncteur isomotifs $\rightarrow \mathbb{Q}_{\ell}$-faisceaux commute aux opérations habituelles et est fidèle-et on le veut à tout prix!). Ainsi, en car. $p>0$, si $k$ est un corps, un isomotif effectif de poids 1 sur $k$ devrait être, non un schéma abélien à isogénie près sur $k$, mais sur la clôture parfaite de $k$ !

Je n'ai pas le coeur net non plus sur la nécessité de mettre du "iso" partout dans la théorie des motifs. Je ne serais pas tellement étonné qu'il y a en caractéristique nulle une théorie des motifs (pas iso), qui s'envoie dans les théories $\ell$-adiques (sur $\mathbb{Z}_{\ell}$, pas $\mathbb{Q}_{\ell}$ ) pour tout $\ell$. Pour ce qui est des coefficients de Hodge, il devrait être assez trivial de les définir "pas iso", de telle façon que les $\mathbb{Z}$-faisceaux de torsion algébriquement constructibles (sur $X$ de type fini sur $\mathbb{C}$ ) en forment une sous-catégorie pleine, et avec un foncteur vers les $\mathbb{Z}$-faisceaux algébriquement constructibles ("foncteur de Betti"). En caractéristique $p>0$, j'ai des doutes très sérieux pour l'existence d'une théorie des motifs pas iso du tout, à cause des phénomènes de $p$-torsion (surtout pour les schémas qui ne sont pas projectifs et lisses). Ainsi, si on admet la description de Deligne des "motifs mixtes" de niveau 1 comme le genre de chose permettant de définir un $H^{1}$ motivique d'un schéma pas projectif ou pas lisse, on voit que déjà pour une courbe algébrique sur un corps imparfait $k$, la construction ne peut fournir en général qu'un objet du type voulu sur la clôture parfaite de $k$. Par contre, il pourrait être vrai que seul la $p$-torsion canule, et qu'il suffise de localiser par tuage de $p$-torsion, c'est-à dire moralement de travailler avec des catégories $\mathbb{Z}[1 / p]$-linéaires. On aurait alors encore des foncteurs allant des "motifs" (pas iso) vers les $\mathbb{Z}_{\ell}$-faisceaux (quel que soit $\ell \neq p$ ) mais pas vers les $F$-cristaux, mais seulement vers les 
$F$-isocristaux. Dans cette théorie, on renoncerait donc simplement à regarder en car. $p$ des phénomènes de $p$-torsion. Pourtant il est "clair" que ceux-ci existent et sont fort intéressants, tout au moins pour les morphismes propres et lisses, et on a bien l'impression que la cohomologie cristalline (plus fine que DR) pas iso en donne la clef. (Au fait, Berthelot est-il parvenu à des conjectures plausibles à cet sujet?) On peut donc espérer que pour les motifs sereins et semi-simples fibre par fibre, on a des catégories sur $\mathbb{Z}$, pas seulement sur $\mathbb{Z}[1 / p]$, les Hom étant des $\mathbb{Z}$-modules de type finit. Cette impression peut être fondée par exemple sur le joli comportement des schémas abéliens sur le corps des fractions d'un anneau de val. discrète: dans la théorie de spécialisation, il se trouve qu'à un aucun moment la $p$-torsion ne canule.

Bien sûr, alors même qu'on arriverait à travailler avec des catégories des motifs pas iso, dans "l'état actuel de la science", pour en déduire une théorie de groupes de Galois motiviques, étant obligé de s'appuyer sur ce que Saavedra a rédigé, on est obligé à tensoriser tout par $\mathbb{Q}$, et on ne trouve que des groupes algébriques sur $\mathbb{Q}$ ou des extensions de $\mathbb{Q}$. Néanmoins, on a certainement dans l'idée que les "vrais" groupes de Galois motiviques (associés à des foncteurs-fibres comme la cohomologie $\ell$-adique, ou la cohomologie de Betti) sont des schémas en groupes sur $\mathbb{Z}_{\ell}$ et sur $\mathbb{Z}$ plutôt que sur $\mathbb{Q}_{\ell}$ et sur $\mathbb{Q}$, et par là on devrait rejoindre le point de vue des groupes de type arithmétique de gens comme Borel, Griffiths, etc.

Encore une remarque: alors même qu'on travaille avec des isomotifs, on peut associer à un tel $M$ quelque chose de mieux qu'une suite infinie de $\mathbb{Q}_{\ell^{-}}$ faisceaux (lorsqu'il y a une infinité de $\ell$ premiers aux car. résiduelles). En fait, on a ce qu'on pourrait appeler un faisceau "adélique", i.e. un faisceau de modules (moralement) sur l'anneau des adèles finis de $\mathbb{Q}$. De façon précise, on peut considérer tous les $T_{\ell}(M)$ sauf un nombre fini comme étant des $\mathbb{Z}_{\ell^{-}}$ faisceaux (pas seulement des $\mathbb{Q}_{\ell}$-faisceaux). Eliminant tout métaphysique motivique, on peut dire que la théorie de Jouanolou écrite en fixant un $\ell$, pourrait être développée avec des modifications techniques mineures pour avoir une théorie des " $A$-faisceaux", où $A$ est l'anneau des adèles, où un facteur direct $A^{\prime}$ de celui-ci obtenu en ne prenant qu'un paquet de nombres premiers (pas nécessairement tous). On obtient ainsi une théorie de coefficients (au sens technique dont nous avions discuté) ayant comme anneau de coefficients la $\mathbb{Q}$-algèbre $A$ resp. $A^{\prime}$. Comme $A$ et $A^{\prime}$ sont "absolutement plats", il n'y a pas introduction de $\underline{T o r}_{i}$ gênants et de canulars de degrés infinis dans cette théorie.

Pour en revenir au yoga des coefficients "discrets", où j'avais énoncé une propriété de trop apparemment, par contre il y en a une autre que nous n'avions pas explicitée. Il s'agit de la définition de l'objet de Tate sur $S$ comme l'inverse de l'objet (inversible pour $\otimes$ )

$$
T(-1)=R^{2} f_{*}\left(1_{P}\right)=R^{2} g_{!}\left(1_{E}\right)
$$

où $f: P \rightarrow S$ resp. $g: E \rightarrow S$ sont les projections de la droite projective 
resp. la droite affine sur S. D'autre part, ces objets (définis en fait sur le schéma de base $S_{0}$ de la théorie de coefficients) interviennent également dans la formulation des théorèmes de pureté relative ou absolus et la définition des classes fondamentales locales (qui, j'espère, doit être possible en termes des données initiales de la théorie de coefficients envisagée, sans constituer une donnée supplémentaire), et dans le calcul de $f^{!}$pour $f$ lisse (donc aussi pour $f$ lissifiable), pour ne parler que du démarrage du formalisme cohomologique. En fait, on les retrouve ensuite à chaque pas.

Une dernière remarque. Je crois qu'il vaudrait la peine de formaliser, dans le cadre d'une théorie de coefficients plus ou moins arbitraire, les arguments de dévissage qui ont conduit, dans le cas des coefficients étales, aux théorèmes de finitude pour $R f_{*}$ pour $f$ propre, puis pour $f$ séparé de type fini seulement (moyennant résolution des singularités). Ces dévissages apparaitraient maintenant comme des pas destinés à prouver l'existence de $R f_{*}$ (en même temps, s'il y a lieu, que sa commutation aux changements de base). A vrai dire, il n'est pas clair pour moi qu'on arrivera à des formulations qui s'appliqueraient directement aux $\mathbb{Z}_{\ell}$-faisceaux, disons; en fait, ce n'est pas ainsi que procède Jouanolou dans ce cas, qui au contraire se ramène aux énoncés déjà connus dans le cas des coefficients de torision (procédé qui n'a guère de chance de s'axiomatiser dans le contexte qui nous intéresse). Par contre, pensant directement au cas des motifs, on peut songer à utiliser un dévissage qui s'appuie entre autres sur les propriétés suivantes (quitte à se tirer par les lacets de souliers pour les établir chemin faisant): (a) un (iso)motif se dévisse en motifs sereins sur des schémas irréd. normaux (NB on suppose qu'on travaille sur des schémas excellents); (b) un motif serein sur un schéma normal irréductible se dévisse en motifs sereins "simples"-en fait, il suffit de faire le dévissage en le point générique; (c) un motif simple (pourvu qu'on remplace la base $S$ par un voisinage ouvert assez petit du point générique) est un facteur direct d'un $R^{i} f_{*}\left(1_{X}\right)$, où $f: X \rightarrow S$ est propre et lisse, tout du moins modulo tensorisation par un objet de Tate $T(j)$ convenable. Ainsi, moyennant au moins deux gros grains de sel qu'il faudrait essayer d'expliciter un jour, les motifs généraux (toujours iso, bien sûr) se ramènent aux motifs plus ou moins naïfs tels qu'ils sont décrits notamment dans Manin et Demazure. Cela s'applique tout au moins aux objets-quant aux morphismes, c'est une autre paire de manches-et encore pire pour les $\operatorname{Ext}^{i} \ldots$...

A ce propos, on peut se convaincre que l'application qui va des classes d'extension de deux motifs (dans la catégorie abélienne des motifs) vers le Ext $^{1}$ défini comme $\operatorname{Hom}(M, N[1])$ (Hom dans la catégorie triangulée) ne devrait pas être bijective (mais sans doute injective). Plaçons-nous en effet sur une base $S$ spectre d'un corps fini, prenons pour $M$ et $N$ le motif

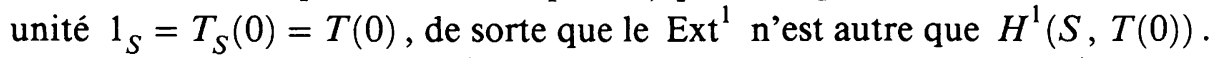
Les calculs $\ell$-adiques du $H^{1}$ nous suggèrent fortement que le $H^{1}$ absolu 
motivique est canoniquement isomorphe à $\mathbb{Q}$. Mais d'autre part les classes d'extension de $T(0)$ par $T(0)$ doivent être nulles (plus généralement, les extensions de $M$ par $N$ doivent être nulles (sur tout corps $K$ ) si $M$ et $N$ sont des motifs de poids $r$ et $s$ avec $r \leq s$, si on admet le yoga de la filtration d'un motif par poids croissants, avec gradué associé semi-simple. (NB En fait, sur un corps fini, la catégorie des motifs devrait être toute entière semi-simple, i.e. toute extension devrait être triviale, i.e. la filtration croissante précédente devrait splitter canoniquement: cela résulte du fait que l'endomorphisme de frobenius du motif opère avec des "poids" différents sur les composants des différents poids-plus un petit exercice de catégories tannakiennes.)

Bien cordialement

Alexandre

\section{REFERENCES}

[Beau] A. Beauville, Sur l'anneau de Chow d'une variété abélienne, Math. Ann. 273 (1986), 647-651.

[Be1] A. Beilinson, Higher regulators and values of L-functions, J. Soviet Math. 30 (1985), 2036-2070.

[Be2] - Notes on absolute Hodge cohomology, Applications of Algebraic $K$-Theory to Algebraic Geometry and Number Theory. I, Contemp. Math., vol. 55, Amer. Math. Soc., Providence, RI, 1986, pp. 35-68.

[Be3] - Height pairing between algebraic cycles, $K$-Theory, Arithmetic and Geometry (Y. Manin, ed.), Lecture Notes in Math., vol. 1289, Springer-Verlag, New York, 1987, pp. 1-26.

[Be4] On the derived category of perverse sheaves, $K$-Theory, Arithmetic and Geometry (Y. Manin ed.), Lecture Notes in Math., vol. 1289, Springer-Verlag, New York, 1987, pp. $27-41$.

[BBD] A. Beilinson, J. Bernstein, and P. Deligne, Faisceaux pervers, Analyse et Topologie sur les Espaces Singulières I, Astérisque 100 (1982).

[B11] S. Bloch, An example in the theory of algebraic cycles, Algebraic $K$-Theory, Evanston, 1976 (M. Stein, ed.), Lecture Notes in Math., vol. 551, Springer-Verlag, New York, 1976, pp. 1-29.

[B12] _ Lectures on Algebraic Cycles, Duke Univ. Math. Ser., vol. IV, Duke Univ., Durham, NC, 1980.

[BK] S. Bloch and K. Kato, L-functions and Tamagawa numbers of motives, Grothendieck Festschrift, vol. 1, Progr. Math., vol. 86, Birkhäuser, Boston, MA, 1990, pp. 333-400.

[BO] S. Bloch and A. Ogus, Gersten's conjecture and the homology of schemes, Ann. Sci. École Norm. Sup. (4) 7 (1979), 181-202.

[BS] S. Bloch and V. Srinivas, Remarks on correspondences and algebraic cycles, Amer. J. Math. 105 (1983), 1235-1253.

[Ca] J. Carlson, Extension of mixed Hodge structures, Journées de Géométrie Algébrique d'Angers 1979, Sijthoff \& Noordhoff, Alphen aan den Rijn, 1980, pp. 107-127.

[CTRS] J.-L. Colliot-Thélène, W. Raskind, and S. Saito, Nonrepresentability of Chow groups of 0 -cycles and other $K$-cohomology groups, preprint.

[CTSS] J.-L. Colliot-Thélène, J.-J. Sansuc, and C. Soulé, Torsion dans le groupe de Chow de codimension deux, Duke Math. J. 50 (1983), 763-801.

[De1] P. Deligne, Théorème de Lefschetz et critères de dégénérescence de suites spectrales, Inst. Hautes Études Sci. Publ. Math. 35 (1968), 107-126.

[De2] — Théorie de Hodge. II, Inst. Hautes Études Sci. Publ. Math. 40 (1972), 5-57.

[De3] $\longrightarrow$ La conjecture de Weil. II, Inst. Hautes Études Sci. Publ. Math. 52 (1981), 313-428.

[De4] _ A quoi servent les motifs?, these Proceedings, vol. 1, pp. 143-161. 
[De5] _ Décompositions dans la catégorie dérivée, these Proceedings, vol. 1, pp. 115-128.

[DM] P. Deligne and J. Milne, Tannakian categories, Hodge Cycles, Motives, and Shimura Varieties, Lecture Notes in Math., vol. 900, Springer-Verlag, New York, 1982, pp. 101-208.

[Dem] M. Demazure, Motifs des variétés algébriques, Sém. Bourbaki 1969-1970, exposé 365, Lecture Notes in Math., vol. 180, Springer-Verlag, New York, 1971.

[DV] A. Douady, J.-L. Verdier, et al., Séminaire de géométrie analytique, Astérisque 36-37, 1976.

[Ek] T. Ekedahl, On the adic formalism, Grothendieck Festschrift, vol. 2, Progr. Math., vol. 86, Birkhäuser, Boston, MA, 1990, pp. 197-218.

[EL] H. Esnault and M. Levine, On the injectivity and surjectivity of cycle maps, preprint 1992.

[EV] H. Esnault and E. Viehweg, Deligne-Beilinson cohomology, Beilinson's Conjectures on Special Values of L-Functions (Rapoport, Schappacher, and Schneider, eds.), Perspect. Math., vol. 4, Academic Press, New York, 1988, pp. 43-91.

[Fa] C. Faber, Chow rings of moduli spaces of curves. I: The Chow ring of $\overline{\mathscr{M}}_{3}$, Ann. of Math. (2) 132 (1990), 331-419; II: Some results on the Chow ring of $\overline{\mathscr{M}}_{4}$, Ann. of Math. (2) 132 (1990), 421-449.

[FPR] J.-M. Fontaine and B. Perrin-Riou, Autour des conjectures de Bloch et Kato: Cohomologie galoisienne et valeurs de fonctions $L$, these Proceedings, vol. 1, pp. 599-706.

[Fu] W. Fulton, Intersection Theory, Springer-Verlag, New York, 1984.

[Gi] H. Gillet, Riemann-Roch theorems for higher algebraic K-theory, Adv. Math. 40 (1981), 203-289.

[Gray] D. Grayson, Weight filtrations in algebraic K-theory, these Proceedings, vol. 1, pp. 207-237.

[Gr1] A. Grothendieck, Technique de descente et théorèmes d'existence en géométrie algébrique (TDTE). V. Les schémas de Picard. Théorèmes d'existence, Sém. Bourbaki 14 (1961/ 62), exp. 232.

[Gr2] _ TDTE. VI. Les schémas de Picard. Propriétés générales, Sém. Bourbaki 14 (1961/ 62), exp. 236.

[Gr3] _ Le groupe de Brauer. III: Exernples et complements, Dix exposés sur la cohomologie des schémas, North-Holland, Amsterdam, 1968, pp. 88-108.

[Gr4] Hodge's general conjecture is false for trivial reasons, Topology 5 (1969), 299-303.

[Ja1] U. Jannsen, Continuous étale cohomology, Math. Ann. 280 (1988), 207-245.

[Ja2] Deligne homology, Hodge-D-conjecture, and motives, Beilinson's Conjectures on Special Values of L-Functions (M. Rapoport, N. Schappacher, and P. Schneider, eds.), Perspect. Math., vol. 4, Academic Press, New York, 1988, pp. 305-372.

[Ja3] - Mixed Motives and Algebraic K-Theory, Lecture Notes in Math., vol. 1400, Springer-Verlag, New York, 1990.

[KS] K. Kato and S. Saito, Unramified class field theory for arithmetical surfaces, Ann. of Math. (2) 118 (1983), 241-275.

[KM] N. Katz and W. Messing, Some consequences of the Riemann hypothesis for varieties over finite fields, Invent. Math. 23 (1974), 73-77.

[K11] S. Kleiman, Algebraic cyles and the Weil conjectures, Dix exposés sur la cohomologie des schémas, North-Holland, Amsterdam, 1968, pp. 359-386.

[Kl2] _ Motives, Algebraic Geometry, Oslo 1970 (F. Oort, ed.), Wolters-Noordhoff, Groningen, 1972, pp. 53-82.

[K13] S. Kleiman, The standard conjectures, these Proceedings, vol. 1, pp. 3-20.

[Kü1] K. Künnemann, On the Chow motive of an abelian scheme, these Proceedings, vol. 1, pp. 189-205.

[Kü2] _ A Lefschetz decomposition for Chow motives of abelian schemes, preprint (1992).

[Le] J. Lewis, Towards a generalization of Mumford's theorem, J. Math. Kyoto Univ. 29 (1989), 195-204.

[Li] S. Lichtenbaum, Motivic complexes, these Proceedings, vol. 1, pp. 303-313.

[Lie] D. Lieberman, Intermediate Jacobians, Algebraic Geometry, Oslo 1970 (F. Oort, ed.), Wolters-Noordhoff, Groningen, 1972, pp. 125-139. 
[Ma] Y. Manin, Correspondences, motives, and monoidal transformations, Mat. Sb. 77 (1968) = Math. USSR-Sb. 6 (1968), 439-470.

[MS] N. Maruyama and N. Suwa, Remarques sur un article de Bloch et Srinivas sur les cycles algébriques, J. Fac. Sci. Univ. Tokyo 35 (1988), 371-380.

[Mi] J. Milne, Étale Cohomology, Princeton Math. Ser., vol. 33, Princeton Univ. Press, Princeton, NJ, 1980.

[Mo] S. Mori, On Tate conjecture concerning endomorphisms of abelian varieties, Proceedings of the Internat. Sympos. of Alg. Geometry, Kyoto 1977 (M. Nagata, ed.), Kinokuniya Book-Store Co., Tokyo, pp. 219-230.

[Mum1] D. Mumford, Rational equivalence of 0-cycles on surfaces, J. Math. Kyoto Univ. 9 (1969), 195-204.

[Mum2] _ Abelian Varieties, Tata Inst. Fund. Res. Stud. Math., vol. 5, Oxford Univ. Press, London, 1970/1974/1985.

[Mum3] _ Towards an enumerative geometry of the moduli space of curves, Arithmetic and Geometry (M. Artin and J. Tate, eds.), Birkhäuser, Boston, Basel, and Stuttgart, 1983, pp. 271-328.

[Mur1] J. P. Murre, On the motive of an algebraic surface, J. Reine Angew. Math. 409 (1990), 190-204.

[Mur2] _ On a conjectural filtration on the Chow groups of an algebraic variety (parts I and II), Indag. Math. New Series Vol. 4 (1993), 177-201.

[Nek] J. Nekovár, Beilinson's conjectures, these Proceedings, vol. 1, pp. 537-570.

[Pa] K. Paranjape, Cohomological and cycle theoretic connectivity, preprint (1992).

[Roi1] A. Roitman, Rational equivalence of zero-cycles, Mat. Sb. 89 (1972) = Math. USSR-Sb. 18 (1974), 571-588.

[Roi2] , The torsion in the group of zero-cycles modulo rational equivalence, Ann. of Math. (2) 111 (1980), 553-569.

[SaM1] M. Saito, Mixed Hodge modules, Publ. Res. Inst. Math. Sci. 26 (1990), 221-333.

[SaM2] _ On the formalism of mixed sheaves, preprint RIMS Kyoto Univ. (1991).

[SaS] S. Saito, Motives and filtrations on Chow groups, preprint (1992).

[Schoe] C. Schoen, On Hodge structures and non-representability of Chow groups, preprint (1991).

[Scho] A. Scholl, Classical motives, these Proceedings, vol. 1, pp. 163-187.

[Sou1] C. Soulé, Groupes de Chow et $K$-théorie de variétés sur un corps fini, Math. Ann. 268 (1984), 317-345.

[Sou2] _ Opérations en K-théorie algébrique, Canad. J. Math. 37 (1985), 488-550.

[St] J. Steenbrink, A summary of mixed Hodge theory, these Proceedings, vol. 1, pp. 31-41.

[Ta] J. Tate, Conjectures on algebraic cycles in $\ell$-adic cohomology, these Proceedings, vol. 1, pp. $71-83$.

[Ver] J.-L. Verdier, Catégories dérivées (Etat 0), SGA $4 \frac{1}{2}$ (Cohomologie Étale), Lecture Notes in Math., vol. 569, Springer-Verlag, New York, 1977, pp. 262-311.

[We] A. Weil, Sur les critères d'équivalence en géométrie algébrique, Math. Ann. 128 (1954), 95-127.

[Za] J. Zarhin, Abelian varieties in characteristic $p$, Mat. Zametki $19(1976)=$ Math. Notes 19 (1976), 240-244.

Mathematisches Institut, Universität ZU Köln, Weyertal 86-90, 50931 Köln, GerMANY 